\title{
Metrological Tools for the Reference Materials and Reference Instruments of the NIST Material Measurement Laboratory
}

Carlos R. Beauchamp, Johanna E. Camara, Jennifer Carney, Steven J. Choquette,

Kenneth D. Cole, Paul C. DeRose, David L. Duewer, Michael S. Epstein, Margaret C. Kline, Katrice A. Lippa, Enrico Lucon, John Molloy, Michael A. Nelson, Karen W. Phinney, Maria Polakoski, Antonio Possolo, Lane C. Sander, John E. Schiel, Katherine E. Sharpless, Blaza Toman, Michael R. Winchester, Donald Windover

This publication is available free of charge from: https://doi.org/10.6028/NIST.SP.260-136-2021
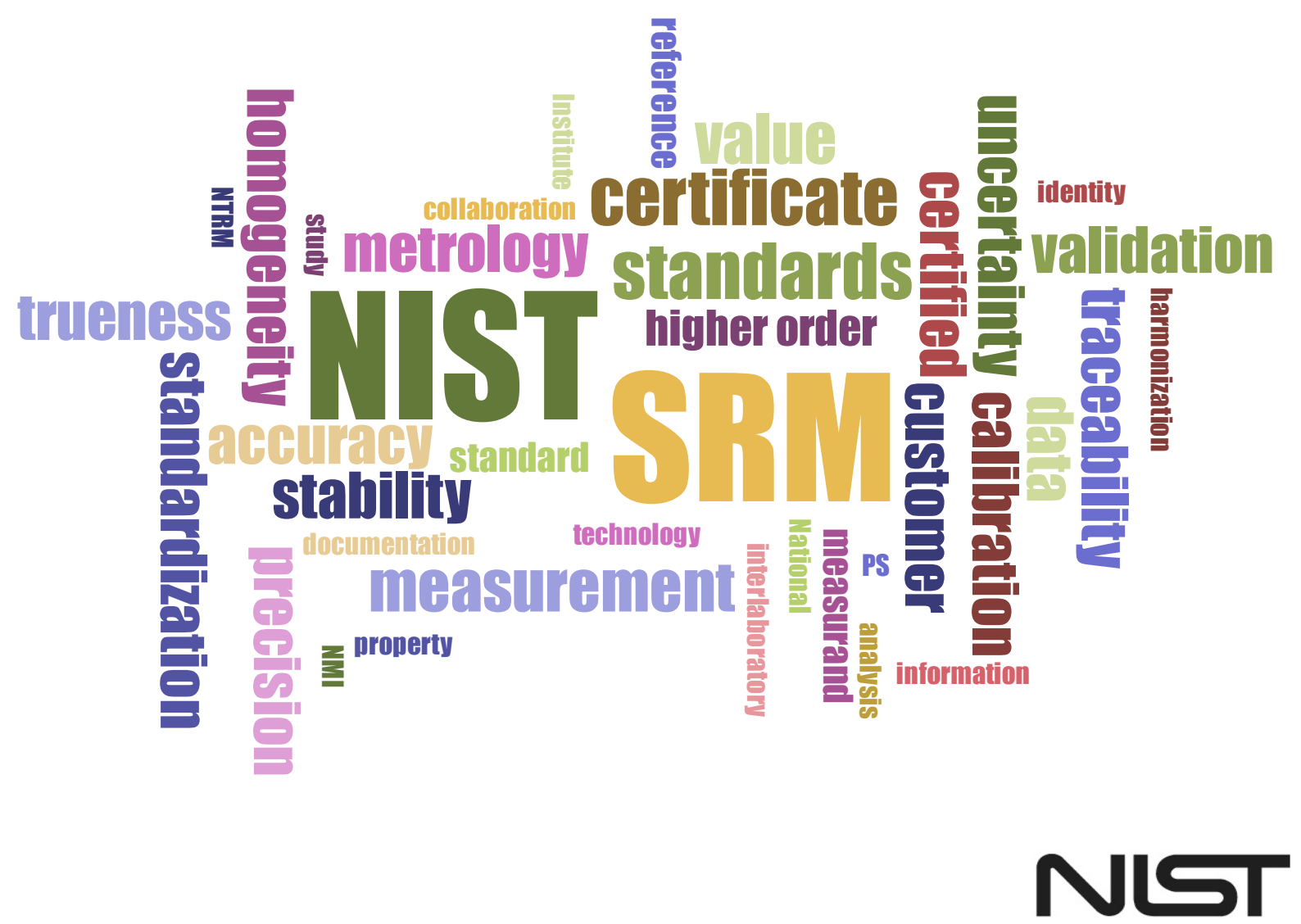

National Institute of Standards and Technology U.S. Department of Commerce 
This page intentionally blank. 


\title{
Metrological Tools for the Reference Materials and Reference Instruments of the NIST Material Measurement Laboratory
}

\author{
Enrico Lucon \\ Applied Chemicals and Materials Division \\ Margaret C. Kline, Karen W. Phinney, John E. Schiel \\ Biomolecular Measurement Division \\ Kenneth D. Cole, Paul C. DeRose \\ Biosystems and Biomaterials Division
}

Johanna E. Camara, Jennifer Carney, David L. Duewer, Michael S. Epstein, Lane C. Sander, Katrice A. Lippa, Michael A. Nelson, John Molloy, Michael R. Winchester Chemical Sciences Division

Donald Windover

Materials Measurement Science Division

Carlos R. Beauchamp

Materials Science and Engineering Division

Steven J. Choquette, Maria Polakoski

Office of Reference Materials

Antonio Possolo, Blaza Toman

Statistical Engineering Division

Katherine E. Sharpless

Special Programs Office

This publication is available free of charge from:

https://doi.org/10.6028/NIST.SP.260-136-2021

September 2021

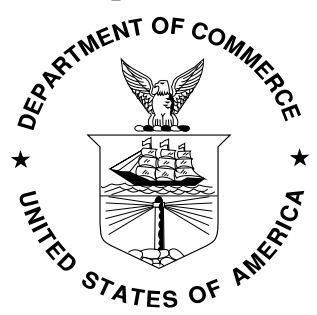

U.S. Department of Commerce Gina M. Raimondo, Secretary

National Institute of Standards and Technology James K. Olthoff, Performing the Non-Exclusive Functions and Duties of the Under Secretary of Commerce for Standards and Technology \& Director, National Institute of Standards and Technology 
Certain commercial entities, equipment, or materials may be identified in this document to describe an experimental procedure or concept adequately. Such identification is not intended to imply recommendation or endorsement by the National Institute of Standards and Technology, nor is it intended to imply that the entities, materials, or equipment are necessarily the best available for the purpose.

National Institute of Standards and Technology Special Publication 260-136-2021 Natl. Inst. Stand. Technol. Spec. Publ. 260-136-2021, 66 pages (September 2021) CODEN: NSPUE2

This publication is available free of charge from: https://doi.org/10.6028/NIST.SP.260-136-2021 


\section{Foreword}

The National Institute of Standards and Technology (NIST), formerly the National Bureau of Standards, was established by the U.S. Congress in 1901 and charged with establishing a measurement foundation to facilitate U.S. and international commerce. NIST provides reference materials and reference instruments that are used to ensure the accuracy, metrological traceability, and comparability of measurement results in many diverse fields of science, industry, and technology. This document provides and explains the vocabulary used at NIST to describe the reference materials and reference instruments available to NIST's customers. This document also describes the methods used in developing NIST reference materials. The definitions and descriptions provided supersede those used in the original versions of this publication, NIST SP260-136 ${ }^{1}$ and SP260-136-2020 ${ }^{2}$.

\section{Scope}

The NIST Material Measurement Laboratory (MML) serves as the U.S. national reference laboratory for measurements in the chemical, biological, and material sciences. MML activities include the development and dissemination of reference materials and reference instruments to assure the quality of measurement results. While other NIST organizational units develop reference materials and reference instruments, MML produces the majority of NIST reference materials and, via its Office of Reference Materials, supports the production and dissemination of all of NIST's reference materials and instruments. The definitions and descriptions provided in this document apply to NIST's certified reference materials (CRMs) and reference instruments issued after the publication of this document. 


\section{CONTENTS}

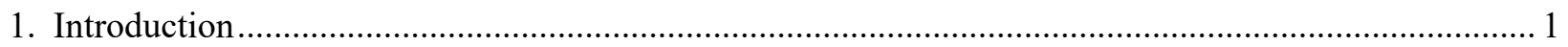

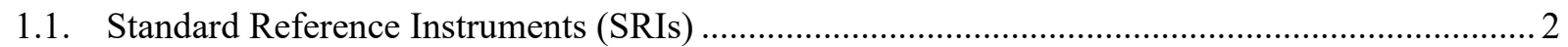

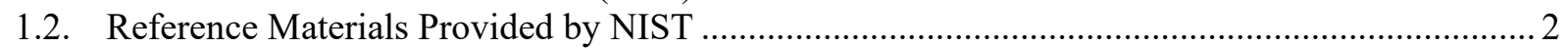

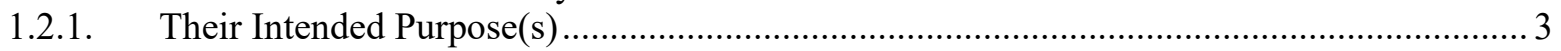

1.2.2. Certified Versus Non-Certified Values......................................................................... 4

1.2.3. Reference, Information, and Indicative Values........................................................... 4

1.2.4. Documenting Certified Values........................................................................ 4

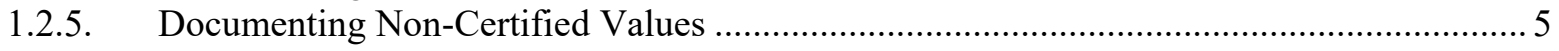

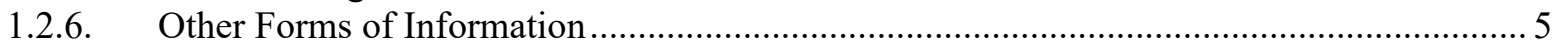

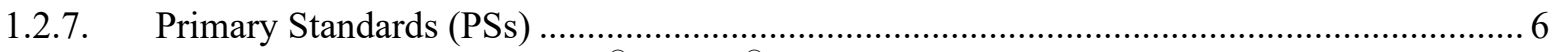

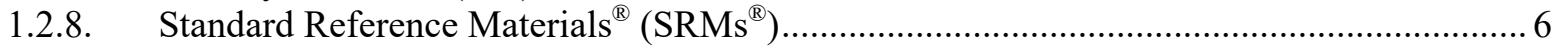

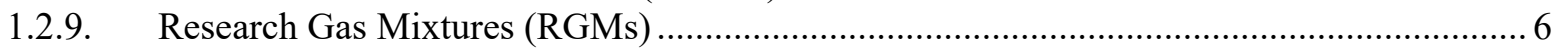

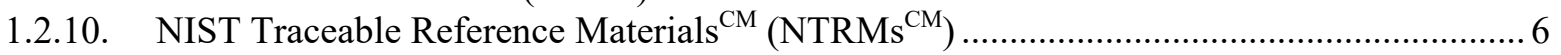

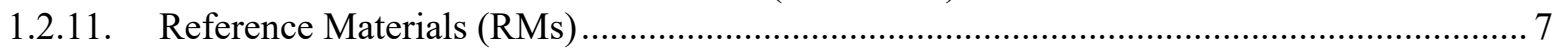

1.2.12. Research-Grade Test Materials (RGTMs) and Other Exploratory Materials ..................... 7

1.3. Stages in the Reference Material Project Development Process .................................................. 8

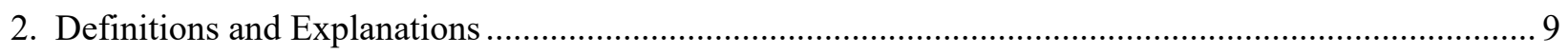

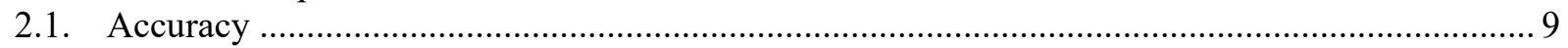

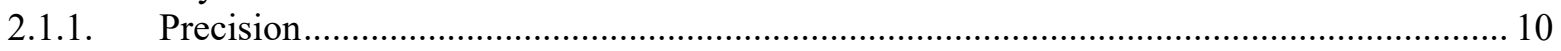

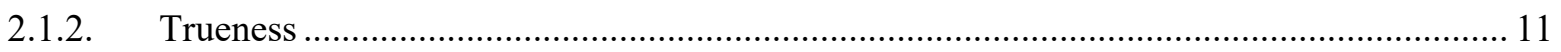

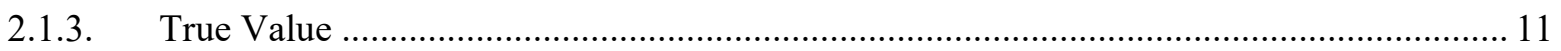

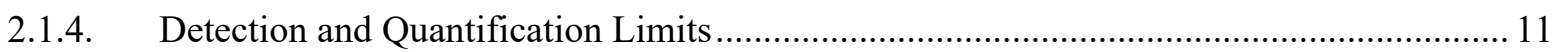

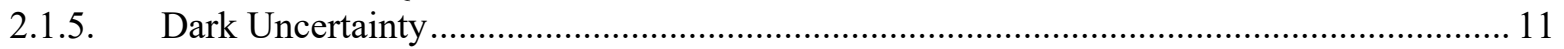

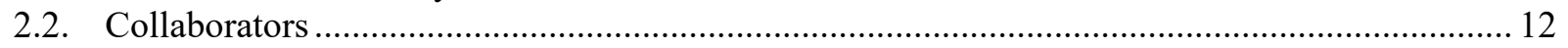

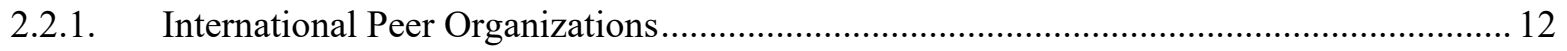

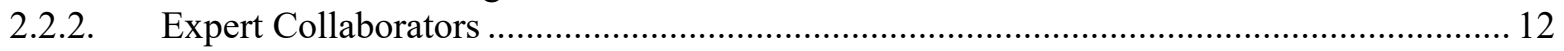

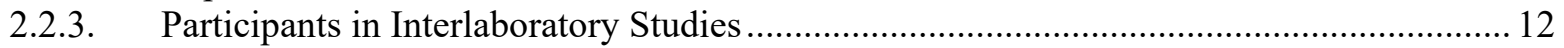

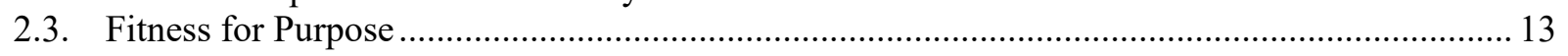

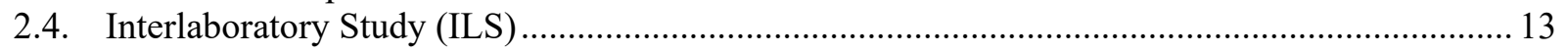

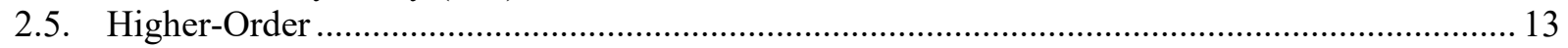

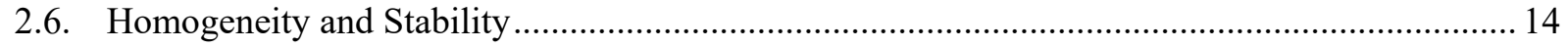

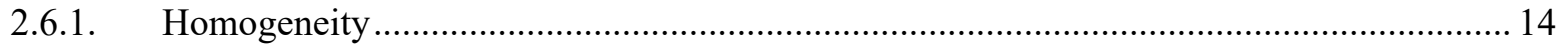

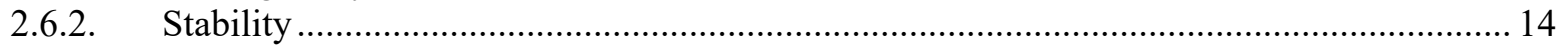

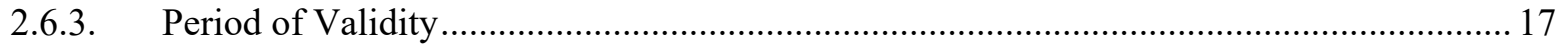

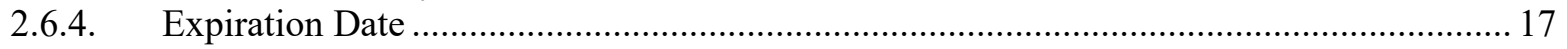

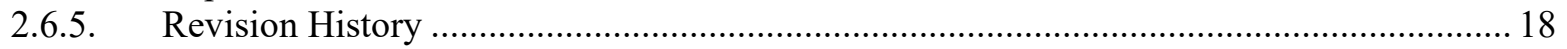

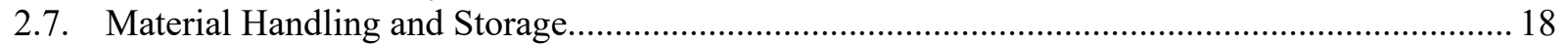

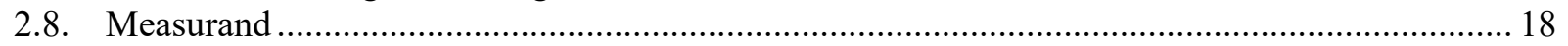

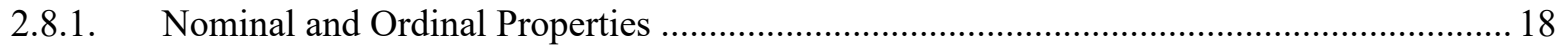

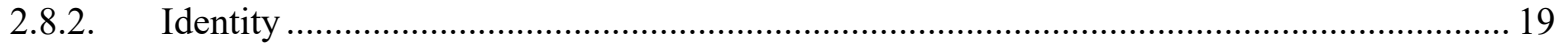

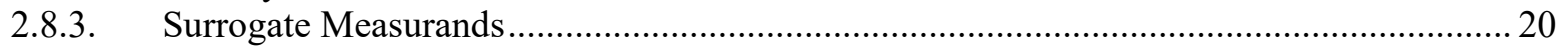

2.9. Measurement Method, Procedure, and Process ........................................................................... 20

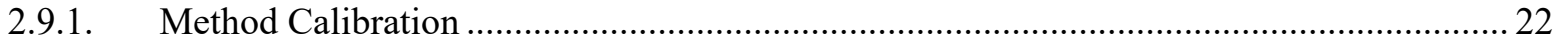

2.9.2. Method/Procedure/Process Validation (and Verification) ................................................ 23

2.9.3. Method/Procedure/Process Independence ...................................................................... 24

2.9.4. Reference Measurement Procedure (RMP) ...................................................................... 25

2.9.5. Primary Reference Measurement Procedure ("Primary Method") ...................................2 25

2.9.6. Higher-Order Reference Measurement Procedure ....................................................... 26

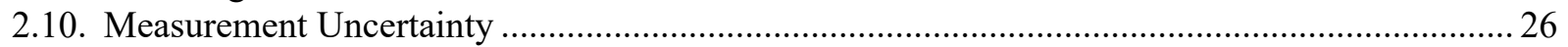




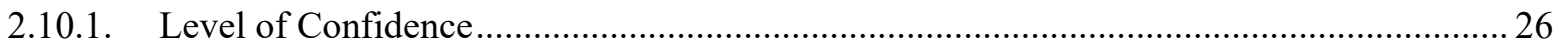

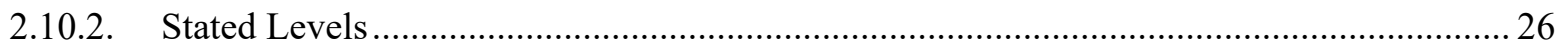

2.10.3. Uncertainty for Non-Certified Values...................................................................... 26

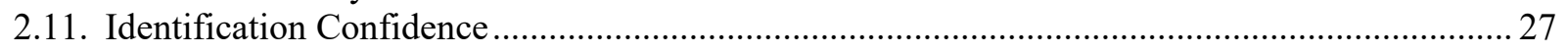

2.11.1. Confidence for Non-Certified Identities ..................................................................... 27

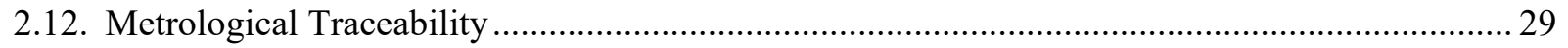

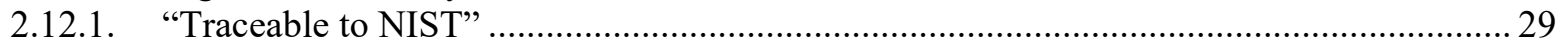

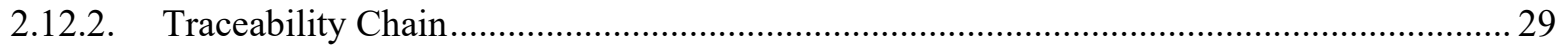

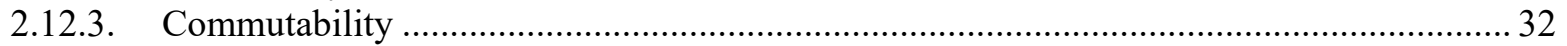

2.12.4. Standardization and Harmonization................................................................................. 34

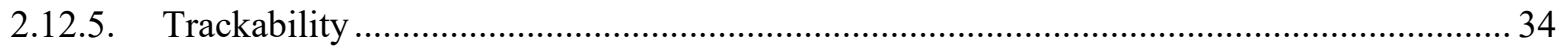

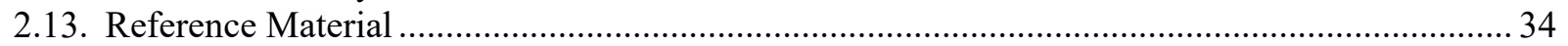

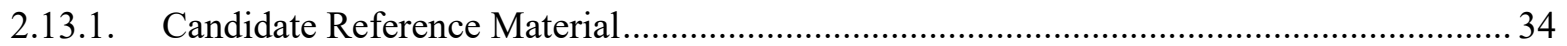

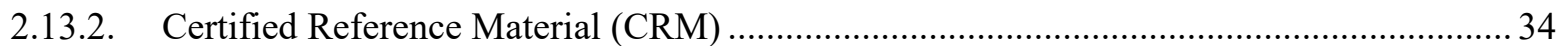

2.13.3. Matrix Reference Material .................................................................................... 35

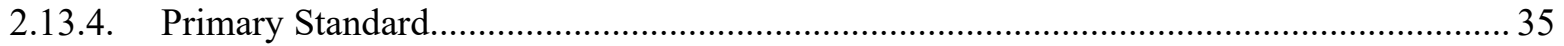

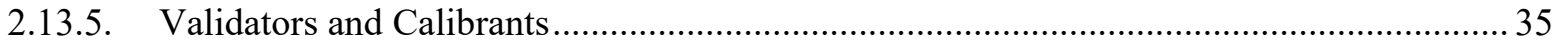

2.14. Secondary Standard Producer ............................................................................................ 36

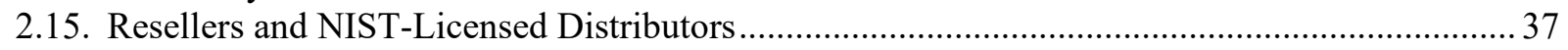

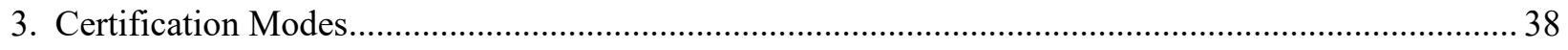

3.1. One Higher-Order Reference Measurement Procedure at NIST ..................................................38

3.2. Two or More Independent Reference Measurement Procedures at NIST ..................................... 40

3.3. One Reference Measurement Procedure at NIST and Expert Collaborators or Interlaboratory

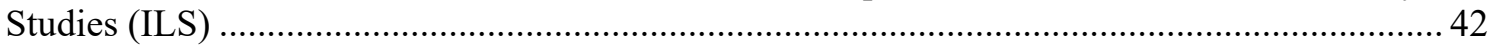

3.4. Value Transfer from an Existing Standard Reference Material or Primary Standard.................. 43

3.5. Operationally Defined Measurands....…………………....................................................... 43

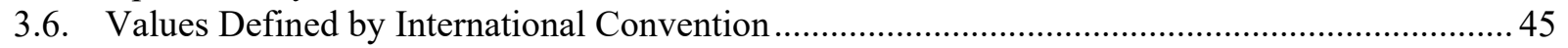

3.7. Need-Driven Exceptions .................................................................................................... 45

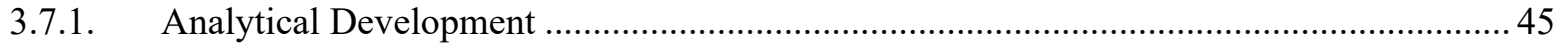

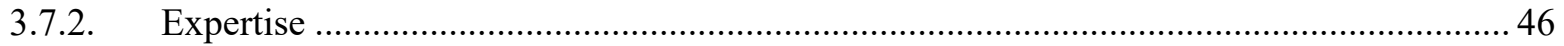

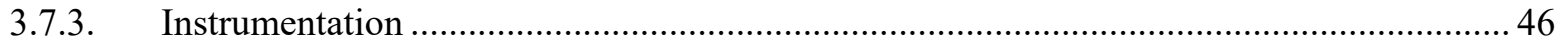

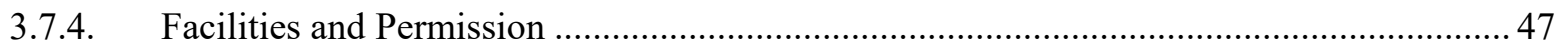

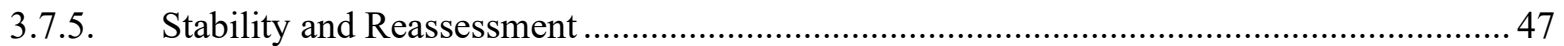

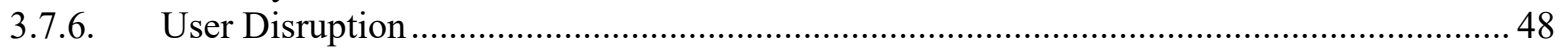

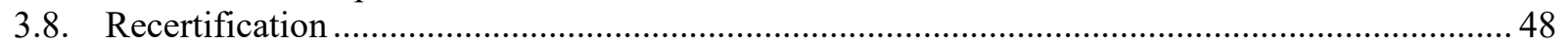

3.9. Non-Certified Values ....................................................................................................... 48

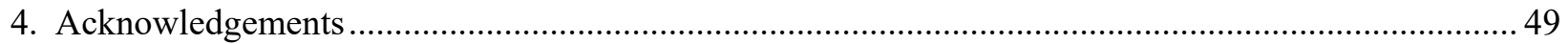

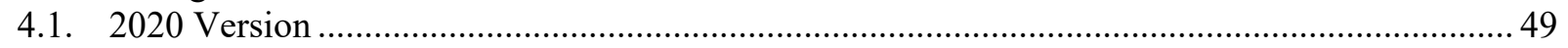

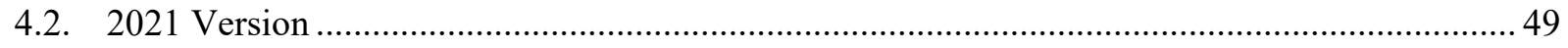

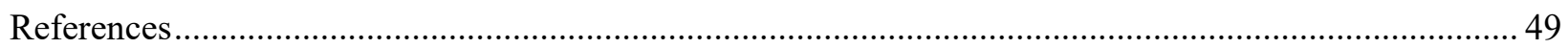

\section{FIGURES}

Figure 1. Traceability Hierarchy Among NIST's Measurement Tools ............................................... 1

Figure 2. Relationships Among NIST's Reference Materials ........................................................... 3

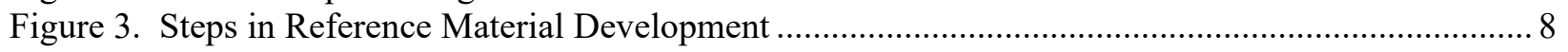

Figure 4. Relationships Between Measurement Accuracy, Precision, and Trueness ............................. 10

Figure 5. Probability Densities for Precision Conditions at Fixed Trueness ........................................... 11

Figure 6. Stability of Selected Elements in SRM 1566b Oyster Tissue ................................................ 16 
Figure 7. Stability of Total $\beta$-Carotene in Frozen Human Serum .......................................................... 17

Figure 8. Steps in a Generic Measurement Process for Certifying Values ............................................. 21

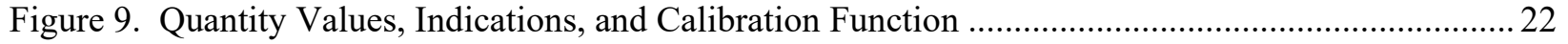

Figure 10. Quantity Values, Indications, and Analysis Function .........................................................2

Figure 11. Process for Establishing the Confidence in an Assigned Identity ........................................28

Figure 12. Metrological Traceability of Results from a NIST Standard Reference Instrument (SRI). ..... 30

Figure 13. Metrological Traceability for Results Using NIST's Reference Materials ............................ 31

Figure 14. Metrological Traceability for Results When a Matrix Material is Used as a Calibrant ........... 32

Figure 15. Commutability Responsibilities for Producers and Users of Matrix CRMs ...........................33

\section{ABBREVIATIONS AND ACRONYMS}

$\begin{array}{ll}\text { BIPM } & \text { International Bureau of Weights and Measures } \\ \text { CCQM } & \text { Consultative Committee for Amount of Substance: Metrology in Chemistry and } \\ \text { CIPM } & \text { Biology } \\ \text { International Committee for Weights and Measures } \\ \text { DRM } & \text { certified reference material } \\ \text { designated institute } \\ \text { EPA } & \text { US Environmental Protection Agency } \\ \text { GUM } & \text { Guide to the Expression of Uncertainty in Measurement } \\ \text { ICP-OES } & \text { inductively coupled plasma optical emission spectroscopy } \\ \text { ILS } & \text { interlaboratory study } \\ \text { ID-LC-MS/MS } & \text { isotope dilution liquid chromatography tandem mass spectrometry } \\ \text { ID-MS } & \text { isotope dilution mass spectrometry } \\ \text { JCTLM } & \text { Joint Committee for Traceability in Laboratory Medicine } \\ \text { NIST } & \text { National Institute of Standards and Technology } \\ \text { NMI } & \text { national metrology institute } \\ \text { NTRM } & \text { NIST Traceable Reference Material } \\ \text { ORM } & \text { Office of Reference Materials } \\ \text { PCR } & \text { polymerase chain reaction } \\ \text { PS } & \text { primary standard } \\ \text { qNMR } & \text { quantitative nuclear magnetic resonance } \\ \text { RGM } & \text { Research Gas Mixture } \\ \text { RGTM } & \text { Research-Grade Test Material } \\ \text { RM } & \text { Reference Material (produced by NIST) } \\ \text { RMP } & \text { reference measurement procedure } \\ \text { SI } & \text { International System of Units } \\ \text { SRI } & \text { Standard Reference Instrument } \\ \text { SRM } & \text { Standard Reference Material } \\ \text { VIM } & \text { International Vocabulary of Metrology } \\ \text { XRF } & \text { X-ray fluorescence spectrometry } \\ 24 R, 25(O H){ }_{2} D_{3} & \text { 24R,25-dihydroxyvitamin D } \\ 25(O H) D_{2} & \text { 25-hydroxyvitamin D } \\ & \end{array}$




\section{Introduction}

NIST's mission is to promote U.S. innovation and industrial competitiveness by advancing measurement science, standards, and technology in ways that enhance economic security and improve quality of life. One way in which NIST fulfills this mission is through the delivery of reference materials and reference instruments. NIST develops, documents, and delivers these reference materials and reference instruments in accordance with international standards and guidelines ${ }^{3,4,5,6}$ as described in NIST's Quality Management System for Measurement Services. ${ }^{7,8}$ Techniques and methods used at NIST for characterizing measurement procedures and evaluating the chemical and physical properties of materials are critically examined. Potential sources of bias and variability in these processes are addressed. ${ }^{9,10}$ Details of NIST's processes are documented in publicly accessible NIST Technical Series publications or in refereed scientific and technical journals.

This publication describes the reference materials and reference instruments provided by NIST's Material Measurement Laboratory (MML). Figure 1 displays the metrological validity and traceability hierarchy of these tools; note that links are provided for terms used throughout this document. See Section 1.2 for in-depth discussions of the reference material tools, Section 0 for definitions of terms, and Section 3 for descriptions of how property values are assigned to these tools.

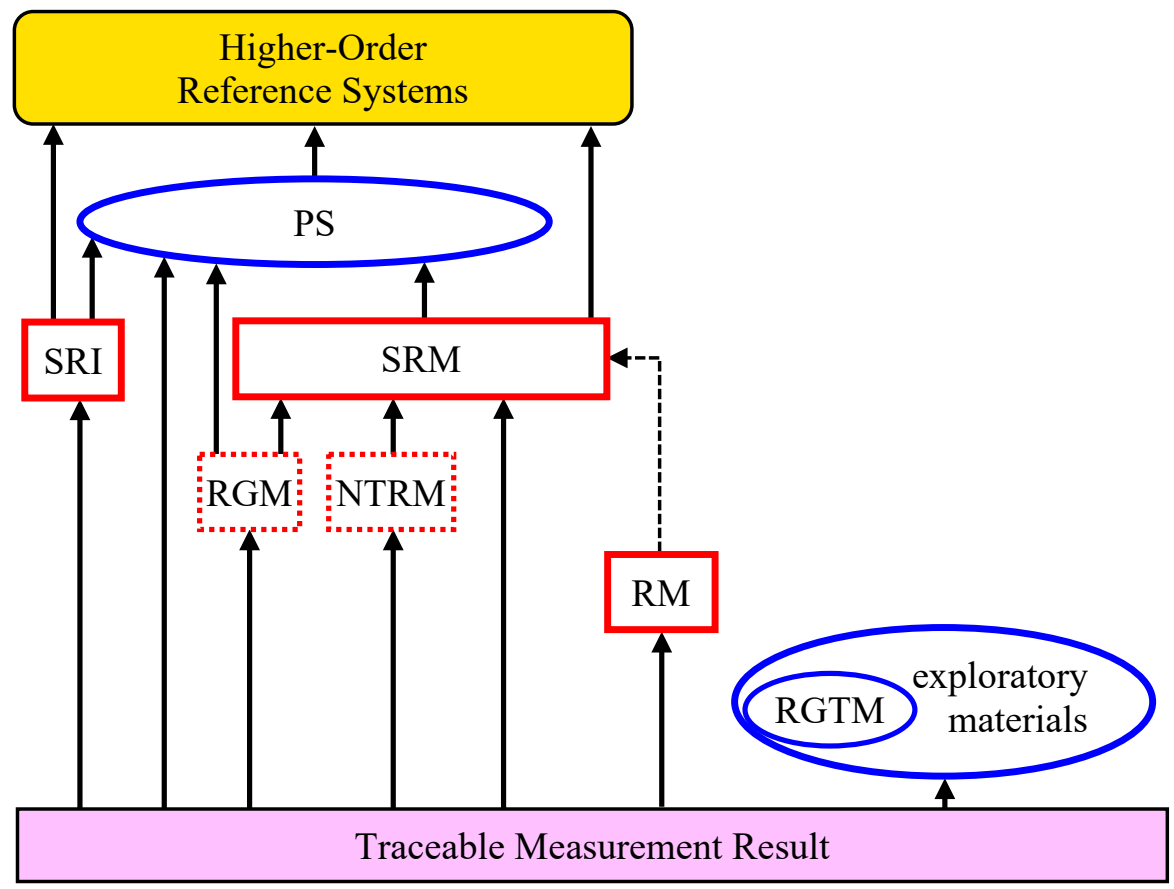

Figure 1. Traceability Hierarchy Among NIST's Measurement Tools

The Standard Reference Instrument (SRI), Standard Reference Material (SRM), and NIST's Reference Material (RM) measurement tools (solid red rectangles) are available for customer purchase. The Research Gas Mixture (RGM) and NIST Traceable Reference Material (NTRM) tools (dotted red rectangles) are produced in collaboration with industrial partners and are used by them to provide services to their customers. The primary standard (PS), Research-Grade Test Material (RGTM), and other exploratory materials (blue ovals) are used primarily by NIST to support its own measurement procedures. Arrows indicate linkages among and between tools and the traceable measurement results. Metrological traceability linkages and confidence in the metrological validity of the assigned values improve from bottom to top. The dotted arrow indicates that the assigned values for RMs may or may not be traceable to SRM values. Results linked to RMs, RGTMs, and other exploratory materials are suitable for between-method harmonization, within-method precision, withinmethod stability assessment, and interlaboratory studies. Results linked to SRIs and PSs are, in addition, suitable for method calibration and validation. Experience gained from RGTMs and other exploratory materials is intended to contribute to the development of higher-order reference materials. 


\subsection{Standard Reference Instruments (SRIs)}

Standard Reference Instruments (SRIs) are devices that have been designed and validated by NIST and are then sold to qualified customers. SRIs transfer to these customers the ability to obtain traceable results when metrological traceability cannot be adequately established through reference materials. Some SRIs allow customers to directly realize an International System of Units (SI) unit (i.e., to experimentally establish the value consistent with the SI definition of the unit). SRIs are issued as "SRI 6xxx" where " $\mathrm{xxx}$ " is a unique series of three digits (examples given below).

As shown in Figure 1, measurement results produced using an SRI can be directly linked to a higher-order reference system. Such direct metrological traceability minimizes the uncertainty in measurement results. While ideally all measurements could be made using SRIs, an SRI is economically feasible in practice only when artifact reference materials or existing calibration services cannot provide fit-for-purpose calibration of less-specialized measurement devices. Most SRIs are used for physical measurements requiring extreme accuracy or for measurands that are not amenable to being captured in a container. MML currently provides the following SRIs:

- SRI 6005a and 6005b Polymerization Stress Tensometers ${ }^{11,12}$

A polymerization stress tensometer (PST) is a cantilever-beam based measurement instrument that combines beam deflection mechanics with capacitive sensor technology to measure real-time polymerization shrinkage stress during photocuring of composite resins. Measurement results from a PST can be made traceable to the SI through calibration services offered by NIST.

- SRI 6008 Ozone Standard Reference Photometer (SRP) ${ }^{13,14}$

The NIST Standard Reference Photometer accurately measures concentrations of ozone $\left(\mathrm{O}_{3}\right)$ in air. The measurement principle is based on the Beer-Lambert Law using an internationally accepted absorption cross-section. The SRP is a dual-cell instrument in which the charging of each cell alternates between sample and reference sample streams. Ozone concentrations obtained using new SRPs typically agree with those from SRP 2 (the master SRP maintained at NIST) to better than $0.3 \%$. The SRP is used by the International Committee for Weights and Measures (CIPM)'s International Bureau of Weights and Measures (BIPM) as the primary standard for numerous national and international ozone-monitoring networks. SRP 0 (a second NIST SRP) is directly compared to the BIPM's SRP every two years. SRP 0 is then used to transfer the comparison to SRP 2.

\subsection{Reference Materials Provided by NIST}

NIST currently provides six named types of materials intended to be used as measurement standards: primary standards (PSs), Standard Reference Materials (SRMs), Research Gas Mixtures (RGMs), NIST Traceable Reference Materials (NTRMs), Reference Materials (RMs), and Research-Grade Test Materials (RGTMs). These standards are all "reference materials" under the BIPM's International Vocabulary of Metrology (VIM) definition: materials, sufficiently homogeneous and stable with respect to one or more specified properties, which have been determined by NIST to be fit for their intended use [wording adapted from VIM 5.13]. The RGTMs are a subset of a broader class of "exploratory" materials that NIST uses when investigating new measurement challenges. Not all exploratory materials are intended to be used as - or indeed suited for development to become - any other type of reference material that NIST provides.

Although the acronym "RM" is commonly used for the generic concept "reference material," in this document the acronym "RM" is used only in the context of NIST-provided reference materials. The expanded term "reference material" is used when the generic concept is discussed.

A subset (PSs, SRMs, RGMs, and NTRMs) of the six types of NIST reference materials are certified reference materials (CRMs) under the VIM definition: reference materials, characterized by what NIST 
considers to be metrologically valid approaches for one or more specified quantitative or nominal properties, accompanied by documentation that provides the values of the specified properties, their associated statements of confidence, and statements of metrological traceability [wording adapted from VIM 5.14].

Figure 2 summarizes the relationships among NIST's reference and exploratory materials.

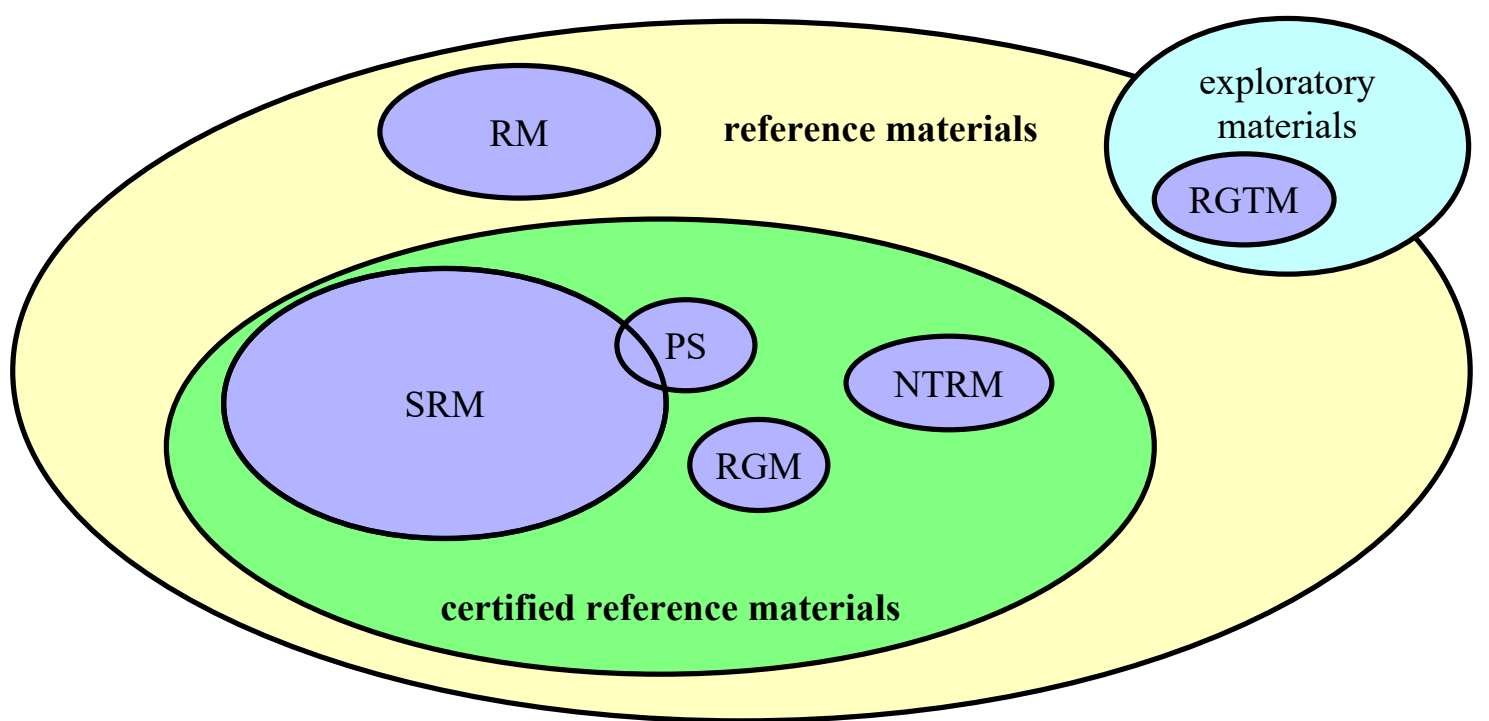

Figure 2. Relationships Among NIST's Reference Materials

The artifact standards identified in the purple ellipses are reference materials (the generic class is indicated by the yellow ellipse) provided by NIST. These materials are all fit for some specified purpose, homogeneous, and stable with respect to one or more specified properties. NIST primary standards (PSs), Standard Reference Materials (SRMs), research gas mixtures (RGMs), and NIST Traceable Reference Materials (NTRMs) are certified reference materials (CRMs, indicated by the green ellipse). These materials deliver assigned values that are suitable for use in method calibration or validation. Note that some SRMs are PSs. NIST's Reference Materials (RMs) deliver fit-for-purpose values that are suitable for between-method harmonization and withinmethod precision and stability assessment. Exploratory materials (light blue ellipse) are fit for the purpose of exploring new measurement challenges; Research-Grade Test Materials (RGTMs) are exploratory materials that have been shown to be fit-for-purpose homogeneous and stable.

\subsubsection{Their Intended Purpose(s)}

The intended purpose of most NIST CRMs is to provide higher-order calibration and validation materials to secondary standard producers and customers intending to produce metrologically traceable in-house calibrants and control materials. ${ }^{15}$ NIST does not have resources to fulfill the global demand for reference materials intended for daily use. While not a change in fundamental policy, this is a change in emphasis. Documents accompanying newly developed reference materials will, more explicitly than in the past, state the purpose that they are intended to serve.

NIST RMs (and non-certified values delivered by CRMs) are intended to support method development and method harmonization for less-well characterized or complex measurands. RGTMs are intended to support measurand discovery and characterization. Interlaboratory studies are used to test a material's fitness for these purposes; stakeholder results inform whether or not to pursue further development. RMs and RGTMs are also suitable for many measurement quality control and assurance applications. 


\subsubsection{Certified Versus Non-Certified Values}

A NIST certified value is a value for which NIST has the highest confidence in that all known or suspected sources of bias and imprecision have been considered and any contributions they may make to measurement uncertainty have been quantified and are expressed in the reported uncertainty. NIST noncertified values are best estimates based on currently available information; however, they do not meet NIST's criteria for certification.

Certified values delivered by a CRM are believed by NIST to be:

- characteristic of the property(ies) specified (measurand),

- characteristic of the material at some defined minimum sample size (homogeneity),

- $\quad$ stable (for some defined period when properly stored and handled),

- accurate (unbiased within a specified level-of-confidence interval),

- metrologically traceable (to a higher-order reference system), and

- documented well enough to provide users with confidence that the certified value is fit for the purpose(s) specified in the documentation supplied to the customer.

These criteria are best thought of as existing along continua from "indisputably inadequate" to "indisputably adequate" where what is "fit-for-purpose adequate" requires decisions informed through good-faith implementation of measurement and quality processes.

NIST certifies a value only when staff believe that all criteria are adequately established. Certification thus explicitly expresses NIST's considered belief that the certified values are suitable for accurately calibrating or validating measurement procedures, which can then be used to obtain values that are metrologically traceable to a higher-order reference system.

NIST provides CRMs that deliver two generic types of certified values: method-independent and method-dependent. A method-independent value comes from a measurement procedure for which the measurand definition is independent of the procedure used to obtain the value. All certification methods that provide metrological traceability to the SI produce method-independent certified values.

The measurand for method-dependent values is operationally defined by the procedure used to obtain the value. As discussed in Section 3.5, there are (at least) two subtypes of these operationally defined measurands: those for which the method is the highest-order reference system (e.g., $\mathrm{pH}$ and Rockwell Hardness Scales) and those that measure a constituent or property that is a proxy for a more difficult to measure constituent or property (e.g., Kjeldahl total nitrogen used to estimate total protein ${ }^{16}$ ).

NIST will not provide any value when NIST staff believe that one or more of the first three of the above criteria (measurand, homogeneity, and stability) are not adequately established. NIST provides noncertified values only when the first three are believed to be adequately established, but one or more of the following three (accuracy, traceability, and documentation) are not.

\subsubsection{Reference, Information, and Indicative Values}

From 1987 until July 2020, NIST non-certified values were called "NIST Reference Values" if a potentially incomplete uncertainty estimate was associated with the value. Values without any associated uncertainty were called "NIST Information Values." Some reference materials from other providers describe non-certified information as "indicative values." NIST does not use this term.

\subsubsection{Documenting Certified Values}

Since NIST asserts that a certified value provides an estimate of the true value of a defined measurand, the documentation provided to the customer clearly differentiates values for method-independent 
measurands from values for operationally defined measurands. When a certified value is associated with measurand that is method-independent, the documentation provided to the customer defines the measurand but needs not provide details of the measurement procedure(s): any valid method that is working properly should return the true value within the stated uncertainty of the material or the uncertainty associated with the measurement process. When a certified value is operationally defined, the documentation defines the measurand and, at minimum, references publicly accessible literature that describes the measurement procedure.

Using any value for its intended purpose requires that customers know how confidence in the value was established. For quantitative values, confidence is expressed as an interval that contains the true value with a stated level of confidence, typically $95 \%$. For nominal values such as measurand identity and for ordinal values such as those used in some measurements of hardness, the documentation briefly describes the criteria used to establish confidence. Only nominal and ordinal values that have been established with "High Confidence" can be certified.

Some users of a NIST CRM might need access to details of the certification in order to use a certified value for their own purposes. The experimental details, data, and data evaluation techniques are made publicly available in a peer-reviewed journal article, a NIST SP 260 or other Technical Series publication, the documentation that accompanies the material (e.g., the Certificate of Analysis), or some combination thereof.

\subsubsection{Documenting Non-Certified Values}

Ironically, non-certified values require that the documentation supplied to the customer provides a much more complete description of the value and its associated confidence than do certified values. To be useful to a customer, the documentation includes:

- The measurement procedure(s) used, in enough detail for an informed customer to understand as appropriate for the NIST-intended use.

- What the value represents; e.g., the mean of samplings from one unit of material, the grand mean of results from multiple units of the material, or the median of an interlaboratory study (ILS).

- What the associated uncertainty or confidence statement represents; e.g., technical precision, repeatability and homogeneity, or reproducibility as estimated from an ILS.

The documentation must not imply that NIST asserts that a non-certified value represents the true value of the measurand. In the documentation that accompanies the material, non-certified values are separated from certified values.

\subsubsection{Other Forms of Information}

When there are values of potential interest to users, but the values cannot be associated with estimates of uncertainty or indications of confidence, these values can be included within NIST CRM documents as part of textual descriptions or in bulleted lists clearly associated with descriptive text. Examples of such data include instrument settings, model parameters, limits of detection, and approximations.

Some NIST CRM documents also provide values in images and plots, such as particle size distributions (e.g., Figure 1, SRM 1649b Urban Dust ${ }^{17}$ ). The documentation should contain enough information about these figures to make clear how the data were obtained. 


\subsubsection{Primary Standards (PSs)}

NIST primary standards (PSs) are suitably characterized materials whose purity values and associated uncertainties are metrologically traceable to internationally recognized higher-order reference systems, typically the SI. The values assigned to PSs are described in NIST internal documentation.

Most materials intended for use as PSs are acquired in small quantities, characterized, and then used to calibrate and to provide metrological traceability to measurement results of other NIST-provided reference materials. These quantity-limited PSs are not available to customers, although some may be shared with collaborating measurement organizations and secondary standards producers in support of national and international measurement comparability.

Several materials such as acetanilide, ${ }^{18}$ cholesterol, ${ }^{19}$ glucose, ${ }^{20}$ and sucrose ${ }^{21}$ are obtained in quantity and characterized for use as PSs that are available for purchase as SRMs (see below). Additionally, the "PSi", (where "i" is an integer) class of PSs are specially characterized NIST CRMs distributed to requesting laboratories, often used as calibrants for characterization of their own calibration standards.

\subsubsection{Standard Reference Materials ${ }^{\circledR}\left(\mathrm{SRMs}^{\circledR}\right)$}

Standard Reference Materials (SRMs) are NIST CRMs that deliver values certified by NIST. All SRMs deliver at least one certified property value that is:

- metrologically traceable to an internationally recognized higher-order reference system, such as the SI, and

- deemed by NIST to be suitable for providing unbiased calibration and/or validation of measurement procedures.

SRMs may also deliver non-certified information. The certified and non-certified values are described in separate sections within the SRM's documentation: a "Certificate" for physical or engineering performance properties and a "Certificate of Analysis" for specific chemical properties.

SRMs are issued as "SRM x" where " $\mathrm{x}$ " is typically a series of digits from 1 to 5999, with an alphabetic or lot number suffix indicating re-issues.

Note: "Standard Reference Material" and "SRM" are trademarks registered to NIST by the United States Patent and Trademark Office.

\subsubsection{Research Gas Mixtures (RGMs)}

Research gas mixtures (RGMs) are specialty gas CRMs produced as single units in collaboration with an industrial or government agency partner. RGMs are certified using the same protocols used for batchcertified NIST gas SRMs. RGMs are identified using the cylinder's identification code. The certified values are described in a "Certificate of Traceability."

\subsubsection{NIST Traceable Reference Materials ${ }^{\mathrm{CM}}$ (NTRMs $^{\mathrm{CM}}$ )}

NIST Traceable Reference Materials (NTRMs) are CRMs that are certified by NIST in collaboration with a secondary standards producer and have a well-defined metrological traceability linkage to existing SRMs or PSs. This linkage is established via criteria and protocols defined by NIST to meet the needs of the community served. Reference materials producers adhering to these requirements can sell the materials using the "NTRM" Certification Mark (CM). The certified values are described in a "Certificate of Traceability." The ongoing specialty gas NTRM program ${ }^{22,23}$ was established in 1992 in partnership with the US Environmental Protection Agency (EPA). A spectrophotometric filter program ${ }^{24}$ was initiated in the late 1990's and ended in 2005. 


\subsubsection{Reference Materials (RMs)}

NIST's reference materials (RMs) deliver property values for established measurands that are fit for the materials' intended uses, including between-method harmonization, within-method precision assessment, and process stability assessment. RMs are issued as "RM 8xxx," where the "xxx" is a unique series of three digits. These materials and values have historically been described in a "Report of Investigation" rather than a Certificate or Certificate of Analysis. For RMs developed after July 2020, these documents are termed "Reference Material Information Sheets."

\subsubsection{Research-Grade Test Materials (RGTMs) and Other Exploratory Materials}

NIST's Research-Grade Test Materials (RGTMs) are a subset of the "exploratory materials" that NIST investigates for use in studying new measurement challenges. RGTMs are issued as "RGTM 1xxxx" where "xxxx" is a unique series of four digits. RGTMs are often first used as test materials in a NISTsponsored ILS. They are prepared to be fit-for-purpose homogeneous and stable but typically have not been quantitatively evaluated for all properties of potential interest. Given enough community collaboration and interest coupled with sufficient funding, results from the study of RGTMs may lead to development of RMs and SRMs. The intention is to rapidly evaluate the fitness of a material for its intended purpose, then as soon as practical release it as an (S)RM with a separate number and label.

Other exploratory materials obtained during the very early stages of an investigation or for use as samples in a single ILS might be useful without meeting the homogeneity and stability requirements for consideration as a reference material. RGTMs differ from single-use exploratory materials primarily in the amount of material purchased and the characterization effort expended prior to their first distribution in an ILS.

At the other end of the reference material life cycle, products that have reached the end of their utility as RMs or SRMs can be repurposed as RGTMs or otherwise used in exploratory research. 


\subsection{Stages in the Reference Material Project Development Process}

Figure 3 displays the typical stages and decision points in RGTM, RM, and SRM development.

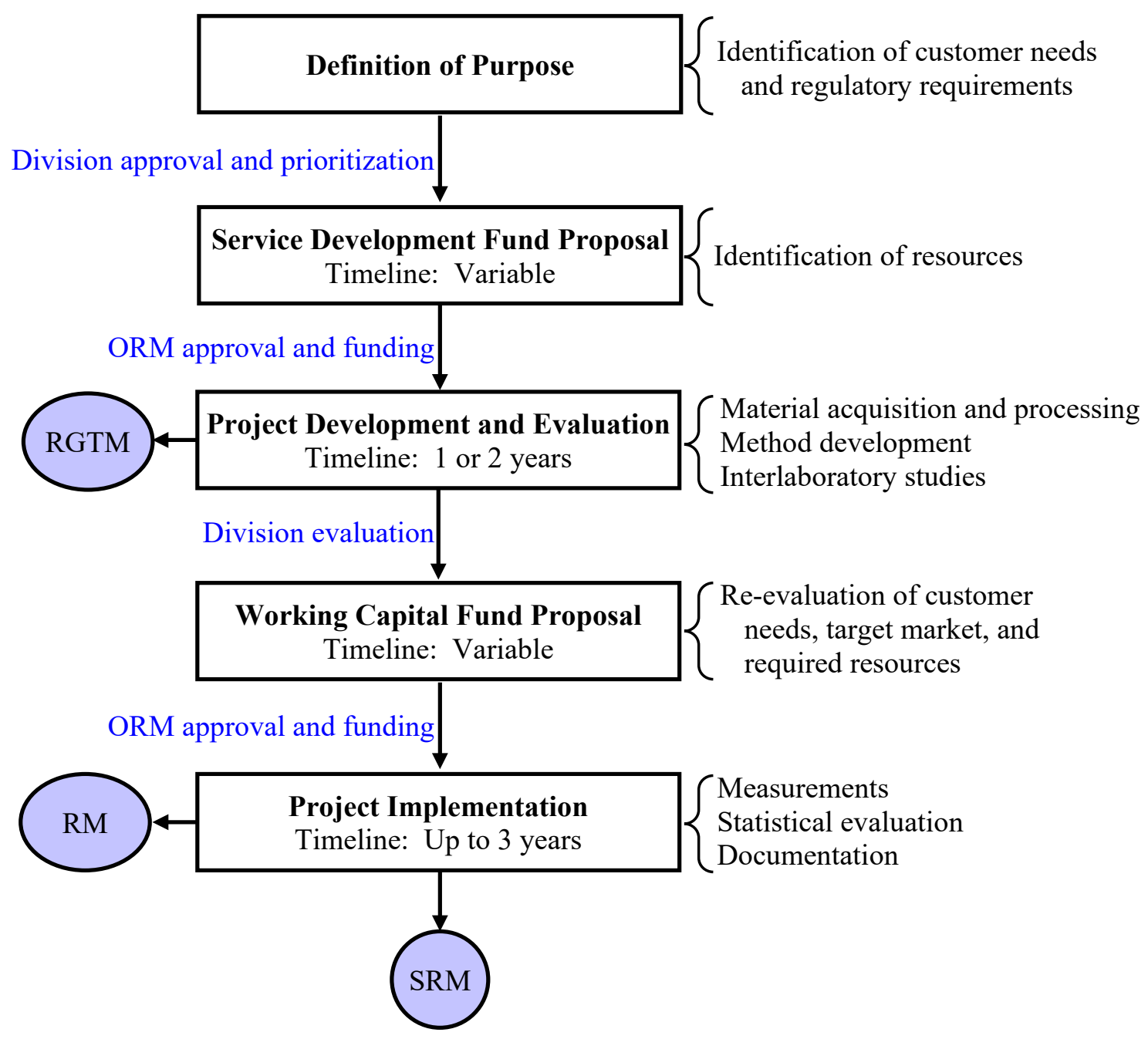

Figure 3. Steps in Reference Material Development

Materials acquired for evaluation and procedure development may become Research-Grade Test Materials (RGTMs). Depending on the purpose of the project, the results from the material evaluations, and availability of resources, some RGTMs may become NIST Reference Materials (RMs) rather than Standard Reference Materials (SRMs) or may be used for other purposes, such as interlaboratory study samples. The NIST Office of Reference Materials (ORM) funds the development of most SRMs through a cost-recovery program. Service Development Fund and Working Capital Fund proposals are typically evaluated annually, but occasionally on demand or by request. Note: "Division" as used here is NIST's designation of the organization of the technical staff who are responsible for providing the required measurement services. 


\section{Definitions and Explanations}

There are numerous sources of metrological terminology. NIST recognizes the third edition of the International Vocabulary of Metrology (VIM), JCGM 200:2012 International vocabulary of metrologyBasic and general concepts and associated terms (VIM3) ${ }^{5}$, ISO Guide 30:2015 Reference materials Selected terms and definitions ${ }^{6}$, and ISO International Standard 17034 General requirements for reference material producers ${ }^{4}$ as authoritative sources, unless they contradict best practices adopted and documented by NIST, in the present document, in the NIST Quality Manual, or in other official NIST publications. These documents are referenced below as [VIM3:section], [ISO Guide 30:2015:section], and [ISO 17034:2016:section].

NIST aims to remain generally consistent with national and international documentary guides, including the International Union of Pure and Applied Chemistry (IUPAC)"s "Gold" 25 and "Green"26 Books and the International Union of Pure and Applied Physics (IUPAP)'s "Red Book"27, but reserves the right to define and use metrological terminology in ways that it deems best, that are consistent with NIST's mission, and that serve U.S. national needs. NIST disseminates such definitions and usage in documents such as this one, and in other NIST publications and documents supporting the NIST Quality Management System.

Note: Metrological terminology, like all living vocabularies, evolves over time. NIST's trademarked "Standard Reference Material" and its acronym "SRM" evolved from the descriptive term "Standard for...," used at the founding of the original organization (Bureau of Standards) to the name "Standard Sample" used until 1967 by NIST's predecessor organization (National Bureau of Standards). As other organizations began to produce similar tools, the international community adopted "certified reference material" (CRM) as the general term. To preserve the NIST brand (and because the term is written into U.S. federal legislation), with the exception of the Research Gas Mixtures (RGMs) and NIST Traceable Reference Materials (NTRMs), NIST-certified CRMs will continue to have the name Standard Reference Material (SRM).

\subsection{Accuracy}

Measurement accuracy is metrologically defined as "closeness of agreement between a measured quantity value and a true quantity value of a measurand" [VIM3:2.13], where "closeness of agreement" is gauged either in absolute terms by a difference or in relative terms by a difference divided by an estimate of the true value. When used to characterize replicate results of a chemical measurement procedure, accuracy is a qualitative term that combines the quantitative concepts of precision and trueness. ${ }^{25}$ Measurement precision characterizes the closeness of agreement of replicate results with each other. Measurement trueness (absence of bias) characterizes the closeness of the mean (or the median, or other suitable indication of location) of the replicates to the true value. Figure 4 displays the interrelationships among the concepts.

When applied to a single measurement value (i.e., one of the red dots in Figure 4), "accuracy" is the same as trueness since there are no replicates to compare. This is related to the common (in non-metrological circles) "accuracy and precision" characterization. However, this usage complicates discussion of "measurement accuracy" until all parties share an understanding of the various concepts.

Although the replication is not explicit, a measurement result expressed as a value and an associated standard uncertainty, $x$ and $u(x)$, can be regarded as summarizing replicate results from a measurement procedure. The $x$ estimates the true value and $u(x)$ expresses and summarizes contributions from all recognized and evaluated sources of uncertainty, including potential bias and lack of repeatability or reproducibility. 


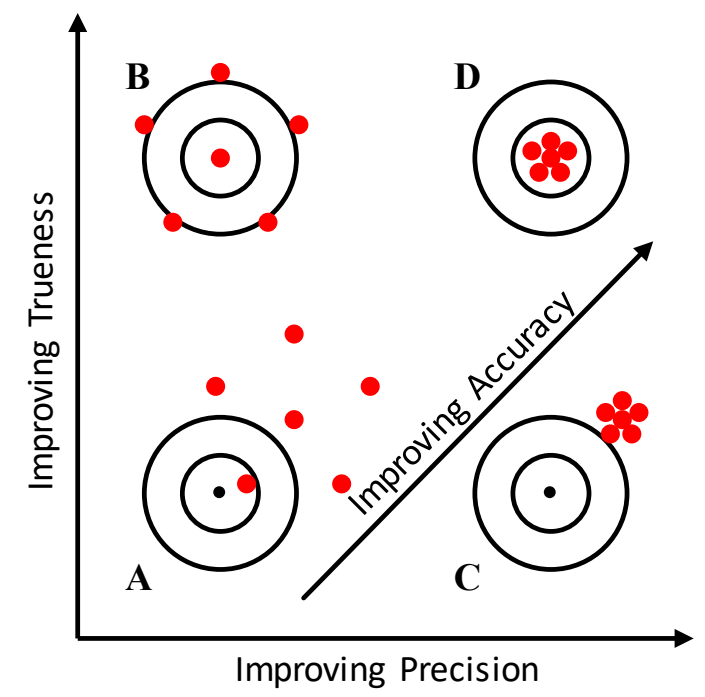

Figure 4. Relationships Between Measurement Accuracy, Precision, and Trueness

The red dots represent individual measurements. The center dot in each target represents the ideal or "true" value for a measurement procedure, where the procedures are: A) imprecise and biased; B) imprecise and true; C) precise and biased; and D) precise and true. Figure adapted from reference [28].

\subsubsection{Precision}

Measurement precision is metrologically defined as "closeness of agreement between ... measured quantity values obtained by replicate measurements on the same or similar objects under specified conditions" [VIM3:2.15]. That is, precision characterizes how well specified types of replicate results agree. Precision improves as the replicate results become closer together (i.e., as the standard deviation decreases), where "closer" can be expressed numerically as the standard deviation of the replicates.

The description of precision depends on the conditions under which the measurements were made. ${ }^{29}$ The most stable condition resulting in the least variability, sometimes called "instrumental" or "technical" precision, characterizes replicate determinations of one fully prepared sample made in the same session. "Repeatability" conditions characterize replicates of independently prepared test portions that are prepared by the same analyst using the same materials and equipment over a relatively short period of time. "Reproducibility" conditions characterize replicate results for samples prepared independently by different analysts in different laboratories using different materials, instruments, and potentially measurement procedures over a relatively long period of time. Conditions more complex than repeatability but less complex than reproducibility are termed "intermediate" with the specific conditions described; e.g., "long-term, within-laboratory, same equipment, different analyst intermediate precision." As shown in Figure 5, imprecision is expected to increase as the conditions become more complex.

While reproducibility precision provides the most realistic estimate of measurement variability, long-term between-analyst intermediate precision is often the most practically realizable estimate that can be achieved without evaluating a material in an ILS. 


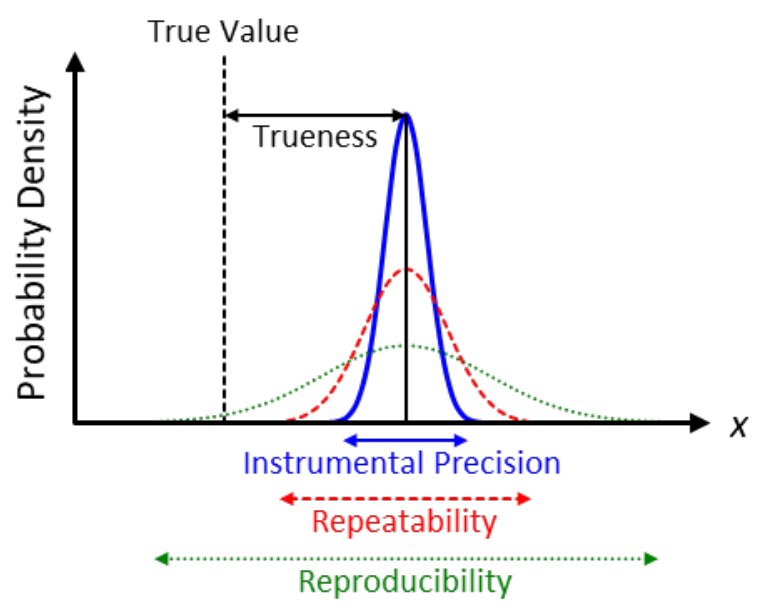

Figure 5. Probability Densities for Precision Conditions at Fixed Trueness

The solid blue curve depicts the narrow distribution expected for instrumental precision, the dashed red curve the distribution expected for measurements made under repeatability conditions, and the dotted green curve the broad distribution expected for measurements made under reproducibility conditions. The vertical dashed line indicates the true value of the measurand; the horizontal arrow connecting the center of the curves and the true value represents the measurement trueness. The arrows below the horizontal axis span the central $95 \%$ of the area underneath each curve.

\subsubsection{Trueness}

Measurement "trueness" is metrologically defined as "closeness of agreement between the average of an infinite number of replicate measured quantity values and a reference quantity value [VIM3:2.14]. Trueness can be colloquially expressed as "lack of bias," characterizing the closeness of agreement between the mean of replicate values and a higher-order reference or "true value." Trueness can be quantified as the difference between the mean and the true value, with trueness improving as the difference becomes smaller. It is often more convenient to speak of bias increasing/decreasing as the difference increases/decreases.

\subsubsection{True Value}

The true value of a quantity or quality is the value that a measurement unaffected by measurement error would produce. While exact knowledge is beyond human reach, the goal of all the modes used to certify SRMs is to provide an estimate of the true value of the measurand to within some specified uncertainty.

\subsubsection{Detection and Quantification Limits}

Measurands that cannot be conclusively detected can be stated as being present at a concentration less than or equal to the detection limit, $x \leq L_{\mathrm{D}} .{ }^{30}$ Measurands that are detected but not reliably quantified can be specified at concentrations greater than the detection limit but less than or equal to the quantification limit, $L_{\mathrm{D}}<x \leq L_{\mathrm{Q}}{ }^{30}$ The information these determinations provide can be combined with other values using appropriate statistical methods 31 .

The value of a limit need not be associated with a further confidence statement. Any uncertainty in determining a limit should be addressed by expanding the stated range. A range may be a certified or a non-certified value depending on how thoroughly it has been characterized.

\subsubsection{Dark Uncertainty}

When measurement results from multiple sources are evaluated, the values sometimes do not agree as well as expected given the estimated uncertainties (i.e., the error bars don't overlap). Such excess 
variability is often called "dark uncertainty" ${ }^{32}$. The presence of dark uncertainty indicates that there are unrecognized or under-estimated sources of bias and/or imprecision among the measurement processes. If the sources cannot be identified and corrected, estimating reliable consensus values requires use of appropriate statistical approaches ${ }^{33}$.

\subsection{Collaborators}

ISO Guide 30:2015 equates "collaborator" with "subcontractor," which is defined as "body (person, organization, or company, public or private) that undertakes aspects of the processing, handling, homogeneity, and stability assessment, characterization, storage, or distribution of the [reference material] on behalf of the [reference material] producer, on a contractual basis, either paid or non-paid" [adapted from ISO Guide 30:2015:4.6]. However, this document restricts the term to apply only to organizations or individuals who help characterize NIST materials. This document recognizes three types of collaboration based on the level of engagement with NIST.

\subsubsection{International Peer Organizations}

Measurements made by many national metrology institutes (NMIs) and designated institutes (DIs) may have formal equivalency with those made by NIST through the CIPM Mutual Recognition Arrangement (MRA). ${ }^{34}$ While DIs typically have more limited responsibilities than do NMIs, NMIs and DIs are both at the top of their national calibration hierarchies for defined types of measurements. These organizations typically interact with NIST through comparison studies authorized by the CIPM and conducted by the Consultative Committee responsible for the relevant metrology area. The Consultative Committee for Mass and Related Quantities (CCM) is responsible for supporting comparisons involving hardness measurements. The Consultative Committee for Amount of Substance: Metrology in Chemistry and Biology (CCQM) is responsible for supporting comparisons involving chemical and biological measurands.

\subsubsection{Expert Collaborators}

In this document, the unofficial term "expert collaborator" is used for organizations other than NMIs and DIs that engage with NIST through direct comparison of measurement capabilities through bilateral or small multi-center studies designed to address specific issues. The organizations include:

- United States governmental agencies such as the Centers for Disease Control and Prevention (CDC), Food and Drug Administration (FDA), National Center for Atmospheric Research (NCAR), U.S. Geological Survey (USGS), and U.S. Department of Agriculture (USDA).

- International agencies such as the International Bureau of Weights and Measures (BIPM), International Atomic Energy Agency (IAEA), World Anti-Doping Agency (WADA), and World Meteorological Organization (WMO).

- Standards development organizations that validate the standard test procedures, practices, and guides they produce. Examples include the standard test development committees of AOAC International, ASTM International, and the Clinical and Laboratory Standards Institute (CLSI).

- Individuals and organizations in academia, government, and the private sector that have the needed capabilities, are willing to contribute their efforts, and are willing to perform experiments in a way that satisfies requirements of the NIST Quality Management System.

\subsubsection{Participants in Interlaboratory Studies}

Consensus results from ILSs are a valuable and increasingly important source of data used to help NIST characterize materials. While the participants in such studies are collaborators, they need not be measurement experts if there are enough participants in the study to provide reliable consensus estimates. What is considered "enough" depends on how different the results are, the data analysis methods used, and whether the ILS included control materials. 


\subsection{Fitness for Purpose}

"Fitness for purpose" is a vital metrological concept, but it is not formally defined. Rather, it addresses the straightforward question "Does this instrument or procedure or material adequately meet the requirements of the proposed use?"

Fitness is necessarily a relative concept, because what is fit for one purpose may be unfit for even closely related purposes. For example, an SRM designed to support the measurement of chromium in a sediment using procedures that consume $500 \mathrm{mg}$ samples may not be sufficiently homogeneous to support microanalysis methods that are limited to no more than $5 \mathrm{mg}$. What was fit for purpose yesterday may not be fit for the identical purpose tomorrow if regulatory action limits are changed.

In general, the intended purpose of NIST's SRMs is to provide higher-order calibration and validation materials to secondary standard producers and customers intending to produce metrologically traceable in-house calibrants and control materials. NIST's SRMs are fit for their intended purpose if they deliver to the intended customers "true enough" values for useful levels of the desired measurands in a timely manner and at reasonable cost. SRMs that are not adequately fit for customer purpose(s) are not useful even when all design criteria are satisfied. It is therefore imperative that new and renewal SRMs be designed relative to the needs and capabilities of properly targeted customers. It is a waste of effort and resources to produce a material that is "better" - and consequently more expensive to certify and to sell than what the customer requires.

\subsection{Interlaboratory Study (ILS)}

For use with the "Certification Modes" described in Section 3, an ILS is any study involving multiple organizations that provides enough technically valid measurement results to enable generation of a fit-forpurpose consensus value with an associated uncertainty. Such a consensus value and uncertainty may then be used to help certify values without NIST having specific knowledge of the participants' measurement processes. If the study has been appropriately designed to enable confident identification of technically valid results, NIST need not be a coordinator of nor a participant in an ILS to use the reported information.

When the ILS yields fewer than five technically valid results, NIST must have enough information about the methods and calibrants used to achieve those results so that a summary report can be prepared that satisfies the NIST Quality Management System. The collaborators do not need to provide formal written reports as long as NIST receives the needed information.

\subsection{Higher-Order}

Another important concept that is not formally defined, "higher-order" is used often in metrological contexts. The term usually indicates that whatever the compound adjective modifies is "among the best" (or "the best") currently available within a given hierarchy. For example:

- Higher-order metrological traceability Traceability to the top link in an unbroken chain of comparisons, typically to the SI.

- Higher-order materials Materials that provide higher-order metrological traceability when used as calibrants. PSs are higher-order CRMs while CRMs are higher-order reference materials.

- Higher-order reference measurement procedures (RMPs)

Reference measurement procedures (RMPs) that provide accurate results traceable to a higherorder reference system through a minimal number of linkages. Coulometric titrimetry, gravimetry, isotope-dilution mass-spectrometry (ID-MS), and quantitative nuclear magnetic resonance (qNMR) are examples of higher-order RMPs that do not require calibration with a 
calibrant of the same kind as the sample. However, many analytical procedures can be of higherorder simply by calibrating with a higher-order calibrant of the same kind as the sample.

\section{- Higher-order measurement results}

Results produced using higher-order procedures, validated by comparison to measurements made on higher-order reference materials. Certified values are higher-order results.

- Higher-order reference systems

Traditionally, NIST certified values have exclusively been metrologically traceable to the SI. However, there are internationally recognized material- and procedure-based reference systems for measurands where traceability to the SI is impractical or irrelevant. Examples include the World Health Organization's materials that define international units of biologic activity ${ }^{35}$, isotopic $\delta$-scales for expression of stable-isotope-ratio measurements ${ }^{36}$, and the International Association for Cereal Science and Technology's ICC Standard No. 185 procedure for measurement of dietary fiber. ${ }^{37}$

\subsection{Homogeneity and Stability}

To be useful, any PS, SRM, RGM, NTRM, RM, or RGTM must be fit-for-purpose homogeneous and stable for the period of validity. However, homogeneity (uniformity of composition within a material) and stability (uniformity of composition over time) are relative, not absolute, concepts.

\subsubsection{Homogeneity}

Homogeneity is metrologically defined as "uniformity of a specified property value throughout a defined portion of a reference material" [ISO Guide 30:2015:2.12]. ISO 17034:2016:7.10 describes requirements for assessing the homogeneity of reference material constituents. An analyte dispersed in a granular material may appear to be uniformly dispersed if suitably large samples are analyzed but may be quite heterogeneous (as a result of "nuggets," individual granules enriched in one or more analytes) ${ }^{38}$ when smaller sample sizes are used. At any given sample size, the uniform dispersal of one analyte does not necessarily indicate that the other analytes in the material are homogeneously distributed, especially those that do not have similar physicochemical properties. Materials that are suitably homogeneous for relatively imprecise measurement procedures may not be so for more precise procedures.

Homogeneity is not just an issue for solids; gases and liquids can stratify within their containers. However, assessing the homogeneity of a solid reference material generally requires more complex procedures that depend on the form of the material: e.g., disk, rod, chip, granule, or powder.

For all reference material forms, it is critical that the intended purpose for the material is well-defined and the material is prepared for that purpose. The development process must define the storage conditions, handling practices, mixing and sampling practices, and minimum sample size that will satisfy the purpose of the reference material.

\subsubsection{Stability}

Stability is metrologically defined as "characteristic of a reference material, when stored under specified conditions, to maintain a specified property value within specified limits for a specified period of time" [ISO Guide 30:2015:2.15]. There are two major aspects to stability for NIST-issued reference materials: 1) while stored at NIST and under our control and 2) during shipment.

Note: The documentation supplied with NIST CRMs states required storage conditions. Storage under these condition upon receipt is the responsibility of the customer. 


\subsubsection{Transportation Stability}

Transportation stability, often referred to as "short-term stability," is "stability of a reference material property for the time period and conditions encountered in transportation to the user of the reference material" [ISO Guide 30:2015:2.16]. ISO Standard 17034 requires producers to "assess, by experimentation if necessary, the stability of all relevant properties of [a reference material] under proposed conditions of transport, and choose transport conditions to maintain stability during transport" [ISO 17034:2016:7.11].

NIST's packaging and shipping processes are intended to minimize the opportunity for instability while complying with any hazardous material packaging and shipping regulations. However, the stability of materials thought to be prone to degradation should be evaluated under realistic conditions (e.g., vibration, temperature, freeze-thaw cycles). If degradation is found likely, special handling instructions may be required and an additional component included in the uncertainty budget for any affected property value to account for potential degradation during shipment.

\subsubsection{Storage Stability}

Often referred to as "long-term stability," storage stability is "stability of a reference material property over an extended period of time" [ISO Guide 30:2015:2.17]. ISO Standard 17034 requires producers to "assess, by experimentation if necessary, the stability of all relevant properties of [a reference material] under proposed storage conditions and choose pre-treatment, packaging and storage conditions in accordance with the results of the assessment" [ISO 17034:2016:7.11]. An important step in evaluating the uncertainty of an assigned property value is to consider including a component of uncertainty to account for potential degradation during long-term storage.

For many inorganic analytes, the amount of substance content in matrices such as metals and ores is unlikely to change much over even long time periods regardless of handling and storage conditions. For some inorganic analytes in solution and many organic analytes in any matrix, the contents can change rapidly, even when materials are carefully handled and stored. Analytes in mixtures can react with each other, their matrices, or their packaging, causing the amount of substance to decrease or increase over time.

Compositional changes that are fit for a single, quickly completed ILS might not be fit for materials that will be stored for years before sale.

It is impractical to experimentally measure the compositional stability of every constituent of interest in even a single complex material on a regular basis. NIST therefore monitors its products during their period of validity and, if substantive technical changes occur that affect the values, takes corrective action and notifies users and purchasers. The following are examples of monitoring techniques that help to ensure that NIST is aware of changes during the lifetime of the product:

- Scheduled evaluation against primary standards of selected constituents, typically in relatively new materials.

- Evaluation through use of materials as controls during the development of a reissue or new reference material of similar matrix. For example, Figure 6 displays results of NIST measurements made during the past 19 years for selected elements in SRM 1566b Oyster Tissue. While the analytical quality of individual values may differ, collectively they can provide reliable evidence that at least most of these analytes are stable in complex organic matrices over long periods of time.

- Evaluation through use of materials as ILS controls and/or unknown samples. Figure 7 displays consensus values for total $\beta$-carotene in the component sera of four reissues of SRM 968 Fat-Soluble Vitamins in Human Serum. Distributing the same materials in multiple studies of 
similar nature directly assesses stability not only in the materials themselves and the distribution processes but also in future materials of similar matrix.

- Scientific judgment about the expected behavior of constituents in similar materials informed by experience with similar measurands. Since short-term studies established that trans- $\beta$-carotene is a reasonable proxy for other fat-soluble vitamin-related analytes in serum, ${ }^{39}$ the long-term stability evidence provided in Figure 7 is believed to extend to the other carotenoids in these same sera.

- Continuous interaction with customers and rapid responses to inquiries. As part of NIST's Quality Management System, ${ }^{7}$ customer inquiries and comments are addressed promptly and thoroughly. Complaints trigger a review of available data and, if the potential deficiency is found credible, action is taken to identify its cause, develop a remedy, and determine if any other product is affected.

When the validity of a certified value becomes suspect, the material is "put on restriction" and sales are ceased until the issue is resolved by direct experimental measurements.

Note: While NIST's customers sometimes incorrectly attribute issues with their measurement procedures to issues with the materials, they also sometimes fail to recognize that a discordance may indicate a real problem with the material or its certificate; ${ }^{40}$ therefore inquiries are always welcomed.

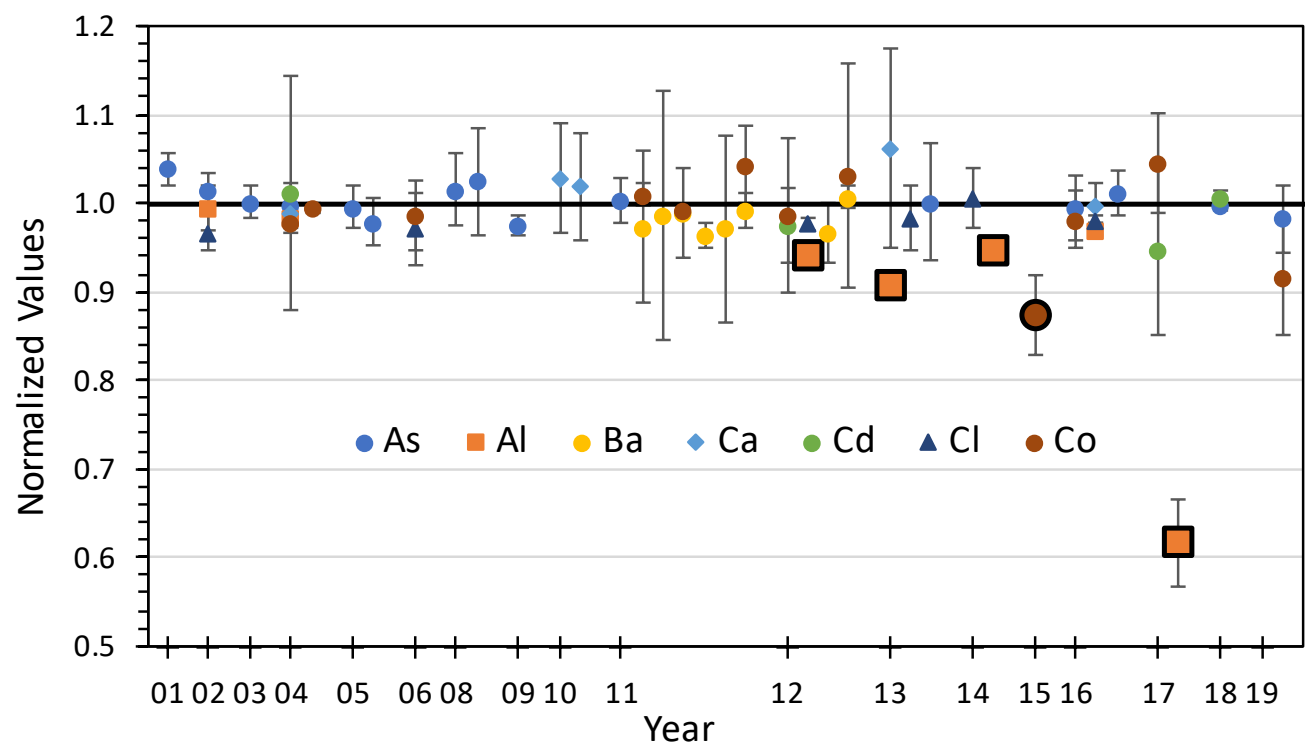

Figure 6. Stability of Selected Elements in SRM 1566b Oyster Tissue

Each symbol represents a NIST measurement, $x \pm u(x)$, of SRM 1566b used as a control to help validate measurements of another material. All data are normalized to the analyte's certified value. The large symbols with the black border (four $\mathrm{Al}$ and one $\mathrm{Co}$ ) denote results that do not adequately agree with the certified value. Based on the erratic pattern of the excursions over time and the absence of correlated excursions between elements with similar properties, the excursions likely represent measurement rather than material stability issues. Figure courtesy of Savelas A. Rabb, NIST. 


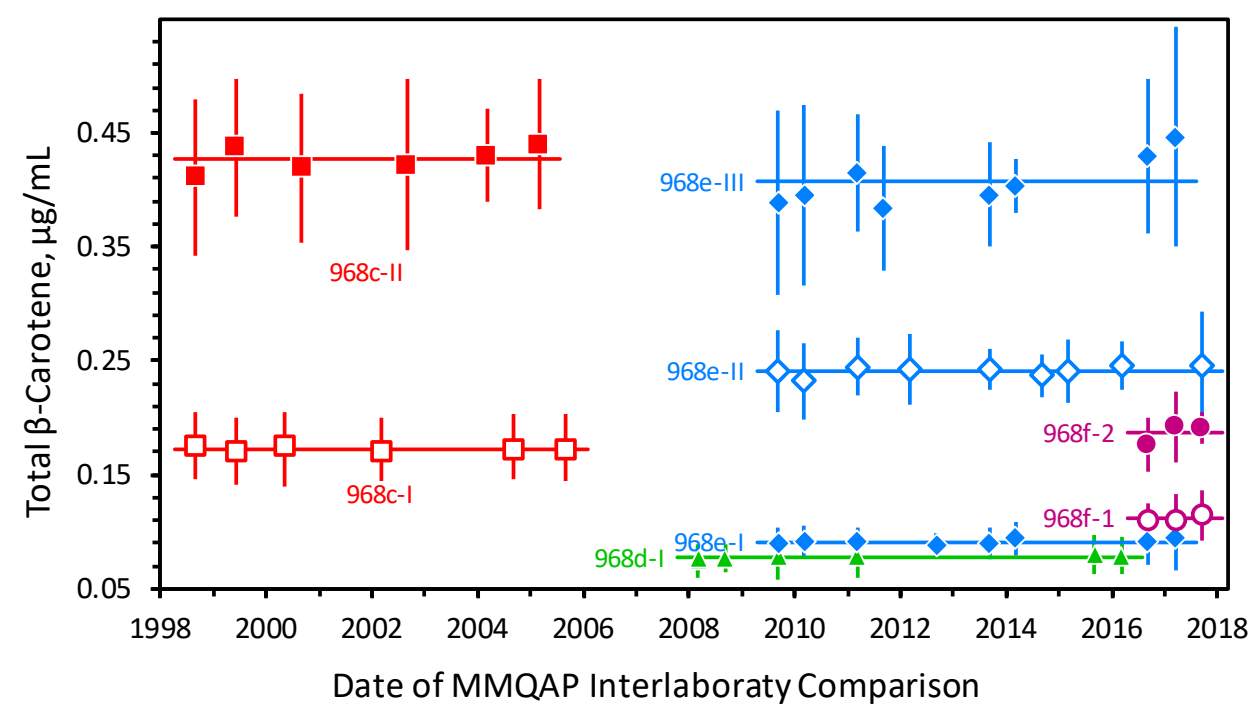

Figure 7. Stability of Total $\beta$-Carotene in Frozen Human Serum

Each symbol represents the consensus ILS result, $x \pm u(x)$, for total $\beta$-carotene in one serum of the SRM 968 Fat-Soluble Vitamins in Frozen Human Serum series. The horizontal lines represent the mean of the individual results. These materials were distributed as unknowns in the NIST-coordinated Micronutrients Measurement Quality Assurance Program (MMQAP). ${ }^{117}$

\subsubsection{Period of Validity}

A period of validity is the time during which certified values are expected to remain correct within the stated uncertainty. NIST makes reasonable efforts to verify the stability of the material and its constituents and properties. For most materials, the period of validity will be a specific number of years, estimated from stability testing and experience with similar measurands in similar matrices. For some materials, such as metals and most alloys, the period of validity may be indefinite based on scientific knowledge and experience with the materials that assures the true values of the measurands are expected to remain within their stated uncertainty intervals for the lifetime of the purchaser. In all cases, due diligence is required to justify the designation and to provide instructions for storage, handling, and use that can be expected to help maintain stability. Periods of validity may be extended based on verification of material stability.

\subsubsection{Expiration Date}

Unless the period of validity is indefinite, the documentation provided with each reference material states an expiration date after which the assigned values are not supported. The expiration date is based on the established period of validity.

Most SRMs and RMs are put on restriction (i.e., sales are ceased) three months prior to the expiration date; gases are restricted one year prior to their expiration date. (These restrictions are for purposes of internal stock control; they do not affect the period of validity.) If the amount of stock, customer needs, and availability of staff and instrumentation justify the effort, resources are devoted to determining whether the period of validity can be extended. If there is adequate evidence of stability, the expiration date stated in the documentation is updated.

Note: Customers are not notified of an extended expiration date. It is up to the customer to check that their documentation is current. This can be accomplished online at https://www.nist.gov/srm or by contacting the SRM Program: telephone (301) 975-2200; fax (301) 948-3730; e-mail srminfo@nist.gov. 


\subsubsection{Revision History}

The documentation (i.e., Certificate of Analysis, Reference Material Information Sheet, etc.) for NIST's reference materials may undergo revision during the period of validity. Changes include editorial revision, extension of the period of validity, upgrading non-certified values to certified status, downgrading certified values to non-certified status, and removing values. A revision history is provided, typically on the document's last page.

\subsection{Material Handling and Storage}

Tracking and ensuring the integrity of materials and data throughout a reference material development process is fundamental to successfully delivering values that are fit for their intended purposes. ISO 17034:2016:7.4 specifies requirements for all materials. ISO 20387:2018 Biotechnology Biobanking - General requirements for biobanking ${ }^{41}$ specifies requirements for biological materials.

\subsection{Measurand}

A measurand is the quantitative or qualitative property that is the object of measurement. VIM3:2.3 defines a quantitative measurand as the "quantity intended to be measured," and supplements this definition with these Notes, which also apply to qualitative measurands, with obvious modifications:

- specifying a measurand requires knowledge of the kind of quantity, description of the substance carrying the quantity, relevant components of the substance, and the chemical entities involved.

- while sometimes used as a synonym, "analyte" refers to a chemical entity not a quantity.

The distinction between analyte and measurand is discussed at length in reference ${ }^{42}$, along with the logic behind the current definition's evolution from the "quantity subject to measurement" definition given in the second edition of the VIM (VIM2). ${ }^{43}$

Note: The measurand is not necessarily what is measured. For instance, measurement of the analyte total nitrogen using the Kjeldahl method ${ }^{16}$ is traditionally used to value assign the measurand total protein content of many foods.

It is critical that the measurand be (fit-for-purpose) completely specified for a value to be considered certifiable. Most chemical measurands are specified as the type of quantity (e.g., mass fraction expressed as $\mathrm{mg} / \mathrm{g}$, mass concentration expressed as $\mu \mathrm{g} / \mathrm{mL}, \mathrm{pH}$ ) of the target analyte (e.g., iron, total retinol, conventional activity of the hydronium ion) in a matrix (e.g., nitric acid at an amount of substance concentration of about $1.6 \mathrm{~mol} / \mathrm{L}$, normal human serum, aqueous solution prepared from solids according to a specific protocol). Describing a value as the "mass fraction of hexavalent chromium in aqueous industrial effluent evaluated using EPA Method 7196A" is much less open to misinterpretation than is "concentration of chromium in water."

\subsubsection{Nominal and Ordinal Properties}

Nominal and ordinal properties are qualitative properties, often called "categorical properties" ${ }^{44}$ when taken together because their values are names of categories, or classes, whose elements have particular values of defining properties in common (the defining properties may be qualitative or quantitative). Being a rare-earth or an alkali metal is a nominal property of the chemical elements. Determining whether a compound includes a rare-earth element may involve both quantitative and qualitative measurements. Being acidic is a property of an aqueous solution, which may be determined using a $\mathrm{pH}$ indicator.

The values of ordinal properties can be ranked (ordered from smallest to largest) yet they are not quantitative - of them it can be said only whether one is more or less than another, but not by how much. Blue litmus ${ }^{45}$ paper will turn red when dipped in an acidic solution, and will remain blue in an alkaline 
solution, thus indicating only that the $\mathrm{pH}$ of the former is lower than the $\mathrm{pH}$ of the latter. Even when the values of ordinal properties are expressed numerically, as they are in the Mohs hardness scale ${ }^{46}$ for minerals, the numbers serve only to indicate rank order, being otherwise unsuitable for including in arithmetic operations.

NIST's understanding of "measurement" includes value-assignment to qualitative or quantitative properties. ${ }^{47}$ Both VIM3 and ISO Guide 30:2015 recognize that nominal properties are measurands that can be value-assigned using the same concepts as traditional, quantitative measurands, and whose associated uncertainties may be expressed quantitatively:

- “'uncertainty' covers both 'measurement uncertainty' and 'uncertainty associated with the value of a nominal property,' such as for identity and sequence. 'Traceability' covers both 'metrological traceability of a quantity value' and 'traceability of a nominal property value' [VIM3:5.14 Note 2].

- "The concept of value includes a nominal property or a qualitative attribute such as identity or sequence. Uncertainties for such attributes may be expressed as probabilities or levels of confidence" [ISO Guide 30:2015:2.2 Note 1].

Since at least 1964, NIST has provided CRMs that deliver nominal properties. Standard Sample 2106 ISCC-NBS Centroid Color Charts ${ }^{48}$, a collection of 251 paint-on-paper samples, illustrated a set of standard colors. SRM 1810 Linerboard Standard for Tape Adhesion Testing ${ }^{49}$ delivers a surface that adhesive tape either does or does not stick to under defined conditions. SRM 1196a Standard Cigarette for Ignition Resistance Testing ${ }^{50}$ delivers cigarettes that either do or do not extinguish before burning their whole length. These and related properties are typically metrologically traceable to specified scales or test methods.

\subsubsection{Identity}

The identity of a chemical element or compound, rock or mineral species, or living organism is a nominal property. NIST recognizes that identity is a nominal property that can be delivered by reference materials, and that the associated uncertainty can be expressed either quantitatively or in an ordinal scale. ${ }^{51,52}$ For example, SRM $3246^{53}$ assigns the species value Ginkgo biloba to the material, based on identifying sequences of nucleotides in its DNA, and expresses the associated uncertainty qualitatively, in an ordinal scale that represents the strength of the belief in the assigned value.

Chemical identity may be regarded as being metrologically traceable to the natural chemical structure of a compound. The degree of structural specificity of the measurand depends on the purpose, e.g., whether or not isomeric forms present need to be distinguished. Biological identity can be regarded as being traceable to characteristic DNA or amino acid sequences of the holotype (type specimen) of the organism, available in internationally recognized databases. The requirements for adequately documenting traceability to these standards are critically dependent on the needs and agreement of the relevant measurement communities. 


\subsubsection{Surrogate Measurands}

Occasionally SRMs deliver certified values for measurands that are surrogates for measurands that are quite different from, but functionally related to, those known to be of greatest interest to the user. For example: SRM $17 \mathrm{f}$ Sucrose $^{21}$ is intended for use as a saccharimetry standard in calibrating polarimetric systems, while the certified value is chemical purity; SRM 141e Acetanilide ${ }^{16}$ is intended for use in validating microchemical procedures for the determination of carbon, hydrogen, and nitrogen in organic matter, while the certified values are chemical identity and purity. For such materials, the measurands of primary interest should be well-enough characterized to confirm that the material is fit for purpose. Also, the materials need to be characterized for impurities and other interferences that would compromise their fitness. In both examples, the certified values were established through measurements made by NIST, non-certified values characteristic of their intended purpose calculated using established relationships, and fitness-for-purpose confirmed by measurements provided by collaborators.

Similarly, certified values for the measurands of primary interest are sometimes based on measurements of suitable surrogates. For example, the metals in SRM 1880b Portland Cement ${ }^{54}$ are certified as oxides but were measured as elements; SRM 3154 Sulfur Solution (Lot 892205) ${ }^{55}$ is certified for sulfur content but the measurements were coulometry and gravimetric precipitation of sulfate ion. For such materials, it is necessary that the stochiometric relationships between what is measured and what is certified be well established. In both examples, the source and preparation of the materials provides the necessary fit-forpurpose evidence.

\subsection{Measurement Method, Procedure, and Process}

While it is common practice to describe specific and detailed measurement protocols as "test methods," a measurement method is officially a "generic description of a logical organization of operations used in a measurement" [VIM3:2.5]. A measurement procedure is a "detailed description of a measurement according to one or more measurement principles and to a given measurement method, based on a measurement model and including any calculation to obtain a measurement result" [VIM3:2.6].

This document uses the term process to encompass how analytical information from multiple measurement procedures and studies is combined to produce measurement results. Such processes typically involve several pre-analytical steps (e.g., sampling, storage, transport) required to obtain a characteristic subsample of a test material; pre-measurement steps (e.g., extraction, chemical modifications, dilution, preparation of subsamples and validation materials) to convert the subsamples to forms suitable for analysis with one or more measurement procedures; calibration; measurement of the sample and validation materials, including homogeneity and stability studies; interpretation of the data; and calculation of the measurement result. Figure 8 outlines the generic steps, and the typical flow of information between the steps, for processes that are intended to produce certified values. 


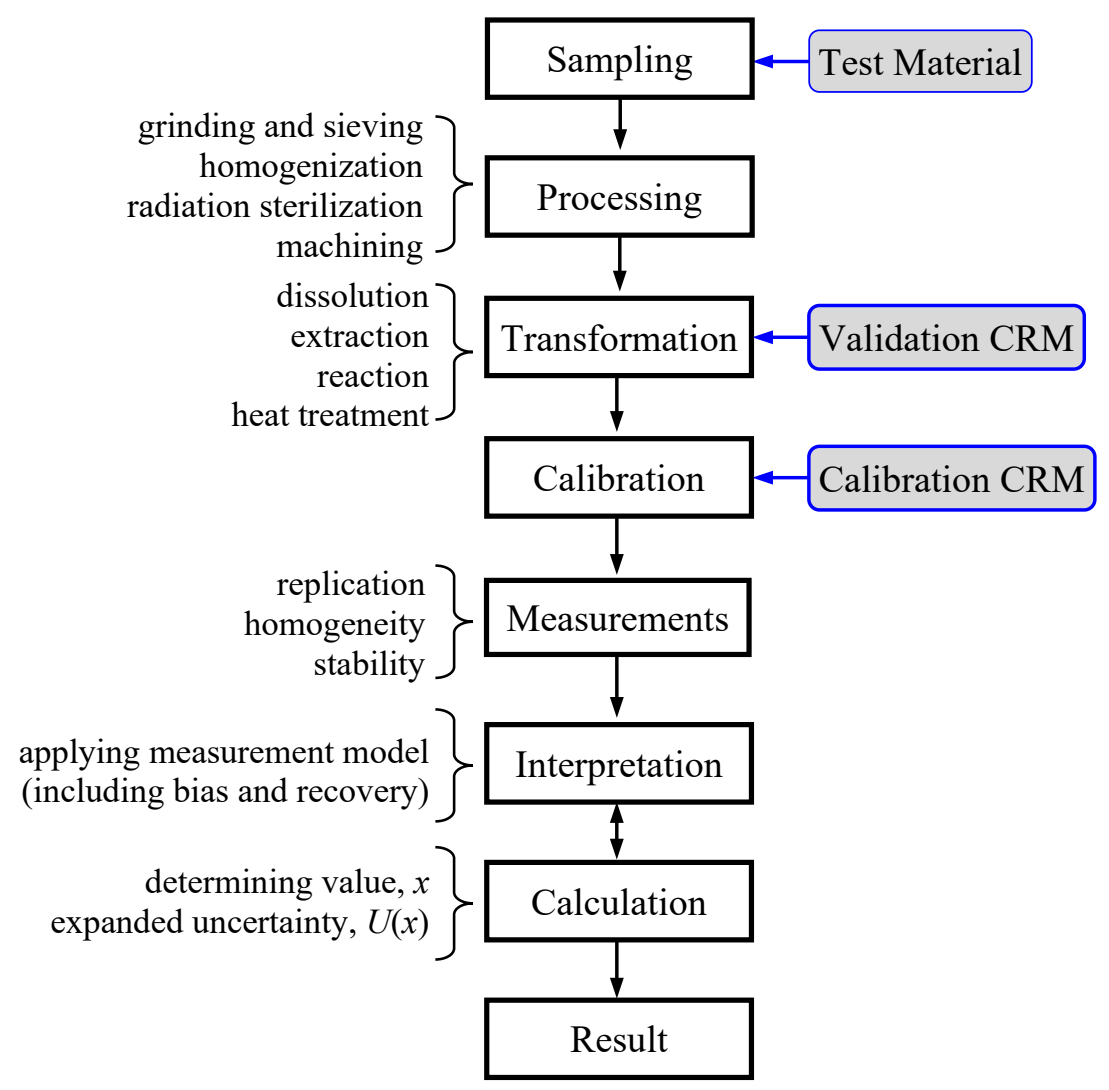

Figure 8. Steps in a Generic Measurement Process for Certifying Values

The grey shapes to the right indicate the step at which the test material, validation CRMs, and calibration CRMs typically enter the process. The entries to the left are examples of activities associated with the respective stages of the process. The arrows that connect the steps represent transfer and often storage of materials and data. In practice, interpretation and calculation often proceed iteratively. 


\subsubsection{Method Calibration}

Calibration is formally defined as an "operation that, under specified conditions, in a first step, establishes a relation between the quantity values with measurement uncertainties provided by measurement standards and corresponding indications with associated measurement uncertainties and, in a second step, uses this information to establish a relation for obtaining a measurement result from an indication" [VIM3:2.39], where indication is defined as a "quantity value provided by a measuring instrument or a measuring system" [VIM3:4.1]. That is, calibration characterizes how a measuring instrument responds (the readings and associated uncertainties that it produces) when presented with particular values of the measurand that are surrounded by stated uncertainties. The mathematical inverse of the calibration function is often called the "analysis function," which translates instrumental indications (responses) into values of the measurand.

Figure 9 displays the elements of calibration for a linear relationship based on an idealized chromatographic analysis for 24R,25-dihydroxyvitamin $\mathrm{D}_{3}$ calibrated with SRM 2971

24R,25-Dihydroxyvitamin $\mathrm{D}_{3}$ Calibration Solution. ${ }^{12}$ This SRM delivers the analyte at a certified mass fraction of $(1.054 \pm 0.019) \mu \mathrm{g} / \mathrm{g}$, about a factor of 200 times greater than the expected range in normal human sera. The working calibration solutions are prepared by gravimetric dilution into the solvent used to prepare routine samples. In addition to the uncertainty in the certified value, these preparatory steps contribute to the uncertainty in the mass fraction of analyte in the working standards.

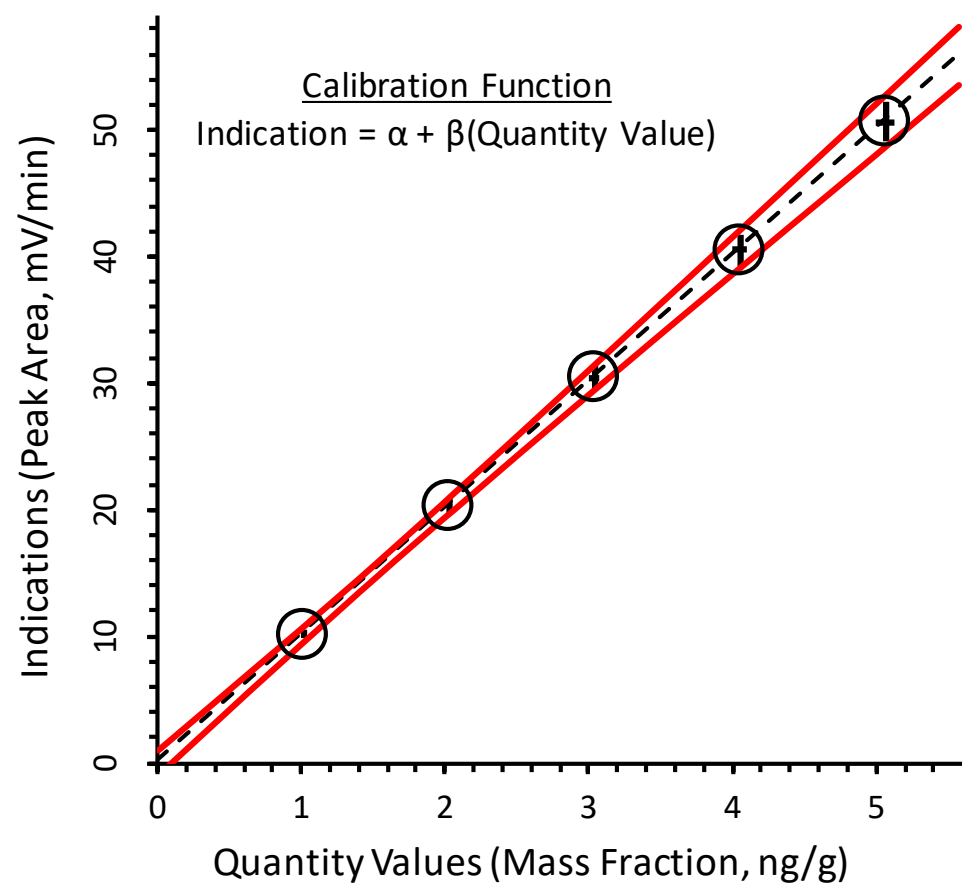

Figure 9. Quantity Values, Indications, and Calibration Function

The crosses with the open circles represent $95 \%$ expanded uncertainties on the measured indications (here, chromatographic peak areas measured in $\mathrm{mV} / \mathrm{min}$ ) and calibration points (here, solutions prepared by gravimetric dilution of an SRM with analyte mass fractions expressed in $\mu \mathrm{g} / \mathrm{g}$ ). Calibrant quantities are plotted along the horizontal $(\mathrm{X})$ axis; indications (instrument responses) are plotted along the vertical (Y) axis. The dashed black line represents the linear calibration function: $Y=\alpha+\beta X$. The solid red curves represent the $95 \%$ uncertainty interval on the function. In this example, the values of the $\alpha$ and $\beta$ parameters are $(0.04 \pm 0.50) \mathrm{mV} / \mathrm{min}$ and $(10.00 \pm 0.30)(\mathrm{mV} / \mathrm{min}) /(\mathrm{ng} / \mathrm{g})$, respectively; the correlation between the $\alpha$ and $\beta$ parameters, $r(\alpha, \beta)$, is -0.85 . 
Note: Classical regression (the technique widely available in spreadsheets) assumes the values of the independent variable(s), $x$, are known exactly and those of the dependent variable, $y \pm u(y)$, are probability distributions. Therefore, classical regression represents indications as a function of known quantities: $Y=\alpha+\beta X$. The inverse of the calibration function, here $X=(Y-\alpha) / \beta$, is then used to predict the quantity of an unknown from its measured indication. Various implementations of generalized distance regression (GDR) (also called generalized least squares (GLS), total least squares (TLS), errorsin-variables (EiV), etc.), where both the dependent and independent variable are defined as probability distributions rather than fixed values, are available but require specialized software. ${ }^{56,57,58,59}$ GDR treats instrumental indications and the quantity values of the measurand in the calibrants symmetrically, hence affords the opportunity to build the analysis function directly, instead of as the mathematical inverse of the calibration function. NIST employs this approach routinely for value assignment and uncertainty quantification for gas mixture CRMs. ${ }^{56}$

\subsubsection{Method/Procedure/Process Validation (and Verification)}

The VIM defines validation as "verification, where the specified requirements are adequate for an intended use" [VIM3:2.45] where verification is defined as "provision of objective evidence that a given item fulfills specified requirements" [VIM3:2.44]. ASTM E2857 Standard Guide for Validating Analytical Methods provides a more pertinent definition: "confirmation, by the provision of objective evidence and examination, that a method meets performance requirements and is suitable for its intended use." ${ }^{60}$ As used in this document, validation and verification are regarded as synonyms. Both terms refer to the collection, documentation, and presentation of evidence that the performance of a $\mathrm{method} /$ procedure/process is fit for purpose.

Figure 10 displays one approach to validation, treating a validation CRM as an unknown and comparing the measurement result with the certified value. A sample of SRM 2973 Vitamin D Metabolites in Frozen Human Serum (High Level) ${ }^{96}$ is analyzed as an unknown. The certified 24R,25-dihydroxyvitamin $\mathrm{D}_{3}$ mass fraction delivered by this material is $(3.06 \pm 0.11) \mathrm{ng} / \mathrm{g}$. The peak area is evaluated as $(30.6 \pm 1.1) \mathrm{mV} / \mathrm{min}$. Using the calibration function summarized in Figure 9, the mass fraction is estimated to be $3.06 \mathrm{ng} / \mathrm{g}$. Since the calibration function's uncertainty contributes to the predicted value, the $95 \%$ expanded uncertainty on this result is $0.24 \mathrm{ng} / \mathrm{g}$. The excellent agreement between the measured and certified values provides evidence the procedure is valid.

When statistically and substantively significant difference is detected, validation may induce a change in an assigned value, in the associated uncertainty, or in both. If no such difference is detected, then there is no penalty of increased uncertainty owing to the mere fact that a validation was carried out. 


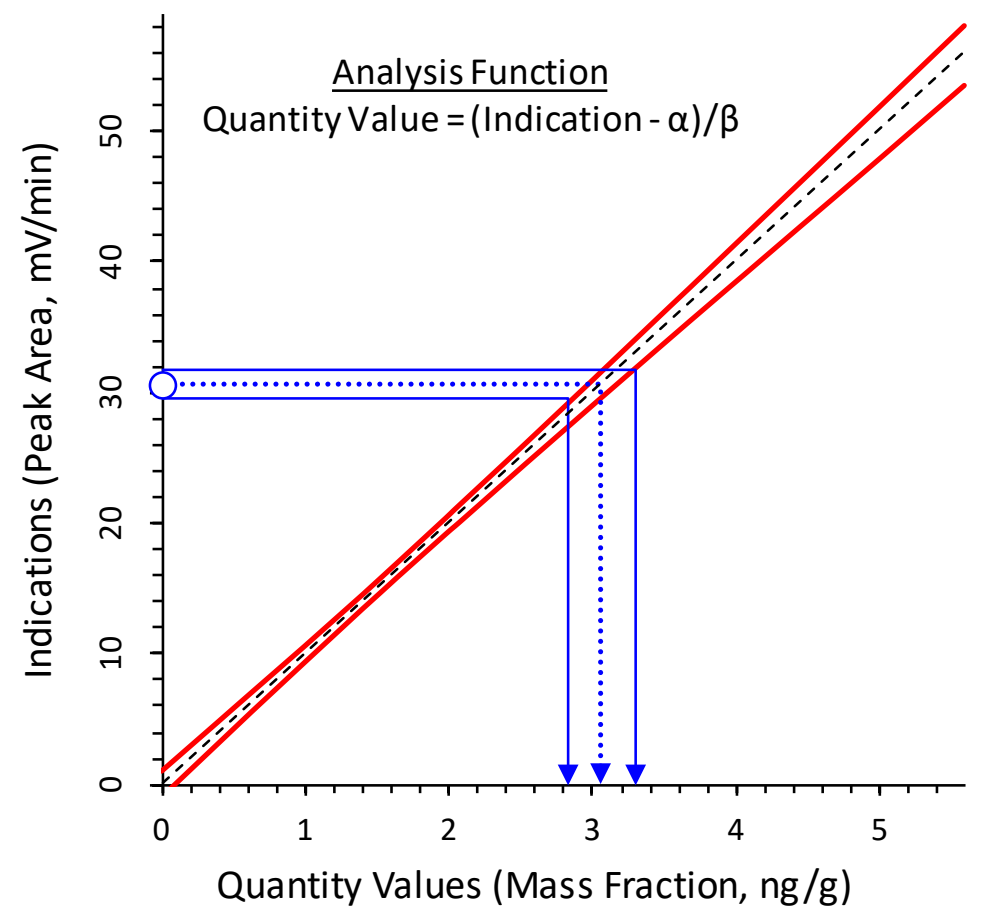

Figure 10. Quantity Values, Indications, and Analysis Function

The open circle represents a measured indication (here, a chromatographic peak area measured in $\mathrm{mV} / \mathrm{min}$ ) and its $95 \%$ expanded uncertainty. The dashed black line represents the linear calibration function: $Y=\alpha+\beta X$. The solid red curves represent the $95 \%$ uncertainty interval on the function. The dotted blue line represents the linear prediction function: $X=(Y-\alpha) / \beta$. The solid blue lines represent the propagation of the indication and calibration function uncertainties.

Note: the uncertainty contributed by the calibration function established in Figure 9 could be reduced by using more (or more strategically located) working calibration solutions.

\subsubsection{Method/Procedure/Process Independence}

The certification modes described in Section 3 require there to be fit-for-purpose agreement between two or more independent sources of information. Like fitness for purpose, "independence" is a relative concept for which there is no simple definition.

Complete analytical orthogonality (fundamentally different measurement principles) is seldom necessary even when possible. It often suffices that the most problematic steps in a process be thoroughly investigated using methods that are as different as resources permit. For instance, using different chromatographic columns, mobile phases, and detection modes may provide adequately independent evidence for the absence of co-eluting organic components in a simple calibration mixture. However, demonstrating that results are independent of the "pre-analytical" sampling, extraction, and transformation steps in a process may be the critical consideration for analytes in complex matrix materials.

Appropriate training, experience, and good judgement are keys to achieving - and recognizing - adequate independence. 


\subsubsection{Reference Measurement Procedure (RMP)}

A reference measurement procedure (RMP) is formally defined as a "measurement procedure accepted as providing measurement results fit for their intended use in assessing measurement trueness of measured quantity values obtained from other measurement procedures for quantities of the same kind, in calibration, or in characterizing reference materials" [VIM3:2.7]. In this document, an RMP is a measurement procedure that has been extensively characterized and found capable of providing accurate, metrologically traceable results for a measurand of interest. RMPs are insensitive, at least within established limits, to the sample matrix. This is particularly true of RMPs for many inorganic analytes, where one of the first steps in the analysis is to change the form of the sample to eliminate or greatly reduce the influence of the original matrix; e.g., acid digestion, thermal decomposition, or borate fusion.

Note: The development of a fit-for-purpose RMP (and the demonstration that it is indeed fit for the intended purpose) basically involves assessing precision and bias over the analytical range of interest. How to accomplish this is well beyond the scope of this document. However, a wealth of opinion and guidance is available: see references $60,61,62,63,64$ and their references.

\subsubsection{Primary Reference Measurement Procedure ("Primary Method")}

A primary RMP is a "reference measurement procedure used to obtain a measurement result without relation to a measurement standard for a quantity of the same kind" [VIM:2.8]. The key phrase here is "a quantity of the same kind," meaning that the RMP does not require calibration with a reference that delivers the same quantity. However, reference may be made to measurement standards for other quantities that influence the RMP, e.g., mass, temperature, time, etc.

For example, coulometry can be a primary method for determining amount of substance. It involves counting the number of electrons consumed in a reaction. To determine the amount of cyclohexane added to a solution of potassium bromide, two measurements are required: the applied electric current (coulombs per second) and the time (seconds) required for every molecule of cyclohexane to capture two bromine ions. Combining these measured values with the reciprocal of the Faraday constant (moles of electrons per coulomb) provides the cyclohexane amount in moles. This measurement method involves reference to standards of time and electrical current, but not to standards for the concentration of cyclohexane.

\subsubsection{Primary Direct Reference Measurement Procedures ("Primary Direct Method")}

Primary direct RMPs provide values that are directly traceable to SI units or the natural unit "count one." Examples of primary direct RMPs are: gravimetry (mass), coulometric titrimetry ( $\mathrm{mol} / \mathrm{kg}$ ), and digital polymerase chain reaction (count of targeted entities per partition volume).

\subsubsection{Primary Ratio Reference Measurement Procedures ("Primary Ratio Method")}

Primary ratio RMPs provide values that are the ratio of two values of the same quantity. Examples of primary ratio RMPs are: spectrophotometry (transmittance when the sample is in and out of the light path), internal standard ${ }^{1} \mathrm{H}$-qNMR $\left({ }^{1} \mathrm{H}\right.$-content of test sample and an internal standard of known composition), and ID-MS (signal intensity between test and isotopically labeled analyte).

\subsubsection{3. "Primary" Versus "Definitive" Versus "Always Accurate"}

It is sometimes asserted that certain "primary" or "definitive" analytical methods "irrefutably" provide accurate (i.e., true) results. ${ }^{65}$ This is an unrealistically trusting viewpoint. There may be differences between capabilities of an RMP and how it is used in a given application. " 66 "Potentially definitive" is a more appropriate non-technical term for RMPs that are expected to provide unbiased results.

The term "primary method" should only be used for methods that do not require a calibrant of the same kind as the sample. 


\subsubsection{Higher-Order Reference Measurement Procedure}

To be considered a NIST higher-order RMP, a primary direct or primary ratio RMP must be documented in detail and published in a peer-reviewed form accessible to the public. The Joint Committee for Traceability in Laboratory Medicine (JCTLM) provides a web-based database with a list of clinically relevant higher-order reference materials and reference methods. ${ }^{67,68}$

\subsection{Measurement Uncertainty}

The NIST Quality Manual for Measurement Services ${ }^{7}$ Section 3 defines measurement uncertainty as the doubt about the true value of the measurand that remains after making a measurement. It explains that measurement uncertainty is described fully and quantitatively by a probability distribution on the set of values of the measurand: at a minimum, it may be described summarily and approximately by a quantitative indication of the dispersion (scatter) of such distribution. See Reference 9 for further information.

\subsubsection{Level of Confidence}

The JCGM 100:2008 Guide to the Expression of Uncertainty in Measurement (GUM) ${ }^{10}$ Section 6.2.2 recommends "coverage probability" or "level of confidence" to describe the proportion of values of the measurand that are believed to lie within the "range of values within which the 'true value' is asserted to lie with a stated level of confidence." [ISO Guide 30:3.4] In keeping with ISO 17034:2016:3.2 usage, this document uses "level of confidence."

For scalar measurands, the GUM and NIST's Simple Guide for Evaluating and Expressing the Uncertainty of NIST Measurement Results (NIST's Simple Guide) ${ }^{9}$ refer to the "range of values" as the "coverage interval." The Simple Guide generalizes the concept to "coverage region" for more complex measurands.

\subsubsection{Stated Levels}

While specific levels of confidence are not mandated, JCGM 100:2008 GUM ${ }^{10}$ Section 4.3.4 recognizes "90, 95, or 99 percent" as levels that "one may find." The $95 \%$ level is by far the most often encountered. As NIST's Simple Guide states "In most cases, specifying a set of values of the measurand believed to include its true value with $95 \%$ probability (95\% coverage region) suffices as expression of measurement uncertainty." "Why $95 \%$ ?

- Recognition: The interval $x \pm 2 u$ is widely recognized as an approximate $95 \%$ level of confidence interval for Gaussian (normal) probability distributions of mean $x$ and standard uncertainty $u$. While the "exact" $95 \%$ interval is $x \pm 1.96 u$, the slight overestimate from rounding 1.96 up to 2 does no harm and greatly simplifies customer communications. Even for distributions markedly different from Gaussian, an interval of the form $x \pm 2 u$ often comprises approximately $95 \%$ of the total probability of the distribution.

- Practicality: The greater the asserted level of confidence, the more evidence needed to describe the tails of the probability distribution that generated the set of measurand values. Describing the central $95 \%$ of the set provides "in most cases" a practical compromise between benefit and cost.

- Limitation of the normal model: The $5 \%$ of measurand values outside the $95 \%$ interval often includes values that are much farther outside than expected for a truly Gaussian distribution.

\subsubsection{Uncertainty for Non-Certified Values}

As discussed in Section 1.2.2, NIST provides non-certified values only when the measurand identity, homogeneity, and stability have been established as fit-for-purpose, but the values' accuracy (trueness and precision) has not been confidently established. 
The uncertainty associated with a quantitative non-certified value should characterize the known variability components (typically repeatability or reproducibility precision, homogeneity, and stability). The documentation associated with the value should describe these components in enough detail for a user to have confidence in the suitability of the material for use in harmonization, method development, or quality control. However, if the true value of the measurand is not sufficiently well established to certify the value, the documentation must not imply that the true value is contained within an interval at any level of confidence.

All quantitative values delivered by an RM must be documented as non-certified regardless of how confidently the accuracy has been established. If one or more values of major interest in a material can be sufficiently well established, the RM should be upgraded to an SRM and the values certified.

\subsection{Identification Confidence}

A conventional probabilistic evaluation and expression of measurement uncertainty is not appropriate for expressing the confidence in a statement of the chemical or biological identity of the measurand in an

SRM. For identity, NIST now uses a three-level ordinal scale of confidence:

- Highest confidence: There is enough evidence to make the identification with no unresolved ambiguities or contradictions.

- Confident: There is enough evidence to make the identification, albeit with technical ambiguities that are explicable but not fully resolvable.

- No confidence: There is not enough evidence to establish the trustworthiness of the identity.

Figure 11 displays a flow diagram of the confidence assessment process. What constitutes "enough evidence" and "fully resolved" are issues to be determined by the measurement communities concerned. ${ }^{52}$

Identities can be certified only when the assessed confidence is "Highest." The "Highest confidence" assessment is reserved for certified identities.

\subsubsection{Confidence for Non-Certified Identities}

Non-certified identities are provided only when assessed as (at least) "Confident" according to explicitly stated criteria. When there are multiple measurands (e.g., for metabolomic-related RMs), identifications based on different criteria are listed in separate Tables.

All identities delivered by an RM must be documented as "Confident" regardless of how confidently the identity has been established. If one or more identities are of major interest in a material and can be established with "Highest Confidence," the RM should be upgraded to an SRM and the identities certified. 


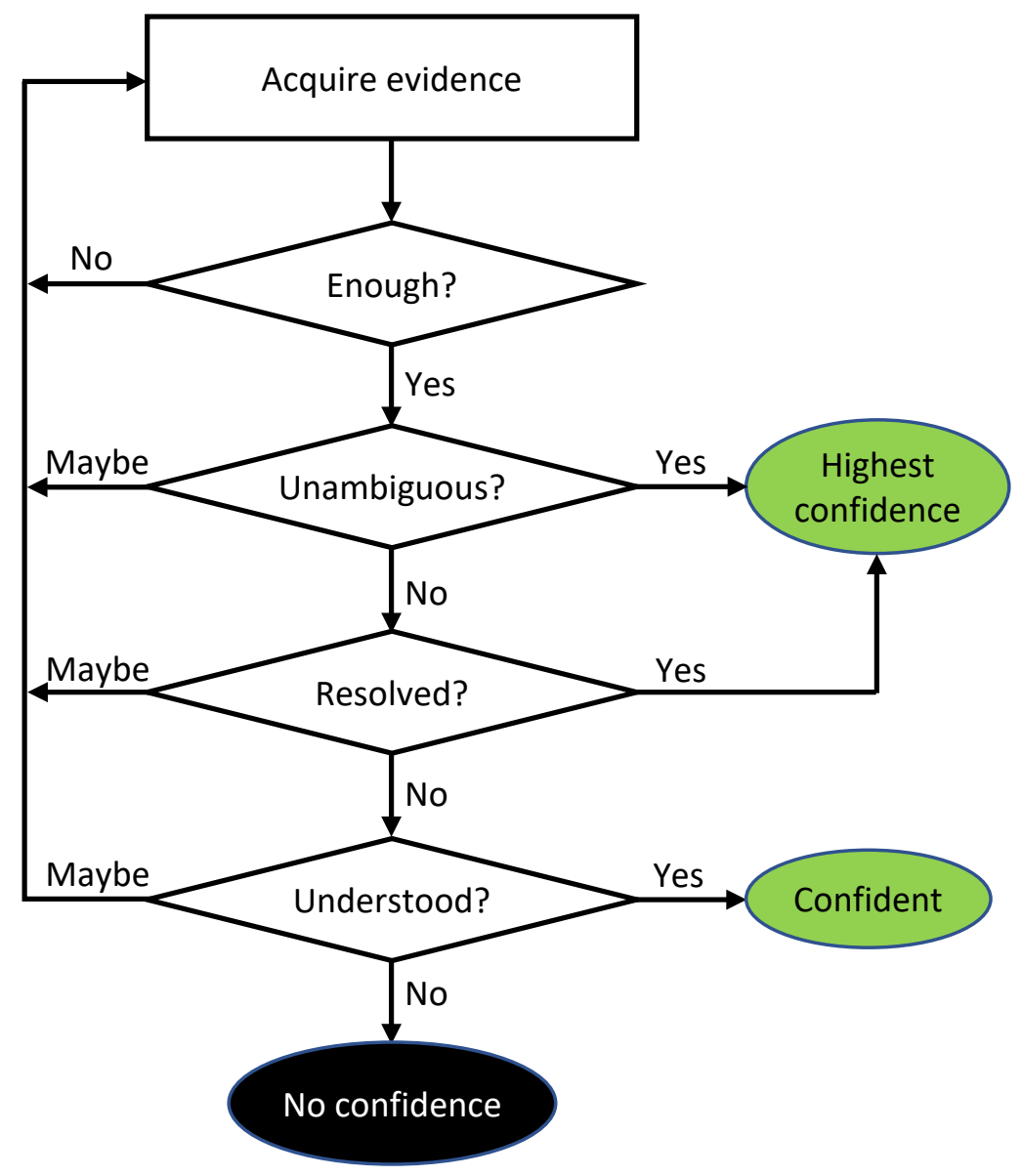

Figure 11. Process for Establishing the Confidence in an Assigned Identity

It is often difficult to establish what is "enough" evidence before quite a lot of evidence has been acquired. If some of these initial data are contradictory, further action is required - typically acquiring additional evidence to resolve the ambiguities. Highest confidence can only be achieved if all ambiguities in the initial (and any subsequent) data are fully and confidently resolved (e.g., as misinterpretations, artifacts, or under-appreciated limitations of a measurement process). If defensible root-causes for the ambiguities can be established but definitive evidence cannot be acquired, "Confident" but not "Highest confidence" identification can be established. If the root-causes of the ambiguities cannot be identified, identification cannot be established with any confidence.

Establishing chemical identity requires a fit-for-purpose determination of chemical structure.

Establishing biological identity requires fit-for-purpose comparisons with vouchered specimens that serve as holotypes for the relevant organism(s). Biological identity is increasingly based on comparisons of identifying portions of DNA.

In most cases, these determinations are not amenable to the usual "95\% level of confidence" assessment; rather confidence in the identification is based on community standards about what constitutes fit-forpurpose evidence. The assessment is intrinsically binary: there either is or there is not enough pertinent and consistent evidence to certify with "Highest confidence" that the community standards have been met. This expression of confidence typically conveys a judgement based on expert interpretation of information from multiple sources. 


\subsection{Metrological Traceability}

Metrological traceability is now formally defined as the "property of a measurement result whereby the result can be related to a reference through a documented unbroken chain of calibrations, each contributing to the measurement uncertainty" [VIM3:2.41]. The more general (and more directly appropriate for many chemical and biological measurands) term "chain of comparisons" was used in VIM2. ${ }^{43}$ However, "a comparison between two measurement standards may be viewed as a calibration if the comparison is used to check and, if necessary, correct the quantity value and measurement uncertainty attributed to one of the measurement standards [VIM3:2.42 Note 3].

The metrological traceability of the results from many types of chemical measurement is detailed in reference. ${ }^{69}$ The limits of traceability for most chemical measurements are discussed in reference 70 .

\subsection{1. "Traceable to NIST"}

"Traceable to NIST" is shorthand for "Metrologically traceable to the SI (or other higher-order reference system) through measurement results certified by NIST."

The NIST policy on traceability includes the following: ${ }^{71}$

- The provider of the result of a measurement is responsible for supporting its claim of the traceability of that result or value. This is the case whether that provider is NIST or another organization.

- The provider of a measurement result must document the measurement process and uncertainty estimates used to establish the claim and provide a description of the chain of calibrations that were used to establish a connection to a specified reference.

- The user of the result of a measurement is responsible for assessing the validity of a claim of traceability.

\subsubsection{Traceability Chain}

A metrological traceability chain is the "sequence of measurement standards and calibrations that is used to relate a measurement result to a reference" [VIM3:2.42]. Descriptions of a traceability chain must include the calculations and uncertainty estimates used to establish the relationship.

The following entries present generic graphical representations of the traceability chains for results traceable to reference systems through NIST SRIs, PSs, and SRMs. Figure 12 presents the simple chain appropriate to SRI results and Figure 13 presents the chain for results that trace through SRM calibrants.

In the absence of a suitable calibration CRM, metrological traceability can be established by calibrating with a matrix material that delivers a certified value for the measurand of interest (see Section 2.13.5). However, the traceability chain for a matrix material has at least one more link than that of a calibration material and so will not provide the smallest possible measurement uncertainty. Calibrating with a matrix material may also lead to biased results unless the measurement procedures used are known to be relatively insensitive to the composition of the matrix. Figure 14 presents the partial traceability chain when results from a measurement procedure are calibrated using a matrix SRM. 


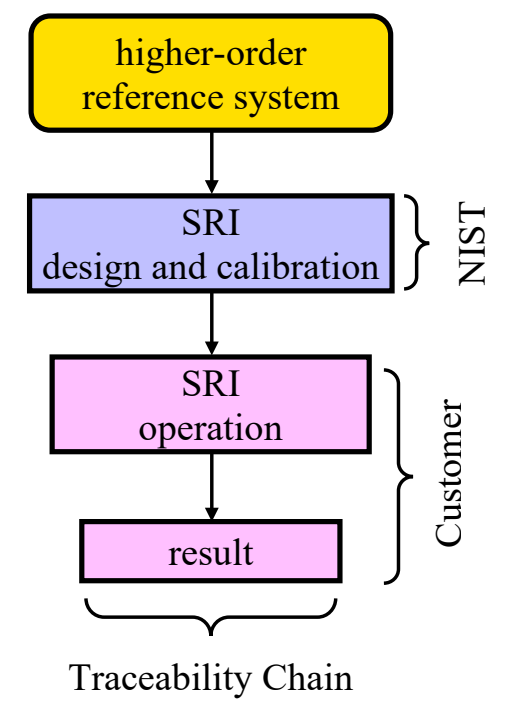

Figure 12. Metrological Traceability of Results from a NIST Standard Reference Instrument (SRI). Arrows represent links in the calibration chain; the direction of the arrows indicates the inheritance path. NIST establishes that measurement results provided by the SRI are traceable to the higher-order reference system (typically the SI) when the reference instrument is maintained and used as directed by NIST. It is the customer's responsibility to establish traceability by documenting that the SRI is properly operated and maintained. 


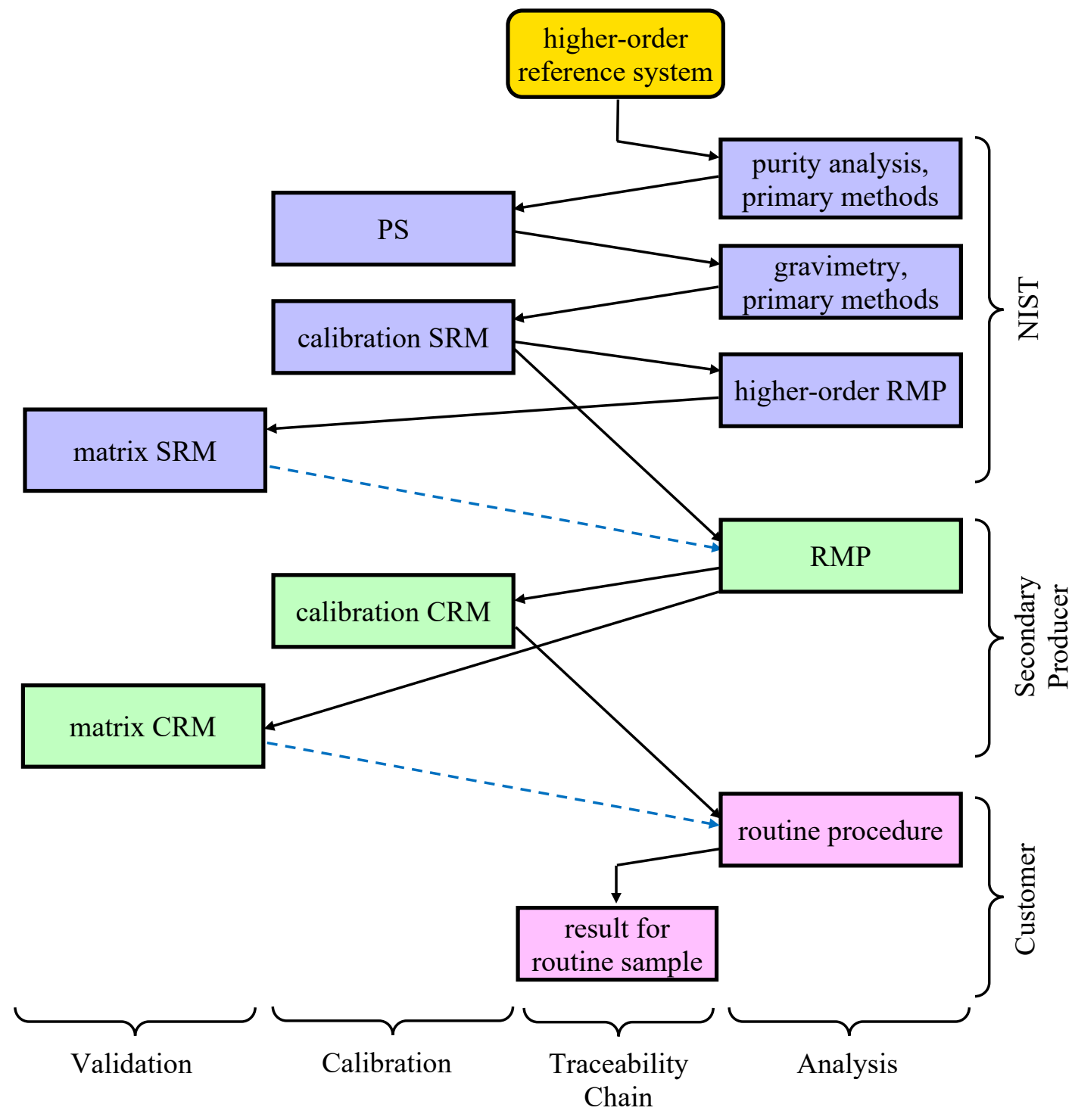

Figure 13. Metrological Traceability for Results Using NIST's Reference Materials

Solid arrows represent links in the calibration chain; dashed arrows represent validations. The direction of the arrows indicates the inheritance path. NIST establishes that the certified values delivered by its Standard Reference Materials (SRMs) are traceable to the higher-order reference system (typically the SI) when properly stored and used. However, SRMs are intended to be used by secondary standards producers and other expert customers to calibrate and validate RMPs that they use to value-assign customer-oriented calibrant and validation materials. It is the secondary producer's responsibility to establish the traceability of the assigned values. It is the customer's responsibility to establish the traceability of their measurement results by documenting that their measurement procedure is appropriately calibrated and validated. 


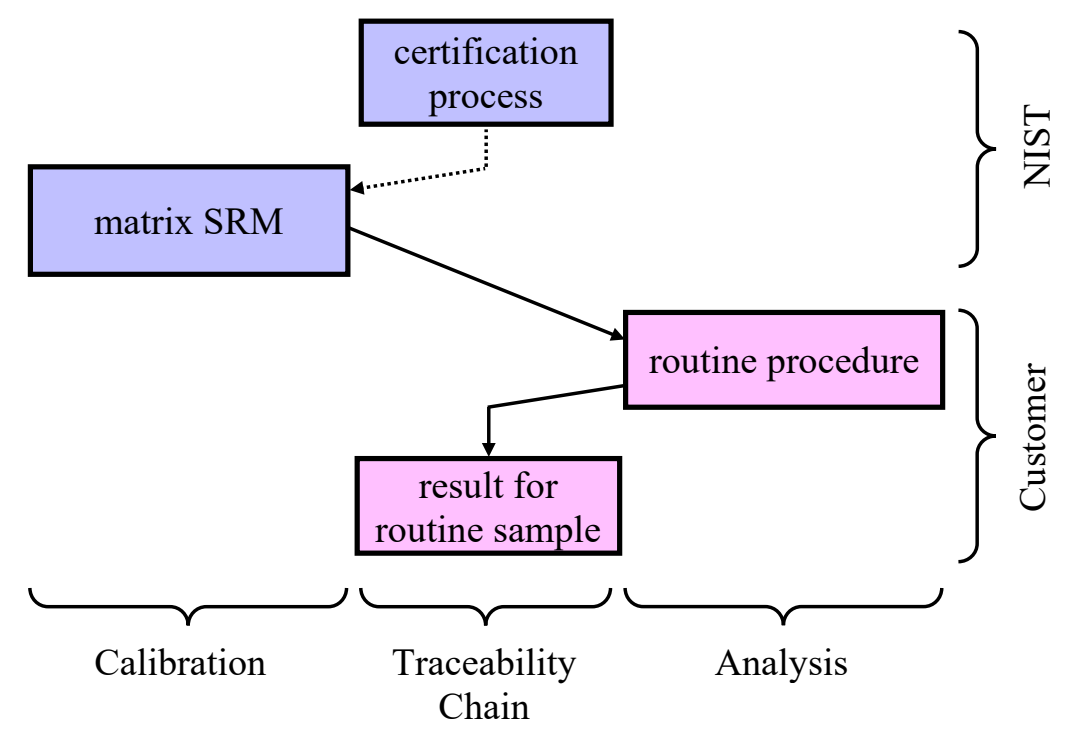

Figure 14. Metrological Traceability for Results When a Matrix Material is Used as a Calibrant

Solid arrows represent links in the calibration chain; the dotted arrow represents a contingent linkage. The direction of the arrows indicates the inheritance path. When a measurement procedure is calibrated using a matrix SRM, the results produced for a routine sample are metrologically traceable to the SRM. It is the customer's responsibility to determine if the measurand realized by their procedure is the same as that realized by the certification process. If identical, the traceability of the result can be extended to the SRM's reference system. If not identical, the result of the measurement may be subject to bias due to the measurand mismatch.

\subsubsection{Commutability}

CRM commutability is formally defined as the "property of a reference material, demonstrated by the closeness of agreement between the relation among the measurement results for a stated quantity in this material, obtained according to two given measurement procedures, and the relation obtained among the measurement results for other specified materials" [VIM3:5.15]. More generally, it is the "property of a reference material, demonstrated by the equivalence of the mathematical relationships among the results of different measurement procedures for a [reference material] and for representative samples of the type intended to be measured" [ISO Guide 30:2015:2.20]. However, the spirit of the concept is more directly captured by a definition from clinical literature: “... a property of a reference material such that values measured for that reference material and for representative clinical samples have the same relationship between two, or more, measurement procedures for the same measurand."72 That is, for clinical CRMs commutability refers to how well the CRM mimics the properties of clinical samples when being analyzed. $^{73}$

Unfortunately, these definitions fail to recognize that commutability is a property of interactions between reference materials and specific measurement procedures, not the materials in isolation. Different procedures may be influenced by different interferences. Materials that are realistically representative of typical sample materials for some procedures may not be for others. Establishing the commutability of a CRM among any fixed set of procedures does not ensure that it will be suitable for the next procedure. It is impossible to design a CRM that is inherently commutable; however, understanding the operational principles and potential interferences of the measurement procedures for which the CRM will be used increases the likelihood that the CRM will be fit for purpose. 
Most NIST matrix SRMs are intended to help validate the RMPs of secondary standards producers and other users who prepare metrologically traceable reference materials suitable for use with their own procedure(s). Therefore, the certification modes described in Section 3 are intended to ensure that NIST's materials are commutable among RMPs that are relatively insensitive to differences in sample matrix. Figure 15 displays the two commutability domains in the traceability chain.

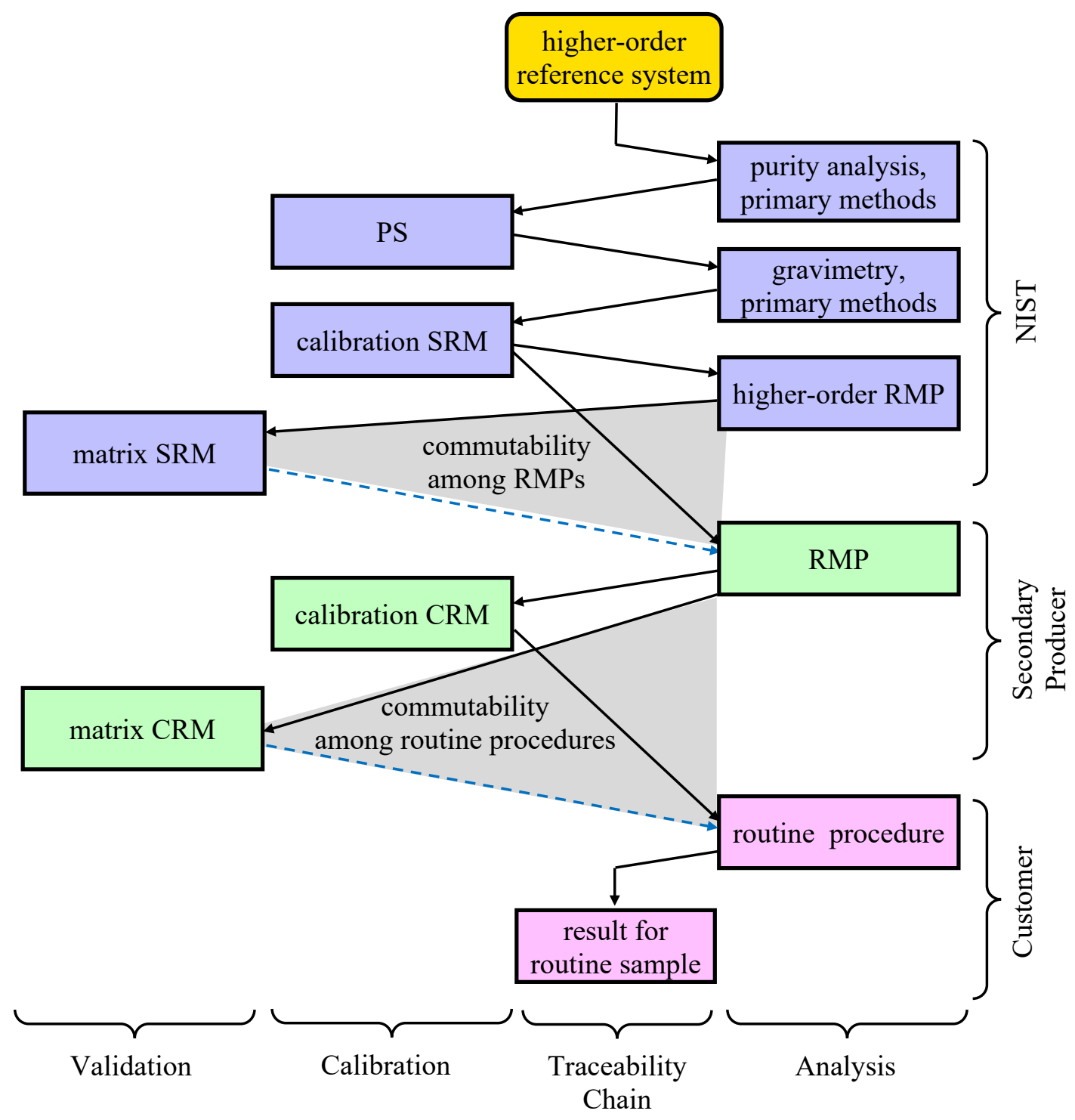

Figure 15. Commutability Responsibilities for Producers and Users of Matrix CRMs

Solid arrows represent links in the calibration chain; dashed arrows represent validations. The direction of the arrows indicates the inheritance path. SRMs are designed to be commutable among reference measurement procedures (RMPs), that is procedures that are relatively insensitive to non-target components of the matrix. They are not designed to be commutable among routine measurement procedures. Evaluating the commutability of CRMs designed for use with routine procedures is the responsibility of the procedures' developers and users. 
Some matrix CRMs are used by manufacturers to verify calibration of their routine measurement procedure. In this case, it is the manufacturer's responsibility to ensure that the CRM is suitable for use as a trueness control.

\subsubsection{Standardization and Harmonization}

In a metrological context, standardization is used for the process of modifying measurement procedures to provide true values. Harmonization is used when the goal is to bring procedures into agreement even when truth is unknown. SRMs are intended to support standardization efforts. RMs are intended to support harmonization efforts.

Note: Calibration to a non-certified value, whether delivered by an RM or CRM, establishes metrological traceability only to that value and not to any higher-order reference system. The material that delivers a non-certified value provides a useful reference system for that measurand during the measurand's period of validity and the material's availability.

\subsubsection{Trackability}

While "trackability" is sometimes misused as a synonym for (metrological) traceability, the colloquial sense of "ability to be tracked" does have metrological utility as the chronological history of materials and their associated documentation: who has it, where is it, and under what conditions is it stored. The current phrase "farm-to-fork traceability" is an example of the colloquial usage. ${ }^{74}$

\subsection{Reference Material}

The VIM3 and ISO Guide 30:2015 definitions for the general class of reference materials are congruent:

- material, sufficiently homogeneous and stable with reference to specified properties, which has been established to be fit for its intended use in measurement or in examination of nominal properties [VIM3:5.13].

- material, sufficiently homogeneous and stable with respect to one or more specified properties, which has been established to be fit for its intended use in a measurement process [ISO Guide 30:2015:2.1].

Note: This document uses the term "measurement" for the determination of all properties, whether quantitative, qualitative (nominal), or somewhere in between (ordinal).

\subsubsection{Candidate Reference Material}

A candidate reference material is a "material, intended to be produced as a reference material [ISO Guide 30:2015:2.3]. However, not all that is intended necessarily comes to pass: materials should not be explicitly identified in public-facing documents or presentations unnecessarily.

\subsubsection{Certified Reference Material (CRM)}

The VIM and ISO Guide 30:2015 definitions are congruent, but differ in their emphasis:

- reference material, accompanied by documentation issued by an authoritative body and providing one or more specified property values with associated uncertainties and traceabilities, using valid procedures [VIM3:5.14]

- reference material characterized by a metrologically valid procedure for one or more specified properties, accompanied by a [reference material] certificate that provides the value of the specified property, its associated uncertainty, and a statement of metrological traceability [ISO Guide 30:2015:2.2]

This document follows the Guide 30:2015 definition with its emphasis on the certificate. 


\subsubsection{Matrix Reference Material}

ISO Guide 30:2015:2.4 states that a matrix reference material is "characteristic of a real sample" with Notes that they:

- $\quad$ are intended to be used in conjunction with the analysis of real samples of the same or a similar matrix,

- may be obtained directly from biological, environmental, or industrial sources,

- may be prepared by spiking the component(s) of interest into an existing material,

- $\quad$ are not chemical substances dissolved in pure solvent.

Since what constitutes a "real sample" is contingent on the material's intended purpose, these Notes are incomplete. Relative to their purpose(s), NIST matrix materials are as like "real samples" as is practical given the need for homogeneity, stability, and production of suitable numbers of units. The concept of real samples applies equally to "natural" samples and to created materials like alloys if the measurand they deliver is fit for the intended purpose(s).

When spiking is used to prepare a material, care must be taken to ensure that the analysis methods to be used are valid for both the naturally occurring and spiked measurand.

Note: The term "natural-matrix material" is sometimes used to distinguish between materials with "complex" (e.g., tissues, soils, alloys) and "simple" (e.g., hexane, acidified water, pure metals) matrices. Given that the distinction between simple and complex depends on the intended purpose, this document does not distinguish between natural (native) and unnatural (purpose-created) matrices.

\subsubsection{Primary Standard}

In this document, chemical and biological PSs are nominally single-component solids or liquids or simple mixtures of gases that have been value-assigned with a fit-for-purpose purity and fit-for-purpose measurement uncertainty. Although some PSs are assigned nominal property values, typically primary component identity, these characterizations are substantiated through purity assessment. The purity assessment process used to assign the value and its uncertainty provides linkage to a higher-order reference system.

PSs need not be of the highest feasible purity nor exhaustively characterized, just of high enough purity and characterized well enough to be fit for their intended purpose. ${ }^{75,76}$ Production of single-element PSs that can be used to produce calibration solutions that enable SI-traceability with fit-for-purpose $95 \%$ expanded relative uncertainties of $0.2 \%$ can typically be accomplished using relatively routine and inexpensive multi-element methods. However, the PS1 qNMR standard was designed to be the ultimate link between the SI and qNMR purity results for virtually all hydrogen-containing small organic compounds. ${ }^{102}$ To be fit for this purpose, the material was exhaustively (and expensively) characterized with all relevant analytical methods and a novel mathematical approach developed to combine the resulting information. The PS1 benzoic acid mass fraction is certified to be $999.92 \mathrm{mg} / \mathrm{g}$ with a $95 \%$ level-of-confidence expanded uncertainty of $0.05 \mathrm{mg} / \mathrm{g}(0.005 \%$ relative $)$.

\subsubsection{Validators and Calibrants}

For each measurement procedure, a CRM can only be used either as a calibration standard or in a validation role. Re-analyzing a CRM that was used to calibrate a procedure can only "validate" whether the procedure is stable and whether the data analysis is working.

A calibration CRM (calibrant) is a "reference material used for calibration of equipment or a measurement procedure" [ISO Guide 30:2015:2.21]. Most SRMs intended for use in calibration, especially when using NIST measurement procedures, are nominally "pure" compounds or simple 
mixtures of one to a few compounds. The intended purpose of calibrant SRMs is to provide higher-order reference system metrological traceability to the results of methods that have been calibrated with them. That means the uncertainty of the assigned value must be propagated, and the measurand certified must match the measurand of the measurement procedure being calibrated.

Matrix CRMs may be a defensible choice for calibration of a measurement procedure. This approach is frequently used for routine measurements, especially with measurement procedures that require calibrants to be as similar to samples as possible. Matrix CRMs are used at NIST on occasions when an independent or confirming analysis is needed, no NIST RMP has been developed, and resources are limited. As an example, XRF can be calibrated for mass fractions of elements in food and dietary supplement materials. Because NIST has not developed a sample preparation approach to overcome matrix composition biases for these complex materials suitable for use with XRF, it is expedient to calibrate the spectrometer using a set of carefully selected matrix SRMs of other types, with both calibrants and samples made into pressed briquettes. Establishing uncertainty estimates and traceability statements for these results may be less rigorous than for higher-order RMPs, but they are fit for their intended purpose.

A validation CRM (validator) is simply one intended for use in conformity (lack of bias) assessment of measurement procedures. Most validators have relatively complex matrices because laboratories need to convince clients and third party auditors that validation has been accomplished with a CRM very close in composition to the organization's products. Sometimes NIST has a similar need when an RMP must be shown to be free of bias.

The concepts of calibration and validation are blended in VIM3:2.42, Note 3: "A comparison between two measurement standards may be viewed as a calibration if the comparison is used to check and, if necessary, correct the quantity value and measurement uncertainty attributed to one of the measurement standards." Here, one reference material is the calibrant and one is a validator. The verb "to check" means to validate, and "to correct" is to alter the calibration. Even when a bias is detected, it is preferable to improve the method and to identify and eliminate the bias rather than to apply corrections for selected materials. ${ }^{77}$ Corrections come with additional uncertainty because of assumptions that must be made and complicate both the measurand definition and traceability link(s).

Because there may be only one CRM that provides a composition within the scope of measurement, an analyst may be faced with the need to decide whether to calibrate with the CRM or to validate with it. If the measurement procedure requires use of a matrix CRM as a calibrant to avoid sample matrix bias, there will be no direct way to validate the method. In this situation, the analyst may wish to consider alternative calibration models that enable calibration using a PS, such as the method of standard additions. ${ }^{78}$ Standard addition methods exploit changes in instrumental response with added analyte to estimate the quantity of the analyte originally in the sample. When the added analyte does not significantly alter the sample matrix and the relationship between the quantity and the instrument response is well-defined, this can provide bias-free use of a calibrant CRM thus freeing the matrixmatched CRM for use in validation. ISO/IEC 17025:2017 General requirements for the competence of testing and calibration laboratories ${ }^{3}$ Section 7.2.2.1 suggests several alternative approaches for validating a calibrated method.

\subsection{Secondary Standard Producer}

For the purposes of this document, a secondary standard producer is defined as any (suitably accredited) CRM-producing organization that uses NIST (or another NMI) CRMs or higher-order RMPs to establish the metrological validity and traceability of their products. Secondary standard producers thus leverage the commercial and scientific impacts of NMI products. 


\subsection{Resellers and NIST-Licensed Distributors}

Beginning in 2014, NIST has licensed selected distributors to resell our reference materials. These NISTLicensed Distributors are third party-accredited as CRM producers of products of similar kind to the NIST materials that they resell. This provides confidence to NIST and our customers that these distributors have the expertise and facilities to properly handle and store NIST materials.

The non-exclusive license enables a distributor to purchase materials from NIST and resell them into the distributor's market with full NIST support of the product and its integrity. To the benefit of both NIST and our customers, these distributors can make NIST materials more readily available, especially to customers in locations that are difficult or expensive to ship to. Although the distributor may add additional label information, the material is distributed in its original packaging, with its NIST label and current NIST documentation. Licensed Distributors are authorized to report their status in their catalogs and other promotions.

There are organizations and individuals who are not NIST-Licensed Distributors that choose to resell NIST materials. It is the end user's responsibility to determine whether they are purchasing from a Licensed Distributor and, if not, whether the purchased material has been stored and shipped appropriately. 


\section{Certification Modes}

The following sections describe the approaches or "modes" NIST currently uses or anticipates using for certifying values for its PS, SRM, RGM, and NTRM tools. The descriptions present minimal requirements along with illustrative examples. These descriptions replace those described in previous documentation. ${ }^{1}$

Determining which approach is "best" for a given certification depends upon the resources (including people, time, appropriate equipment, and money) required to certify the value as well as the tool's purpose. Having a clearly stated need is the first and most important step in deciding how to efficiently provide a valid solution. This decision is critical when beginning projects but does not preclude revising the scope when circumstances change.

Since there is considerable overlap among some of the characterization modes, differing more by how analytical results are used to assign values rather than how they are obtained, the measurement procedures used to certify a given value are sometimes a hybrid of two (or more) of these modes. NIST CRMs that deliver multiple certified values need not (and often do not) use the same procedures for all measurands.

\subsection{One Higher-Order Reference Measurement Procedure at NIST}

Certification at NIST using a single higher-order reference measurement procedure (higher-order RMP) for the measurand of interest requires that:

- the RMP be formally documented in a publicly accessible form,

- all potentially significant sources of uncertainty have been evaluated for the measurand of interest, and

- the results of the RMP are independently confirmed.

Confirmation can be achieved by:

- comparison of results from suitably independent measurements performed by NIST, by one or more expert collaborating laboratories, in an ILS, or by demonstrating the performance of the RMP through analysis of an existing CRM for the same measurand and similar composition.

The required level of agreement between the higher-order RMP and validation information must be predetermined. ${ }^{66,79}$

This mode is appropriate for materials that deliver a measurand that can be accurately determined by the higher-order RMP, at a level known to be within the analytical range of the procedure, and with an uncertainty that is fit-for-purpose. Results from that determination are frequently confirmed using one or more relatively independent (if of less high-order) measurement procedures. Examples include:

- SRM 660c Line Position and Line Shape Standard for Powder Diffraction (Lanthanum Hexaboride Powder) ${ }^{80,81}$

This SRM is certified with respect to lattice parameter and, as such, it is used to calibrate equipment with regard to profile position. It has been prepared to display a minimal level of microstructure-induced profile broadening; its primary function is to determine the peak shapes associated with the diffraction equipment itself. The use of SI-traceable emission spectra provides the certified lattice constants with a linkage to the fundamental unit of length, the meter. ${ }^{82}$ The angular scale of the measured peaks is provided via a high-precision optical angular encoder. These measured angles are also affected by an instrumental aberration function, which is determined from the known instrument geometry and the use of the Fundamental Parameters Approach. ${ }^{83,84}$ Thus, all components of the measurement are directly traceable to first principles.

- SRM 931h Liquid Absorbance Standard for Ultraviolet and Visible Spectrophotometry ${ }^{85,86}$ This SRM is intended for critical evaluation of daily working standards used in spectrophotometry and for use as an accuracy check of the photometric scale of 
spectrophotometers that provide a narrow effective spectral bandpass. Each SRM 931h unit consists of three cobalt-nickel liquid filter solutions at different concentrations and a blank solution. The certified net absorbances, corrected for the absorbance due to the blank solution, are determined using primary ratio measurements made at NIST. Results are verified through evaluation of selected sets of glass absorbance filter SRMs.

- SRM 1720 Northern Continental Air ${ }^{87,88,89,90}$

Single-analyte primary standard (PS) gas mixture suites that span concentrations of analytical interest are produced from well-characterized starting materials using the primary direct method of gravimetric preparation. SRMs, such as the natural-background air SRM 1720, are valueassigned relative to the NIST gas PSs (a primary ratio method). The assigned values were verified by comparison to results provided by the Earth System Research Laboratories of the National Oceanic and Atmospheric Administration (NOAA), Boulder, Colorado, a designated institute of the World Meteorological Organization. SRM 1720 is intended for calibrating instruments used for measurement of ambient carbon dioxide and other trace gases.

- SRM 1934 Fluorescent Dyes for Quantitative Flow Cytometry (Visible Spectral Range) ${ }^{91,92}$ This SRM is intended for use by stakeholders in the quantitative flow cytometry community to assign fluorescence intensity values to calibration microspheres for fluorescence channels of flow cytometers in the visible spectral range. It is also used internally by NIST to make similar assignments for members of the Flow Cytometry Quantitation Consortium. The SRM consists of fluorescent solutions certified for fluorophore concentration and purity. Primary ratio qNMR spectrometry was used as a primary method to determine the mass purity of each fluorophore. The purity was independently verified by analysis of impurities using a variety of other analytical techniques.

- SRM 2374 DNA Sequence Library for External RNA Controls ${ }^{93}$ This External RNA Controls Consortium (ERCC)-sponsored SRM delivers 96 unique doublestranded DNA nucleotide sequences. Use of these materials as external controls supports confidence in gene expression assays by providing quantitative assessment of the technical performance of gene expression measurements. For each control, complete Sanger sequencing of both strands was performed on replicate samples. The resulting data were independently handcurated and evaluated for both sequence and confidence estimation by two analysts. Discrepant results were resolved using two independent, ultra-high throughput, short-read sequencing experiments performed on two different platforms.

- SRM 2924 C-Reactive Protein Solution 94,95

This SRM is intended for use in calibrating procedures and devices for the determination of C-reactive protein (CRP), an inflammation marker in human serum. The molar concentration of CRP was determined by primary ratio amino acid analysis using isotope dilution liquid chromatography tandem mass spectrometry (ID-LC-MS/MS) and verified using a contemporaneously developed CRM produced by the National Metrology Institute of Japan.

- SRM 2973 Vitamin D Metabolites in Frozen Human Serum (High Level) ${ }^{\text {96,97,98 }}$

SRM 2973 is intended to be used as an accuracy control in the critical evaluation of methods for determining the concentration of vitamin D metabolites in human serum. The concentration of 25-hydroxyvitamin $\mathrm{D}_{3}\left(25(\mathrm{OH}) \mathrm{D}_{3}\right)$ in serum is an indicator of vitamin $\mathrm{D}$ status; that of 24R,25-dihydroxyvitamin $\mathrm{D}_{3}\left(24 \mathrm{R}, 25(\mathrm{OH})_{2} \mathrm{D}_{3}\right)$ is a catabolism marker and an indicator of kidney disease. ${ }^{99}$ Certified values for $25(\mathrm{OH}) \mathrm{D}_{3}$ and $24 \mathrm{R}, 25(\mathrm{OH})_{2} \mathrm{D}_{3}$ are based on results from ID-LC-MS/MS procedures recognized as higher-order reference measurement procedures by the JCTLM. SRM 2973 was distributed as a blinded study material in a 2014 ILS; results confirmed the accuracy of the NIST results and the suitability of the material as an analytical accuracy control. 
- SRM 3037 Arsenous Acid (AsIII) Standard Solution ${ }^{100}$

SRM 3037 is a primary calibration standard for the quantitative determination of arsenous acid, a water-soluble arsenic species. An acidified aqueous solution was gravimetrically prepared from arsenic trioxide to contain a known mass fraction of arsenous acid. The mass fraction of arsenic III in the solution was determined using gravimetric titrimetry. The titrant was determined using a primary ratio method, relative to SRM 83d Arsenic Trioxide (Reductometric). SRM 83d was certified using a primary direct coulometric titrimetry RMP. Impurity arsenic species were measured by ion chromatography-inductively coupled plasma mass spectrometry (IC/ICP-MS) and liquid chromatography-inductively coupled plasma mass spectrometry (LC/ICP-MS).

\subsection{Two or More Independent Reference Measurement Procedures at NIST}

Value-assignment can be based on NIST results from two or more relatively independent, NISTcharacterized reference measurement procedures (RMPs, but not necessarily higher-order RMPs). ${ }^{101}$ While RMPs rarely have completely different sources of uncertainty, similarities between sample preparation and instrument calibration methods must be minimized. Ideally, the RMPs should be calibrated using completely independent source materials; when only a single suitable source material is available, the RMPs should be calibrated using independently sampled and prepared subsamples of that material. The RMPs must provide analytical responses using different physical, spectroscopic, or chemical phenomena. The measurement uncertainty associated with the combined result includes a component addressing any persistent between-method differences.

This mode is appropriate whenever NIST has implemented and critically evaluated two or more suitable RMPs for the measurand. Examples include:

- $\quad$ NIST PS1 Primary Standard for Quantitative NMR (Benzoic Acid) ${ }^{102}$

High-purity benzoic acid was value-assigned using primary direct coulometric acidimetry and primary ratio qNMR spectrometry. Confirmatory evidence was provided by the close agreement with a mass-balance estimate based on the amount of water as determined by Karl Fischer titration and non-volatiles by ash analysis, and the absence of organic solvents and structurally related impurities from diverse analytical techniques. This material is intended to provide the ultimate metrological traceability link between the SI and chemicals inherently determinable by qNMR.

- Sodium in SRM 956d Electrolytes in Frozen Human Serum ${ }^{103,104}$

This SRM is intended for use in validating clinical analysis procedures for the determination of electrolytes in human serum or plasma. The certified concentrations of sodium are based on measurements made at NIST using inductively coupled plasma-optical emission spectrometry (ICP-OES) and ion-selective electrode (ISE) potentiometry.

- SRM 1003c Glass Beads - Particle Size Distribution ${ }^{105}$

This SRM is intended for use in the evaluation and calibration of equipment used to measure particle size distributions in the $20 \mu \mathrm{m}$ to $50 \mu \mathrm{m}$ diameter range. The cumulative mass distribution as a function of particle diameter was certified from $18.8 \mu \mathrm{m}$ to $43.3 \mu \mathrm{m}$ as the average of results from laser light scattering (LLS) and electrical sensing zone (ESZ) RMPs. The consensus values were verified using scanning electron microscopy (SEM) analysis of several thousand beads.

- SRMs 1649b Urban Dust and 1974c Organics in Mussel Tissue (Mytilus edulis) $)^{17,106,107}$ These SRMs are intended for use in evaluating analytical methods for the determination of polycyclic aromatic hydrocarbons (PAHs) and other organic and inorganic measurands in air particulates and biological matrices. Certified values for numerous PAHs were assigned based on NIST measurements using four different analytical techniques based on gas chromatography/mass spectrometry (GC/MS) on different stationary phases and on reversed- 
phase liquid chromatography (RPLC) with fluorescence detection and different extraction and cleanup procedures.

- SRM 2372a Human DNA Quantitation Standard ${ }^{108}$

SRM 2372a is intended for use in value-assigning secondary human DNA quantification materials used as daily control materials by human-identification communities. The number concentration of human DNA haploid genomes in aqueous buffer solutions was determined using primary direct chamber and droplet digital polymerase chain reaction (cdPCR and ddPCR) techniques and primary direct measurements of ddPCR droplet volume. The mass concentration of the DNA in these solutions was determined from these measurement results using critically evaluated literature values for nucleotide molar mass and expected number of nucleotides per haploid genome. Variability among multiple independent assays of number concentration is the dominant component of the assigned measurement uncertainty.

- SRM 2373 Genomic DNA Standards for HER2 Measurements ${ }^{109,110}$

SRM 2373 is a cancer biomarker standard designed to help achieve measurement assurance for precision medicine. HER2 (official gene name ERBB2) is a member of the epidermal growth factor receptor family and is frequently amplified (increased copy numbers) in cancer.

Measurement of the amplification of HER2 gene copy number is important for cancer diagnostics and treatment. Five human breast cancer cell lines with different amounts of amplification were used to prepare high-quality DNA. Determination of the amount of DNA amplification relative to a suite of four reference genes was accomplished using two orthogonal measurement methods, droplet digital PCR (ddPCR) and quantitative PCR (qPCR), using assays targeting different regions of the genes. The qPCR and ddPCR assays were validated using SRM 2372a and a synthetic plasmid containing HER2 DNA sequences.

- SRM 2391d PCR-Based DNA Profiling Standard ${ }^{111}$

This SRM is intended for use in the standardization of forensic and paternity quality assurance procedures for polymerase chain reaction (PCR)-based genetic testing. Human autosomal short tandem repeat (STR) genotypes were certified for 35 autosomal and $7 \mathrm{X}$-chromosome genetic loci in four unrelated single-source samples and in one mixed-source sample and $28 \mathrm{Y}$ chromosome loci for the three samples that contain male DNA. The genotypes were evaluated at NIST by electrophoretic base pair (bp) size match to sequenced alleles, Next-Generation Sequencing (NGS), and the concordance of results from all commercially available PCR multiplex STR-typing kits of forensic interest at the time of issue.

- SRM 2971 24R,25-Dihydroxyvitamin $D_{3}$ Calibration Solution 112

This SRM is intended as a primary calibrant for instruments and techniques used to determine $24 \mathrm{R}, 25(\mathrm{OH})_{2} \mathrm{D}_{3}$. The mass fraction of $24 \mathrm{R}, 25(\mathrm{OH})_{2} \mathrm{D}_{3}$ was value assigned by gravimetric preparation using materials of defined purity and measurement results from an ID-LC-MS/MS RMP.

- SRM 3949 Folate Vitamers in Frozen Human Serum ${ }^{113,114,115}$

This SRM is intended for use in validating methods for determining folate vitamers in human serum. Folic acid, also called vitamin $\mathrm{B}_{9}$, is used to treat anemia caused by folate deficiency and as a supplement by women during pregnancy to reduce the risk of neural tube defects in the baby. ${ }^{116}$ A unit of SRM 3949 consists of three vials each having different levels of the vitamers: low, medium, and high. Folic acid concentrations in the three sera were certified using NIST results from two suitably independent ID-LC-MS/MS methods and ID-LC-MS/MS results provided by the Centers for Disease Control and Prevention (CDC). 


\subsection{One Reference Measurement Procedure at NIST and Expert Collaborators or Interlaboratory Studies (ILS)}

Value-assignment based on NIST results from one well-characterized RMP (not necessarily a higherorder RMP) combined with results from:

- one or more expert collaborating laboratories using well-characterized RMPs substantially different from the RMP used by NIST. Reporting requirements for the expert collaborators' results are specified in the certification plan. The collaborators' report contains enough detail about the method, calibrants, and control materials to permit NIST to evaluate the fitness of the measurements.

- a suitably large ILS documented and administered by NIST or an experienced organization in collaboration with NIST. Results from the study are provided with enough detail to enable independent assessment of the consensus value.

This mode is appropriate when NIST has implemented and critically evaluated one suitable RMP for the measurand and there are suitable expert collaborators that can supply results from independent RMPs or consensus results from one or more suitably large ILSs. Examples include:

- SRM 968f Fat-Soluble Vitamins in Frozen Human Serum ${ }^{117}$

This SRM is intended for validating methods for determining fat-soluble vitamins in human serum and plasma and for qualifying control materials produced in-house and analyzed using those methods. Values for three fat-soluble vitamins were certified from NIST results provided by a well-characterized and validated liquid chromatographic RMP and from three NISTcoordinated ILSs, each with more than 20 participants.

- SRM 1635a Trace Elements in Coal (Subbituminous) ${ }^{118}$

This SRM is intended for the evaluation of techniques used in the analysis of coals and materials of a similar matrix. The certified mass fraction value for sulfur is based on measurements made at NIST using microwave-induced combustion and isotope dilution via sector-field inductively coupled plasma mass spectrometry (ICP-MS) and results from several published RMPs provided by participants in the CANSPEX 2008-3 ILS, conducted by Quality Associates International, Ltd.

- SRM 2034 Holmium Oxide Solution Wavelength Standard (240 $\mathrm{nm}$ to $650 \mathrm{~nm}){ }^{119,120,121}$ This SRM is intended for use in the verification and calibration of the wavelength scale of ultraviolet and visible absorption spectrophotometers having nominal spectral bandwidths not exceeding $3 \mathrm{~nm}$. The locations of transmittance minima for 14 absorption bands of a holmium oxide solution, containing a mass concentration of $40 \mathrm{~g} / \mathrm{L}$ in $10 \%$ (volume fraction) perchloric acid in distilled water, were certified using primary ratio results from multiple determinations by 15 independently calibrated research spectrophotometers. While these measurements were for a single preparation of the solution, they were coupled with comparisons of many preparations of the material evaluated on a single instrument at NIST.

- SRM 2894 Ethanol-Water Solution (Nominal Mass Fraction $0.1 \%{ }^{122}$

SRM 2894 is intended for use in the calibration of instruments and techniques used for the determination of ethanol in blood. The mass fractions of ethanol in water in this and several related SRMs were certified based on gravimetric preparation and purity measurements made at NIST, titrimetric determinations made at the National Measurement Institute of South Africa, and exact matching isotope dilution-gas chromatographic/mass spectrometry (ID-GC/MS) measurements made at the National Metrology Institute of Australia.

- SRM 3280 Multivitamin/Multielement Tablets ${ }^{123,124}$

SRM 3280 is intended to be used in validating analytical methods for the determination of vitamins, carotenoids, and elements in dietary supplement tablets. The SRM is provided as whole tablets because some of the vitamins are encapsulated to provide stability, and grinding would 
compromise this coating. Value assignments were based on the combined measurements from several different RMPs at NIST and the U.S. Department of Agriculture (USDA), results from an ILS organized by the Grocery Manufacturers Association Food Industry Analytical Chemists Committee (GMA FIACC), and an ILS organized by the European Committee for Standardization (CEN).

\subsection{Value Transfer from an Existing Standard Reference Material or Primary Standard Value assignment for individual measurands can be based on the calibration of a NIST-characterized RMP using a closely matched existing SRM. This is appropriate when preparing a new issue of an existing CRM; however, the value for each measurand must be individually determined regardless of how similar the new material is to the old.}

\section{- Gas NTRMs ${ }^{22,23}$}

A gas NTRM can be developed for any pollutant, concentration, and balance gas combination for which a PS, SRM, and measurement procedure exist. Batches of NTRM gas cylinders are produced by qualified specialty gas providers. The producer monitors the entire batch of cylinders for homogeneity and stability and, if they deem the batch fit-for-purpose, provides NIST with the data. NIST reviews the data and selects a subset of the batch for analysis at NIST. If the data are considered fit-for-purpose, NIST value-assigns the batch.

- SRM 1761 a Low Alloy Steel ${ }^{125}$

This and related SRMs are intended for the evaluation and calibration of instrumental methods of analysis used by the ferrous metals industry. The measurement precision provided by modern $\mathrm{X}$-ray fluorescence (XRF) wavelength dispersive spectrometers enables transfer of elemental composition values from an existing SRM to a new version of similar composition. Transfer is accomplished using calibration standards and enough samples of original and renewal materials to demonstrate the homogeneity of each. Matrix corrections are insignificant when the base matrix mass fractions differ by no more than a few percent and the measurand mass fractions agree within about $10 \%$ or are low enough to guarantee that a linear calibration model adequately represents the response. Calibration of the XRF spectrometer and measurement of SRM 1761 samples ensure that:

$\bigcirc$ the measurement procedure is under control,

$\circ$ the linear calibration model performs as required, and

- all SRMs of the chosen type correlate well in the available mass fraction ranges.

The SRM 1761a certified values are traceable to the PSs and calibrations used to certify the values delivered by SRM 1761.

\subsection{Operationally Defined Measurands}

ISO 17034:2016:3.7 describes an operationally defined measurand as a "measurand that is defined by reference to a documented and widely accepted measurement procedure to which only results obtained by the same procedure can be compared." For these measurands, certification is based on results from the appropriate and validated use of the procedure. Measurement results are obtained by NIST or a collaborator with practical experience using the procedure. The reporting requirements for the collaborators are specified in the certification plan with their reports containing enough detail to permit NIST to evaluate the fitness of the measurements.

Since the "true value" for an operationally defined measurand is the result provided by the procedure, the measurement uncertainty associated with the result combines the procedure's reproducibility imprecision, uncertainty in instrumental bias corrections, and material heterogeneity and instability. Certified values are metrologically traceable to the measurement procedure, which in the case of an operationally defined measurand is the higher-order reference system for that measurand. 
This mode is appropriate when:

- the measurand is uniquely defined by the procedure,

- the procedure is well-documented, typically by a standards development organization or government agency, and

- the procedure is widely known, accepted, and used by the relevant measurement communities.

Selected current examples are:

- SRM 185i Potassium Hydrogen Phthalate pH Standard ${ }^{126,127,128}$

This NIST $\mathrm{pH}$ SRM is intended for use in preparing solutions for calibrating $\mathrm{pH}$ electrodes. The SRM is a high-purity solid that delivers a $\mathrm{pH}$ buffer having a nominal standard $\mathrm{pH}$ value of 4 for $0.05 \mathrm{~mol} / \mathrm{kg}$ solutions prepared according to a specified protocol. Standard $\mathrm{pH}$ values are assigned using the primary direct RMP based on the Harned cell and the conventional value for the single-ion activity coefficient of the chloride ion. The RMP is periodically validated through ILS comparisons with other peer organizations. Material homogeneity is assessed using glass electrode measurements, with previous issues of the SRM analyzed as controls.

- SRM 2812 Rockwell C Scale Hardness - High Range 129,130

SRM 2812 is intended for use in calibration and verification of the performance of Rockwell hardness equipment using the Rockwell C Hardness Scale (HRC), an empirical indentation hardness test that can provide useful information about metallic materials. Each individually certified unit of SRM 2812 is a $64 \mathrm{~mm}$ diameter and $15 \mathrm{~mm}$ thick steel test block. Hardness is measured by indentation tests in accordance with the Rockwell hardness principle defined by the CIPM's Consultative Committee for Mass and Related Quantities (CCM) Working Group on Hardness using the NIST standardizing hardness tester and diamond indenter, as described in the SRM Certificate. The tester is a specially designed measuring instrument using directly loaded dead-weights for applying the required forces, and a laser interferometry displacement sensor for measuring the depth of indentation. The geometry of the NIST standardizing diamond indenter was verified using a stylus instrument.

- SRM 2092 Low- Energy, SRM 2096 High-Energy, and SRM 2098 Super-High-Energy V-Notch Specimens (NIST-Verification, 8-mm Striker) ${ }^{131,132,133,134,135}$

These SRMs are intended for the verification of Charpy impact machines equipped with an 8-mm striker. Each unit consists of a set of alloy steel notched bars needed to perform a single machine verification. The bars have been finished to length, heat-treated, and machined to provide a certified absorbed energy within the ranges (13 J to $20 \mathrm{~J}, 88 \mathrm{~J}$ to $136 \mathrm{~J}, 176 \mathrm{~J}$ to $244 \mathrm{~J}$ ). A Machine Verification Letter and Sticker are provided to the user's facility upon receipt of the fractured specimens and completed questionnaire. This Verification Letter provides the certified values and their associated uncertainties. Depending on which standard is employed, the customer's result is traceable to international standards ASTM E23 - Notched Bar Impact Testing of Metallic Materials or ISO 148-2:2016 Metallic materials - Charpy pendulum impact test - Part 2: Verification of testing machines.

- $\quad$ SRM 1898 Titanium Dioxide Nanomaterial ${ }^{136,137}$

This SRM is intended as a benchmark and investigative tool for evaluation of the potential environmental, health, and safety risks associated with manufactured nanomaterials. A unit consists of $15 \mathrm{~g}$ of mixed anatase and rutile nanocrystalline titanium dioxide $\left(\mathrm{TiO}_{2}\right)$ in the form of a dry agglomerated powder. Single-point and multi-point Brunauer-Emmet-Teller (BET) specific surface area measurements used to establish certified values were obtained using commercial gas sorption instruments at NIST and at three expert collaborating laboratories using a defined method based on a static, volumetric technique traceable to ISO 9277:2010 Determination of the specific surface area of solids by gas adsorption - BET method. The values were validated with results from a 20-participant ILS. 


\subsection{Values Defined by International Convention}

NIST supplies a number of special-purpose international primary measurement standards that are defined by artifact materials value-assigned by international convention rather than measurement. These materials define the zero or span for various isotopic " $\delta$-scales"36 used to unambiguously express small differences in isotope amount or number ratios in the same manner that the International Prototype of the Kilogram ${ }^{138}$ defined the kilogram from 1889 to 2019 . The values delivered by these $\delta$-scale "anchor" materials are intrinsically absolute without uncertainty.

These materials have historically been provided by NIST as RMs. It is intended that by recognizing their special role in defining higher-order reference systems (i.e., the internationally recognized $\delta$ scales), to which metrological traceability for isotope ratio measurement values can be established, they can become SRMs. Examples include:

- Reference Material 8535a Vienna Standard Mean Ocean Water 2 (VSMOW2) and 8537a Standard Light Antarctic Precipitation 2 (SLAP2) ${ }^{139}$

These RMs, known colloquially as VSMOW2 and SLAP2, respectively anchor the zero point and deuterium-depleted end of the $\delta^{2} \mathrm{H}_{\text {VSMOW2-SLAP2 }}$ scale. Each unit of these RMs consists of $20 \mathrm{~mL}$ water in a sealed glass ampoule.

- Reference Material 8544a IAEA-603 (Carbon and Oxygen Isotopes in Carbonate) ${ }^{140}$ This RM defines the Vienna Peedee Belemnite (VPBD) scale for relative differences in carbon (C) and oxygen $(\mathrm{O})$ isotope-number ratios, $R\left({ }^{13} \mathrm{C} /{ }^{12} \mathrm{C}\right.$ and $\left.{ }^{18} \mathrm{O} /{ }^{16} \mathrm{O}\right)$. Each unit of the $\mathrm{RM}$ consists of one bottle containing approximately $0.5 \mathrm{~g}$ of carbonate.

\subsection{Need-Driven Exceptions}

When a certified value is required, but the above approaches are not appropriate, new metrologically defensible modes are defined. Such exceptional circumstances occur when:

- NIST cannot by itself provide the service in a timely manner, for instance when:

- analytical procedures need to be developed and validated in concert with producing the material.

- NIST does not have the specialized expertise, instrumentation, facilities, or regulatory permission needed to establish a critical link in the traceability chain, nor can these be acquired sufficiently quickly.

- Expert collaborators having needed capabilities are available and willing to participate in certification.

- NIST has the expertise to understand the participating organizations' processes and to evaluate the quality of all provided information (as required by ISO 17034:2016:6.2).

- NIST has the expertise to interpret the results.

Under such circumstances, NIST may elect to use the special capabilities or facilities of the expert collaborators to perform laboratory measurements according to NIST-specified designs.

The following illustrate previously encountered challenges and the specialized approaches developed to meet them. Different needs will doubtless arise in the future and require similar inventiveness.

\subsubsection{Analytical Development}

SRM 2855 Additive Elements in Polyethylene is intended for the calibration or evaluation of methods for elemental analysis of polymers. It was developed in collaboration with ASTM International subcommittee D20.70 at a time during which NIST had no RMP for elements in thermoplastics. ${ }^{141,142}$ Suitable analytical procedures had to be developed and validated at the same time as the materials were being evaluated in an ILS. 
- Challenge: At the outset of the project, there were no NIST RMPs for elements in polyethylene.

- Certification procedures: NIST staff developed XRF and ICP-OES measurement procedures for the specific matrix.

- Verification procedure: A commercial set of polyethylene reference materials was used for quality assurance for both NIST RMPs and participant methods. Participants in the ILS reported results using XRF, ICP-OES, and neutron activation analysis.

- Conclusion: The NIST results agreed well with the ILS consensus summaries, validating that the NIST RMPs were fit-for-purpose. Certified values were assigned based on the NIST ICP-OES and XRF results combined with the consensus results from the ILS.

\subsubsection{Expertise}

SRM 3246 Ginkgo biloba (Leaves) ${ }^{53}$ is intended for use in validating analytical methods for the determination of flavonoids, terpene lactones, and toxic elements in botanical matrices.

- Challenge: NIST did not have the taxonomic expertise to identify botanical tissue, hence it did not itself have the equivalent of a higher-order RMP for establishing botanical identity.

- Certification procedure: The leaf material used to prepare SRM 3246 was acquired from a commercial source. The identity and uniform composition of this material was confirmed through morphological examination by a credentialed specialist.

- Verification procedure: DNA analysis of two characteristic chloroplast sequences was used to verify identity. Sequences from the SRM material were compared to multiple Ginkgo biloba voucher specimens (inclusivity panel) and individual voucher specimens or literature sequences of close relatives (exclusivity panel). Confidence in the identification reflects sequence uniformity within the sequences from multiple samples, complete compatibility with the inclusivity panel sequences, and incompatibility with the exclusivity panel sequences. Sequence analysis of multiple samples established genetic uniformity and absence of DNA-containing tissue from common botanical contaminants. (Note: A botanical voucher is "a representative sample of an expertly identified organism that is deposited and stored at a facility from which researchers may later obtain the specimen for examination and further study." ${ }^{143}$ )

- Conclusion: Botanical identity was established with the highest confidence through due diligence in acquisition and verification of the source material. Metrological traceability was provided to the Ginkgo biloba holotype through the established, internationally recognized voucher system. The approach used was considered to be validated for this particular application.

\subsubsection{Instrumentation}

SRM 3121 Gold (Au) Standard Solution ${ }^{144}$ is intended for use as a primary calibration standard for the quantitative determination of gold. Production of this SRM required assessment of the purity of the metallic gold PS used to prepare calibration solutions for the ICP-OES method. Determining impurities in metals requires the use of glow discharge mass spectrometry (GDMS); determining the nonmetallic element content of these materials requires inert gas fusion (IGF) and combustion analysis. These three RMPs are internationally recognized as higher-order procedures for assaying impurities in metal samples.

- Challenge: While NIST staff had the expertise required, NIST did not have at the time the needed GDMS, IGF, or combustion instrumentation.

- Certification procedure: Two sets of GDMS, IGF, and combustion measurements were sourced from expert collaborators. National Research Council Canada (NRCC) and Evans Analytical Group (Liverpool, NY) provided GDMS results. Luvak Laboratories, Inc. (Boylston, MA) provided IGF and combustion analysis results. The purity of the PS was calculated from the combined results at NIST using a NIST-developed, mass-balance purity assessment approach. 
- Verification procedure: Solutions prepared using SRM 3121 were compared to those prepared from archived materials using ICP-OES measurements.

- Conclusion: The certified measurement uncertainty includes components for each of the methods, differences between the two sets of GDMS measurements, an allowance for nondetectable elements, and sample heterogeneity. Metrological traceability is to the SI through the calibration materials used in the analyses. Owing to the verification procedure and a thorough theoretical assessment, the approach was considered validated.

\subsubsection{Facilities and Permission}

SRM 1877 Beryllium Oxide Powder ${ }^{145,146}$ is intended for use in laboratory analysis and health research for the development and validation of analytical methods and instruments used to determine beryllium, supporting the industries that produce high-performance alloys and ceramics. Beryllium oxide $(\mathrm{BeO})$ powder is toxic, and the very fine powder is difficult to confine.

- Challenge: While NIST has the expertise and a higher-order RMP (exact matching ICP-OES) for evaluating the mass fraction of beryllium in solution, it does not have the facilities or required Occupational Health and Safety Administration (OSHA) regulatory approval to work with or package the $\mathrm{BeO}$ powder. ${ }^{147}$

- Certification procedure: Sets of solutions for ICP-OES analysis were prepared by expert collaborators who had the experience, equipment, and regulatory approval needed to work with the powder safely. Each collaborator utilized a unique digestion protocol. After preparation, the solutions were shipped to NIST for analyses.

- Verification procedure: NIST provided the collaborating laboratories with the packaged BeO powder, solution preparation protocols, and quality assurance kits for their analytical balances. Collaborators were asked to confirm the calibration of their balances before they prepared their solutions.

- Conclusion: The collaborating laboratories provided NIST with four independently prepared Be solutions. The NIST RMP results for these gravimetrically prepared solutions agreed within $0.074 \%$, confirming the homogeneity of the material and its fitness for use by the target industries. Given the strong independence in the solution preparations, the results validated the approach for this application.

\subsubsection{Stability and Reassessment}

SRM 972a Vitamin D Metabolites in Frozen Human Serum ${ }^{148,149}$ is intended for use as an accuracy control in the critical evaluation of methods for determining the amount-of-substance concentration of vitamin D metabolites in human serum. The determination of individual vitamin D metabolites required a liquid chromatography-mass spectrometry (LC-MS) procedure and a liquid chromatography-tandem mass spectrometry (LC-MS/MS) RMP at NIST and an LC-MS/MS RMP at the CDC. Several years after the initial certification, the CDC indicated that their refined LC-MS/MS RMP was resulting in $25(\mathrm{OH}) \mathrm{D}_{2}$ values for SRM 972a Level 3 that were slightly outside the certified range.

- Challenge: While NIST staff had the expertise required to perform measurements, staff and instrumentation were not available to run additional SRM 972a samples for stability check and possible reassessment of the $25(\mathrm{OH}) \mathrm{D}_{2}$ certified values in a timely manner. However, both NIST and the CDC had analyzed Levels 2 and 3 of the SRM as control materials during recent vitamin D metabolite measurement campaigns.

- Certification procedure: NIST combined the original certified values for $25(\mathrm{OH}) \mathrm{D}_{2}$ with the NIST and CDC control values. The updated certified values for $25(\mathrm{OH}) \mathrm{D}_{2}$ for Level 2 and Level 3 are consensus means obtained using the Bayesian hierarchical model in the NIST Consensus Builder ${ }^{\mathrm{xxx}}$ on the results from analyses at NIST using ID-LC-MS and ID-LC MS/MS and from CDC using two ID-LC-MS/MS procedures. 
- Verification procedure: All new CDC LC-MS/MS RMP 25(OH) $\mathrm{D}_{2}$ values for Level 3 were calibrated with SRM 2972a 25-Hydroxyvitamin D Calibration Solutions utilizing Level 2 for quality assurance and passing internal QC parameters. All new NIST LC-MS/MS RMP $25(\mathrm{OH}) \mathrm{D}_{2}$ values for Level 3 were calibrated with SRM 2972a 25-Hydroxyvitamin D Calibration Solutions or calibration stocks prepared from neat powder with purity determined in-house at NIST by ${ }^{1} \mathrm{H}-\mathrm{qNMR}$.

- Conclusion: NIST leveraged the most recent knowledge from both in-house measurements and an expert collaborator to update the certified values for $25(\mathrm{OH}) \mathrm{D}_{2}$. The measurement uncertainties include components from the original certified values, the new CDC LC-MS/MS RMP values, and the new NIST LC-MS/MS RMP values. Metrological traceability is to the SI through the measurement results of the calibration materials used.

\subsubsection{User Disruption}

SRM 2806d Medium Test Dust (MTD) in Hydraulic Fluid ${ }^{150}$ is intended for use in the calibration of instrumental response to medium test dust suspended in hydraulic fluid using the ISO 11171:2020 Hydraulic Fluid Power-Calibration of Automatic Particle Counters for Liquids procedure. ${ }^{151}$ The previous version of this material, SRM 2806b, had a particle size distribution other than what the community had become accustomed to. The distributional differences were disruptive to the use of the international standard. ${ }^{152}$

- Challenge: Considerable effort had been expended to establish SI-traceability for the values certified in SRM 2806b Medium Test Dust (MTD) in Hydraulic Fluid. ${ }^{153}$ A different approach was required to provide the user community with replacement materials suited to their established procedures while efficiently delivering values metrologically traceable to the SI.

- Certification procedure: Certified values were derived from 22 ILS datasets provided by 13 international laboratories. Metrological traceability to the SI was achieved by having all participants calibrate their instruments with a material that had been characterized at NIST using SRM $2806 \mathrm{~b}$ as the calibrant.

- Verification procedure: NIST participated in the ILS.

- Conclusion: Total user community buy-in for SRM 2806d was achieved while preserving the metrological advances realized with SRM 2806b. The relevant ISO committee has established a relationship between SI-traceable measurements and the reference system defined by the original SRM 2806. This provides continuity over time and place for measurements made using the ISO 11171 procedure.

\subsection{Recertification}

Some SRMs in areas such as optical properties and gas concentrations may be returned to NIST for recertification and extension of their period of validity. This applies only to SRMs that are periodically produced in small quantities, typically with units that are individually certified.

- The Optical Property SRM series (930, 1930, 2031, and 2930) may be recertified for a two-year period of validity. Recertification involves cleaning as well as redetermining and validating the SRM's originally certified properties.

- Gas cylinder SRMs may be re-certified for a defined period of validity upon request, depending on the pressure of the certified gas remaining in the cylinder.

\subsection{Non-Certified Values}

Any measurement process that provides a defensible fit-for-purpose estimate of a measurand value can be used to assign a non-certified value. 


\section{Acknowledgements}

\subsection{Version}

The authors of the 2020 version of this document thank their NIST colleagues Mary Bedner, Ashley Beasley Green, Ashley Boggs-Russell, Sally S. Bruce, Colleen E. Bryan Sallee, John Kucklick, Elisabeth Mansfield, John Molloy, Karen Murphy, Jacolin Murray, Michael Nelson, Lane Sander, Stacy Schuur, Savelas Rabb, Jason Waters, and Robert L. Watters, Jr. for their help in developing and debugging this document. Cover art courtesy of Lane Sander.

\subsection{Version}

The authors of the 2021 update of this document thank their newly retired colleague John Sieber for his contributions to the development of the 2020 version. The authors thank Chuck Barber, Colleen E. Bryan Sallee, Regina A. Easley, Robert A. Fletcher, Vincent A.D. Hackley, Hugh V. Hayes, Eric L. Kilpatrick, Rebecca A. Kraft, John R. Kucklick, Mark S. Lowenthal, Jacolin Murray, Melissa M. Phillips, Stacy S. Schuur, Nicholas E. Sharp, Aaron A. Urbas, Robert L. Watters, Jr., W. Brent Wilson, Laura J. Wood, and Lee L. Yu for their reviews of and comments on the 2021 revision.

\section{References}

1 May W, Parris R, Beck C, Fassett J, Greenberg R, Guenther F, Kramer G, Wise S, Gills T, Colbert J, Gettings R, MacDonald B. Definitions of Terms and Modes Used at NIST for Value-Assignment of Reference Materials for Chemical Measurements. NIST Special Publication 260-136 (2000). https://www.nist.gov/document-10363

2 Beauchamp CR, Camara JE, Carney J, Choquette SJ, Cole KD, DeRose PC, Duewer DL, Epstein MS, Kline MC, Lippa KA, Lucon E, Phinney KW, Polakoski M, Possolo A, Sharpless KE, Sieber JR, Toman B, Winchester MR, Windover D. Metrological Tools for the Reference Materials and Instruments of the NIST Materials Measurement Laboratory. NIST Special Publication (NIST SP) 260-136, 2020 Edition (2020). https://doi.org/10.6028/NIST.SP.260-136-2020

3 ISO/IEC 17025:2017 General requirements for the competence of testing and calibration laboratories. International Organization for Standardization. Geneva (2017).

4 ISO 17034:2016 General requirements for the competence of reference material producers. International Organization for Standardization. Geneva (2016).

5 JCGM 200:2012 International vocabulary of metrology -Basic and general concepts and associated terms (VIM3). Joint Committee for Guides in Metrology. Sevres, France. (2012).

https://www.bipm.org/en/publications/guides

6 ISO Guide 30:2015 Reference materials - Selected terms and definitions (Guide 30). International Organization for Standardization. Geneva (2015).

7 NIST Quality Manual for Measurement Services NIST-QM-I Version 11. NIST (2019). https://www.nist.gov/document/nistqm-i-v11 controlledandsignedpdf

8 Bruce SS. The NIST Quality System for Measurement Services: A Look at its Past Decade and a Gaze toward its Future. 2013 NCSL International Workshop and Symposium, Nashville, TN. https://tsapps.nist.gov/publication/get pdf.cfm?pub id=913859 Accessed 7/27/2021.

9 Possolo A. Simple Guide for Evaluating and Expressing the Uncertainty of NIST Measurement Results (Simple Guide). NIST Technical Note 1900 (2015). http://doi.org/10.6028/NIST.TN.1900

10 JCGM 100:2008. Evaluation of measurement data - guide to the expression of uncertainty in measurement. Joint Committee for Guides in Metrology (GUM). Joint Committee for Guides in Metrology. Sèvres, France (2008). https://www.bipm.org/en/publications/guides 
11 SRI 6005 Polymerization Stress Tensometer. https://www.nist.gov/sri/standard-referenceinstruments/sri-6005-polymerization-stress-tensometer Accessed 7/27/2021.

12 Chiang MYM, Giuseppetti AAM, Qian J, Dunkers JP, Antonucci JM, Schumacher JE, Gibson S-L. Analyses of a cantilever-beam based instrument for evaluating the development of polymerization stresses, Dental Materials 2011;27(9):899-905. https://doi.org/10.1016/j.dental.2011.05.006

13 SRI 6008 Ozone Standard Reference Photometer. https://www.nist.gov/sri/standard-referenceinstruments/sri-6008-ozone-standard-reference-photometer Accessed 7/27/2021.

14 Specifications, Standard Reference Instrument Series 6008, Ozone Standard Reference Photometer (2017). https://www.nist.gov/document/6008pdf

15 Choquette SJ, Duewer DL, Sharpless KE. NIST Reference Materials: Utility and Future. Ann Rev Anal Chem 2020;13(1):453-474. https://doi.org/10.1146/annurev-anchem-061318-115314

16 Kjeldahl method. https://en.wikipedia.org/wiki/Kjeldahl method. Accessed 7/27/2021.

17 NIST Certificate of Analysis, SRM 1649b Urban Dust (2016). https://www-s.nist.gov/srmors/certificates/1649b.pdf

18 NIST Certificate of Analysis, SRM 141e Acetanilide (2017). https://www-s.nist.gov/srmors/certificates/141e.pdf

19 NIST Certificate of Analysis, SRM 911c Cholesterol (2016). https://www-s.nist.gov/srmors/certificates/911c.pdf

20 NIST Certificate of Analysis, SRM 917c D-Glucose (Dextrose) (2016). https://www-s.nist.gov/srmors/certificates/917c.pdf

21 NIST Certificate of Analysis, SRM 17f D-Sucrose Optical Rotation (2013). https://www-s.nist.gov/srmors/certificates/17f.pdf

22 Guenther FR, Dorko WD, Miller WR, Rhoderick G. The NIST Traceable Reference Material Program for Gas Standards. NIST Special Publication 260-126 (1996). https://doi.org/10.6028/NIST.SP.260-126

23 Dorko WD, Kelley ME, Guenther FR. The NIST Traceable Reference Material Program for Gas Standards. NIST Special Publication 260-126rev2013 (2015). https://doi.org/10.6028/NIST.SP.260-126rev2013

24 Travis JC, Smith MV, Rasberry SD, Kramer GW. Technical Specifications for Certification of Spectrophotometric NTRMs. NIST Special Publication 260-140 (2000).

https://doi.org/10.6028/NIST.SP.260-140

25 IUPAC Compendium of Chemical Terminology (Gold Book), interactive version. International Union of Pure and Applied Chemistry. https://doi.org/10.1351/goldbook

26 Cohen ER, Cvitas T, Frey JG, Holmstrom B, Kuchitsu K, Marquardt R, Mills I, Pavese F, Quack M, Stohner J, Strauss HL, Takami M, Thor AJ. Quantities, Units and Symbols in Physical Chemistry (IUPAC Green Book, 3rd Edition). RSC Publishing, Cambridge, UK (2008). https://iupac.org/wp-content/uploads/2019/05/IUPAC-GB3-2012-2ndPrinting-PDFsearchable.pdf

27 Cohen ER, Giacomo P. Symbols, Units, Nomenclature and Fundamental Constants in Physics. International Union of Pure and Applied Physics (IUPAP), Commission C2 (SUNAMCO). Institute of Physics, London, UK (1987).

https://metrology.wordpress.com/measurement-process-index/iupap-red-book/

28 Analytical Methods Committee. Terminology - the key to understanding analytical science. Part 1: Accuracy, precision and uncertainty. AMC Technical Brief No. 13, September 2003. Available via https://www.rsc.org/Membership/Networking/InterestGroups/Analytical/AMC/TechnicalBriefs.asp 
29 ISO 5725-1 Accuracy (trueness and precision) of measurement methods and results - Part 1: General principles and definitions. International Organization for Standardization. Geneva (1994)

30 Currie LA. Nomenclature in Evaluation of Analytical Methods Including Detection and Quantification Capabilities. Pure \&Appl Chem 1995;67(10):1699-1723.

http://doi.org/10.1351/pac199567101699

31 Izenman AJ. Recent developments in nonparametric density-estimation. J Am Stat Assoc 1991;86(413):205-224. https://doi.org/10.2307/2289732

32 Thompson M, Ellison SLR. Dark uncertainty. Accred Qual Assur 2011;16:483-487. http://doi.org/10.1007/s00769-011-0803-0

33 Koepke A, Lafarge TV, Possolo AM, Toman B. NIST Consensus Builder --- User's Manual (2020). https://consensus.nist.gov/app direct/nicob/NISTConsensusBuilder-UserManual.pdf; https://consensus.nist.gov

34 CIPM Mutual recognition of national measurement standards and of calibration and measurement certificates issued by national metrology institutes. Comite' international des poids et mesures. Paris, 14 October 1999 with Technical Supplement revisions of October 2003 (2003). https://www.bipm.org/en/cipm-mra/cipm-mra-documents

35 World Health Organization. Providing International biologic reference preparations. https://www.who.int/activities/providing-international-biological-reference-preparations Accessed 7/27/2021.

36 Coplen TB. Guidelines and recommended terms for expression of stable-isotope-ratio and gas-ratio measurement results. Rapid Comm Mass Spec 2011;25(17):2538-2560. https://doi.org/10.1002/rcm.5129

37 International Association for Cereal Science and Technology. ICC Standard No. 185 Measurement of Total Dietary Fibre in Cereals, Ingredients and Food Products Using the Rapid Integrated TDF Procedure (RINTDF). 2019. https://icc.or.at/publications/icc-standards/standards-overview/185-standard-method Accessed 7/27/2021.

38 Molloy J, Sieber J. Assessing microscale heterogeneity in batches of reference materials using microbeam XRF. X-Ray Spectrometry 2011;40(4):306-314. https://doi.org/10.1002/xrs.1333

39 Thomas JB, Duewer DL, Kline MC, Sharpless KE. The stability of retinol, $\alpha$-tocopherol, translycopene, and trans- $\beta$-carotene in liquid-frozen and lyophilized serum. Clin Chem Acta 1998;276(1):75-87. https://doi.org/10.1016/S0009-8981(98)00103-X

40 Epstein MS. The reference materials syndrome. Talanta 2010;80:1467-1469. https://doi.org/10.1016/j.talanta.2009.09.028

41 ISO 20387:2018 Biotechnology — Biobanking — General requirements for biobanking. International Organization for Standardization. Geneva (2018).

42 De Bièvre P. "Analyte" is frequently used as synonym for "measurand": is that correct? Accred Qual Assur 2013;18:71-72. https://doi.org/10.1007/s00769-012-0953-8

43 ISO Guide 99:1993 International vocabulary of basic and general terms in metrology (VIM2). International Organization for Standardization. Geneva (1993).

44 Agresti A. An Introduction to Categorical Data Analysis, 3rd Edition, John Wiley \& Sons, Hoboken, NJ (2019)

45 Litmus. https://en.wikipedia.org/wiki/Litmus Accessed 7/27/2021. 
46 Mohs scale of mineral hardness. https://en.wikipedia.org/wiki/Mohs scale of mineral hardness Accessed 7/27/2021.

47 Pendrill L. Quality Assured Measurement. Springer Nature, Cham, Switzerland (2019)

48 NBS, Standard Sample 2106 ISCC-NBS Centroid Color Charts (1964). https://www-s.nist.gov/srmors/certificates/archives/2106.pdf

49 NBS Certificate, SRM 1810 Linerboard Standard for Tape Adhesion Testing (1972). https://www-s.nist.gov/srmors/certificates/archives/1810.pdf

See also: https://www-s.nist.gov/srmors/certificates/1810a.pdf

50 NIST Certificate of Analysis, SRM 1196a Standard Cigarette for Ignition Resistance Testing (2020). https://www-s.nist.gov/srmors/certificates/1196a.pdf

51 Milman BL. Chemical Identification and its Quality Assurance. Springer-Verlag Berlin Heidelberg, Germany, 281 pgs. (2011). https://www.springer.com/us/book/9783642153600

52 Duewer DL. Metrology for Identity and Other Nominal Properties (2015). https://www.slideshare.net/nist-spin/spin-duewer-20141017

53 NIST Certificate of Analysis, SRM 3246 Ginkgo biloba (Leaves) (2019). https://www-s.nist.gov/srmors/certificates/3246.pdf

54 NIST Certificate of Analysis, SRM 1880b Portland Cement (2018). https://www-s.nist.gov/srmors/certificates/1880b.pdf

55 NIST Certificate of Analysis, SRM 3154 Sulfur (S) Standard Solution Lot No. 892205 (2014). https://www-s.nist.gov/srmors/certificates/3154.pdf

56 Guenther FR, Possolo A. Calibration and uncertainty assessment for certified reference gas mixtures. Anal Bioanal Chem 2011;399:489-500. https://doi.org/10.1007/s00216-010-4379-Z

57 Milton MJT, Harris PM, Smith IM, Brown AS, Goody BA. Implementation of a generalized leastsquares method for determining calibration curves from data with general uncertainty structures. Metrologia 2006;43(4):S291-S298. https://doi.org/10.1088/0026-1394/43/4/S17

58 Bartholomew-Biggs M, Butler BP, Forbes AB. Optimization algorithms for generalized distance regression in metrology. In: Ciarlini P, Forbes AB, Pavese F, Richter D (eds) Advanced mathematical and computational tools in metrology IV. Ser Adv Math Appl Sci 2000; 53:21-31. https://doi.org/10.1142/9789812793836 0003

59 Van Huffel S. Total least squares and errors-in-variables modeling. Comp Stat Data Anal 2007;52:1076-1079. https://doi.org/10.1016/j.csda.2007.07.001

60 ASTM E2857 - 11 (Reapproved 2016); Standard Guide for Validating Analytical Methods. ASTM International, West Conshohocken, PA USA (2016).

https://www.astm.org/cgi-bin/resolver.cgi?E2857-11R16

61 Thompson M, Ellison SLR, Wood R. Harmonized guidelines for single-laboratory validation of methods of analysis (IUPAC Technical Report). Pure Appl Chem 2006;74:835-855. https://doi.org/10.1351/pac200274050835

62 Magnusson B, Örnemark U (eds) Eurachem Guide: the fitness for purpose of analytical methods. A Laboratory Guide to Method Validation and Related Topics. Eurachem. (2014).

https://www.eurachem.org/index.php/publications/guides/mv

63 Barrwick V, Ellison SLR, Gjengedal E, Magnusson B, Molinier O, Patriarca M, Sibbesen L, Vanlaethem N, Vercruysse I. Method validation in analytical sciences: discussions on current practice and future challenges. Accred Qual Assur 2017;22:253-263.

https://doi.org/10.1007/s00769-017-1286-4 
64 Alvarez-Prieto M, Jiménez-Chacón J. A fitness for purpose approach to validation and verification of analytical measurements. Accred Qual Assur 2018;23:219-229.

https://doi.org/10.1007/s00769-018-1331-y

65 Bogart J. The Difference Between Primary + Secondary Moisture Measurement. https://blog.kett.com/bid/274676/the-difference-between-primary-secondary-moisture-measurement Posted April 10, 2013. Accessed 7/27/2021.

66 Moody JR, Epstein MS. Definitive Measurement Methods. Spectrochimica Acta B, 1991;46(12):1571-1575. https://doi.org/ https://doi.org/10.1016/0584-8547(91)80160-5

67 Jones GR, Jackson C. The Joint Committee for Traceability in Laboratory Medicine (JCTLM) - its history and operation. Clin Chim Acta. 2016;453:86-94. https://doi.org/10.1016/j.cca.2015.11.016

68 JCTLM database of higher-order reference materials, measurement methods/procedures and services for laboratory medicine and in vitro diagnostics. https://www.bipm.org/jctlm/

69 De Bièvre P, Dybkaer R, Fajgelj A, Hibbert DB. Metrological traceability of measurement results in chemistry: Concepts and implementation. Pure Appl Chem 2011;83(10):1873 1935. https://doi.org/10.1351/PAC-REP-07-09-39

70 Thompson M. A Properly Developed Consensus from a Proficiency Test is, for All Practical Purposes, Interchangeable with a Certified Value for a Matrix Reference Material Derived from an Interlaboratory Comparison. Geostandards Geoanalytical Res 2018;42(1):91-96. https://doi.org/10.1111/ggr.12195

71 Possolo A, Bruce SS, Watters RL Jr. Metrological Traceability Frequently Asked Questions and NIST Policy. NIST Technical Note 2156 (2021). https://doi.org/10.6028/NIST.TN.2156

72 Miller WG, Myers GL. Commutability Still Matters. Clin Chem 2013;59(9):1291-1293. https://doi.org/10.1373/clinchem.2013.208785

73 Miller WG, Schimmel H, Rej R, Greenberg N, Ceriotti F, Burns C, Budd JR, Weykamp C, Delatour V, Nilsson G, MacKenzie F, Panteghini M, Keller T, Camara JE, Zegers I, Vesper HW. IFCC Working Group Recommendations for Assessing Commutability Part 1: General Experimental Design. Clin Chem 2018;64(3):447-454. https://doi.org/10.1373/clinchem.2017.277525

74 FarmSoft. Farm to fork traceability. https://www.farmsoft.com/traceability/farm-to-fork-traceability Accessed 7/27/2021.

75 Moody JR, Greenberg RR, Pratt KW, Rains TC. Recommended Inorganic Chemicals for Calibration. Anal Chem 1988;60(21):1203A-1218A. https://doi.org/10.1021/ac00172a724

76 Staats G. On the role of pure oxide materials for primary calibration and validation in inorganic bulk analysis. Fresenius J Anal Chem 1990;336:132-135. https://doi.org/10.1007/BF00322552

77 Magnusson B, Ellison SLR. Treatment of uncorrected measurement bias in uncertainty estimation for chemical measurements. Anal Bioanal Chem 2008;390:201-213. https://doi.org/10.1007/s00216-007-1693-1

78 Kelly WR, MacDonald BS, Guthrie WF. Gravimetric approach to the standard addition method in instrumental analysis. Anal Chem 2008;80(16):6154-6158. https://doi.org/10.1021/ac702437f

79 Cali JP, LaFleur PD, Reeder DJ, Schaffer R, Uriano GA, Velapoldi RA. National Bureau of Standards Position Paper, A National Understanding for the Development of Reference Materials and Methods for Clinical Chemistry, Boutwell JH, Ed. American Association for Clinical Chemistry, Washington, DC USA (1978). 
80 Cline JP. Use of NIST Standard Reference Materials for Characterization of Instrument Performance; Chapter in Industrial Applications of X-ray Diffraction, ed. by Chung FH and Smith DK. Marcel Dekker, Inc. (2000), pp 903-917.

81 NIST Certificate, SRM 660c Line Position and Line Shape Standard for Powder Diffraction (Lanthanum Hexaboride Powder) (2015). https://www-s.nist.gov/srmors/certificates/660C.pdf

82 Mendenhall MH, Hudson LT, Szabo CI, Henins A, Cline JP. The molybdenum K-shell X-ray emission spectrum. J Phys B: At Mol Opt Phys 2019;52(21):215004.

http://doi.org/10.1088/1361-6455/ab45d6

83 Cheary RW, Coelho AA, Cline JP. Fundamental parameters line profile fitting in laboratory diffractometers. J Res NIST 2004;109:1-25. http://doi.org/10.6028/jres.109.002

84 Cline JP, Mendenhall MH, Black D, Windover D, Henins A. The optics and alignment of the divergent beam laboratory X-ray powder diffractometer and its calibration using NIST Standard Reference Materials. J Res NIST 2015;120:173-222. http://doi.org/10.6028/jres.120.013

85 Burke RW, Mavrodineanu R. Accuracy in Analytical Spectrophotometry, NIST Special Publication 260-81 (1983). https://nvlpubs.nist.gov/nistpubs/Legacy/SP/nbsspecialpublication260-81.pdf

86 NIST Certificate of Analysis, SRM 931h Liquid Absorbance Standard for Ultraviolet and Visible Spectrophotometry (2016). https://www-s.nist.gov/srmors/certificates/931h.pdf

87 Rhoderick GC, Carney J, Guenther FR. NIST Gravimetrically Prepared Atmospheric Level Methane in Dry Air Standards Suite. Anal Chem. 2012;84(8):3802-3810.

https://pubs.acs.org/doi/10.1021/ac300526v

88 Kelley ME, Rhoderick GC, Guenther FR. Development and Verification of Air Balance Gas Primary Standards for the Measurement of Nitrous Oxide at Atmospheric Levels. Anal Chem 2014;86(9):4544-4549. https://pubs.acs.org/doi/10.1021/ac500581b

89 Rhoderick GC, Kelley ME, Miller WR Jr, Norris JE, Carney J, Gameson L, Cecelski CE, Harris KJ, Goodman CA, Srivastava A, Hodges JT. NIST Standards for Measurement, Instrument Calibration, and Quantification of Gaseous Atmospheric Compounds. Anal Chem 2018;90:4711-4718. https://doi.org/10.1021/acs.analchem.7b05310

90 NIST Certificate of Analysis, SRM 1720 Northern Continental Air (Ambient Nominal Amount-ofSubstance Fraction: Carbon Dioxide, Methane, Nitrous Oxide) (2015). https://www-s.nist.gov/srmors/certificates/1720.pdf

91 NIST Certificate of Analysis, SRM 1934 Fluorescent Dyes for Quantitative Flow Cytometry (Visible Spectral Range) (2016). https://www-s.nist.gov/srmors/certificates/1934.pdf

92 Wang L, DeRose PC, Gaigalas AK. Assignment of the Number of Equivalent Reference Fluorophores to Dyed Microspheres. J Res NIST 2016;121:264-281. http://doi.org/10.6028/jres.121.012

93 NIST Certificate of Analysis, SRM 2374 DNA Sequence Library for External RNA Controls (2017). https://www-s.nist.gov/srmors/certificates/2374.pdf

94 Bunk DM, Lowenthal MS. Isotope Dilution Liquid Chromatography-Tandem Mass Spectrometry for Quantitative Amino Acid Analysis. In: Alterman M, Hunziker P (eds) Amino Acid Analysis.

Methods in Molecular Biology (Methods and Protocols), 2012;828:29-38. Humana Press, Totowa, NJ. https://doi.org/10.1007/978-1-61779-445-2_3

95 NIST Certificate of Analysis, SRM 2924 C-Reactive Protein Solution (2017). https:/www-s.nist.gov/srmors/certificates/2924.pdf 
96 NIST Certificate of Analysis, SRM 2973 Vitamin D Metabolites in Frozen Human Serum (High Level) (2017). https://www-s.nist.gov/srmors/certificates/2973.pdf

97 Tai SS-C, Bedner M, Phinney KW. Development of a Candidate Reference Measurement Procedure for the Determination of 25-Hydroxyvitamin $\mathrm{D}_{3}$ and 25-Hydroxyvitamin $\mathrm{D}_{2}$ in Human Serum Using Isotope-Dilution Liquid Chromatography-Tandem Mass Spectrometry.

Anal Chem 2010;82(5):1942-1948. https://doi.org/10.1021/ac9026862

98 Tai SS-C, Nelson MA. Candidate Reference Measurement Procedure for the Determination of (24R),25-Dihydroxyvitamin $\mathrm{D}_{3}$ in Human Serum using Isotope-Dilution Liquid ChromatographyTandem Mass Spectrometry. Anal Chem 2015;87(15):7964-7970.

https://doi.org/10.1021/acs.analchem.5b01861

99 Bosworth CR, Levin G, Robinson-Cohen C, Hoofnagle AN, Ruzinski J, Young B, Schwartz SM, Himmelfarb J, Kestenbaum B, de Boer IH. The serum 24,25-dihydroxyvitamin D concentration, a marker of vitamin D catabolism, is reduced in chronic kidney disease. Kidney international, 2012;82(6):693-700. https://doi.org/10.1038/ki.2012.193

100 NIST Certificate of Analysis, SRM 3037 Arsenous Acid (AsIII) Standard Solution (2018). https://www-s.nist.gov/srmors/certificates/3037.pdf

101 Epstein MS. The Independent Method Concept for Certifying Chemical Composition Reference Materials. Spectrochimica Acta B 1991;46(12):1583-1591. https://doi.org/10.1016/0584-8547(91)80162-V

102 Nelson MA, Waters JF, Toman B, Lang BE, Ruck A, Breitruck K, Obkircher M, Windust A, Lippa KA. A New Realization of SI for Organic Chemical Measurement: NIST PS1 Primary Standard for Quantitative NMR (Benzoic Acid). Anal Chem 2018;90(17):10510-10517. https://doi.org/10.1021/acs.analchem.8b02575

103 NIST Certificate of Analysis, SRM 956d Electrolytes in Frozen Human Serum (2015). https://www-s.nist.gov/srmors/certificates/956d.pdf

104 Tai SS-C, Nelson MA. Candidate Reference Measurement Procedure for the Determination of 24R,25-Dihydroxyvitamin $\mathrm{D}_{3}$ in Human Serum using Isotope-Dilution Liquid ChromatographyTandem Mass Spectrometry. Anal Chem 2015;87(15):7964-7970. https://doi.org/10.1021/acs.analchem.5b01861

105 NIST Certificate, SRM 1003c Glass beads - Particle Size Distribution (2011). https://www-s.nist.gov/srmors/certificates/1003c.pdf

106 NIST Certificate of Analysis, SRM 1974c Organics in Mussel Tissue (Mytilus edulis) (2019). https://www-s.nist.gov/srmors/certificates/1974c.pdf

107 Wise SA, Schantz MM, Hays MJ, Koster BJ, Sharpless KE, Sander LC, Benner BA Jr, Schiller SB. Certification of Polycyclic Aromatic Hydrocarbons in Mussel Tissue and Air Particulate Standard Reference Materials (SRMs). Polycyclic Aromatic Compounds 1996;9:209- 216. https://doi.org/10.1080/10406639608031220

108 Romsos EL, Kline MC, Duewer DL, Toman B, Farkas N. Certification of Standard Reference Material $^{\circledR}$ 2372a Human DNA Quantitation Standard. NIST Special Publication 260-189 (2018). https://doi.org/10.6028/NIST.SP.260-189

109 NIST Certificate of Analysis, SRM 2373 Genomic DNA Standards for HER2 Measurements (2015). https://www-s.nist.gov/srmors/certificates/2373.pdf

110 He HJ, Almeida JL, Lund SP, Steffen CR, Choquette S, Cole KD. Development of NIST standard reference material 2373: Genomic DNA standards for HER2 measurements. Biomol Detect Quantif 2016;8:1-8. https://doi.org/10.1016/j.bdq.2016.02.001 
111 NIST Certificate of Analysis, SRM 2391d PCR-Based DNA Profiling Standard (2019). https://www-s.nist.gov/srmors/certificates/2391d.pdf

112 NIST Certificate of Analysis, SRM 2971 24R,25-Dihydroxyvitamin D Calibration Solution (2019). https://www-s.nist.gov/srmors/certificates/2971.pdf

113 NIST Certificate of Analysis, SRM 3949 Folate Vitamers in Frozen Human Serum (2018). https://www-s.nist.gov/srmors/certificates/3949.pdf

114 Fazili Z, Whitehead R Jr, Paladugula N, Pfeiffer CM. A High-Throughput LC-MS/MS Method Suitable For Population Biomonitoring Measures Five Serum Folate Vitamers and One Oxidation Product. Anal Bioanal Chem 2013;405:4549-4560. https://doi.org/10.1007/s00216-013-6854-9

115 Pfeiffer CM, Fazili Z, McCoy L, Zhang M, Gunter EW. Determination of Folate Vitamers in Human Serum by Stable-Isotope-Dilution Tandem Mass Spectrometry and Comparison with Radioassay and Microbiologic Assay. Clin Chem 2004;50:423-432. https://doi.org/10.1373/clinchem.2003.026955

116 Imbard A, Benoist JF, Blom HJ. Neural tube defects, folic acid and methylation. Int J Environ Res Public Health. 2013;10(9):4352-89. https://doi.org/10.3390/ijerph10094352

117 Thomas JB, Duewer DL, Burdette CQ, Sniegoski LT, Yen JH. Certification of Standard Reference Material $^{\circledR}$ 968f Fat-Soluble Vitamins in Frozen Human Serum. NIST Special Publication 260-188 (2017). https://doi.org/10.6028/NIST.SP.260-188

118 NIST Certificate of Analysis, SRM 1635a Trace Elements in Coal (Subbituminous) (2017). https://www-s.nist.gov/srmors/certificates/1635a.pdf

119 Weidner VR, Mavrodineanu R, Mielenz KD, Velapoldi RA, Eckerle KL, Adams B. Holmium Oxide Solution Wavelength Standard. From 240 to $640 \mathrm{~nm}$ - SRM 2034. NIST Special Publication 260-102 (1986). https://www.nist.gov/document-10445

120 Travis JC, Acosta JC, Andor G, Bastie J, Blattner P, Chunnilall CJ, Crosson SC, Duewer DL, Early EA, Hengstberger F, Kim C-S, Liedquist L, Manoocheri F, Mercader F, Metzdorf J, Mito A, Monardh LAG, Nevask S, Nilsson M, Noël M, Rodriguez AC, Ruíz A, Schirmacher A, Smith MV, Valencia G, van Tonder N, Zwinkels J. Intrinsic Wavelength Standard Absorption Bands in Holmium Oxide Solution for UV/visible Molecular Absorption Spectrophotometry. J Phys Chem Ref Data 2005;34(1):41-56. https://doi.org/10.1063/1.1835331

121 NIST Certificate, SRM 2034 Holmium Oxide Solution Wavelength Standard (240 nm to $650 \mathrm{~nm}$ ) (2016). https://www-s.nist.gov/srmors/certificates/2034.pdf

122 NIST Certificate of Analysis, SRM 2894 Ethanol-Water Solution (Nominal Mass Fraction $0.1 \%$ ) (2013). https://www-s.nist.gov/srmors/certificates/2894.pdf

123 NIST Certificate of Analysis, •SRM 3280 Multivitamin/Multielement Tablets (2019). https://www-s.nist.gov/srmors/certificates/3280.pdf

124 Sander LC, Sharpless KE, Wise SA, Nelson BC, Phinney KW, Porter BJ, Rimmer CA, Thomas JB, Wood LJ, Yen JH, Duewer DL, Atkinson R, Chen P, Goldschmidt R, Wolf WR, Ho I-P, Betz JM. Certification of Vitamins and Carotenoids in SRM 3280 Multivitamin/Multielement Tablets. Anal Chem, 2011;83(1):99-108. https://doi.org/10.1021/ac101953u

125 NIST Certificate of Analysis, SRM 1761a Low Alloy Steel (In Cooperation with ASTM International) (2008). https://www-s.nist.gov/srmors/certificates/1761a.pdf

NIST Certificate of Analysis, SRM 1762a Low Alloy Steel (2015). https://www-s.nist.gov/srmors/certificates/1762a.pdf

$126 \mathrm{Wu}$ YC, Koch WP, Durst WA. Standardization of pH Measurements (1988 Edition). NIST Special Publication 260-53 (1988). https://doi.org/10.6028/NBS.SP.260-53e1988 
127 Buck RP, Rondinini S, Covington AK, Baucke FGK, Brett CMA, Camoes MF, Milton MJT, Mussini T, Naumann R, Pratt KW, Spitzer P, Wilson GS. Measurement of pH. Definition, standards, and procedures (IUPAC Recommendations 2002). Pure Appl Chem 2002;74(11):2169-2200. https://doi.org/10.1351/pac200274112169

128 NIST Certificate of Analysis, SRM 185i Potassium Hydrogen Phthalate pH Standard (2013). https://www-s.nist.gov/srmors/certificates/185i.pdf

129 NIST Certificate, SRM 2812 Rockwell C Scale Hardness - High Range (2013). https://www-s.nist.gov/srmors/certificates/2812.pdf

130 ASTM E18 - 19; Standard Test Methods for Rockwell Hardness and Rockwell Superficial Hardness of Metallic Materials. ASTM International, West Conshohocken, PA USA (2019). http://www.astm.org/cgi-bin/resolver.cgi?E18

131 NIST Certificate, SRM 2092 Low-Energy Charpy V-Notch Specimens (NIST-Verification, 8-mm Striker) (2019). https://www-s.nist.gov/srmors/certificates/2092.pdf

132 NIST Certificate, SRM 2096 High-Energy Charpy V-Notch Specimens (NIST-Verification, 8 mm Striker) (2018). https://www-s.nist.gov/srmors/certificates/2096.pdf

133 NIST Certificate, SRM 2098 Super-High-Energy Charpy V-Notch Specimens (NIST-Verification, $8 \mathrm{~mm}$ Striker) (2019). https://www-s.nist.gov/srmors/certificates/2098.pdf

134 ASTM E23 - 18. Standard Test Methods for Notched Bar Impact Testing of Metallic Materials. ASTM International, West Conshohocken, PA USA (2018). http://www.astm.org/cgi-bin/resolver.cgi?E23

135 ISO 148-2:2016 Metallic Materials - Charpy Pendulum Impact Test - Part 2: Verification of Testing Machines. International Organization for Standardization. Geneva (2016).

136 NIST Certificate of Analysis, SRM 1898 Titanium Dioxide Nanomaterial (2012). https://www-s.nist.gov/srmors/certificates/1898.pdf

137 ISO 9277:2010 Determination of the Specific Surface Area of Solids by Gas Adsorption - BET Method. International Organization for Standardization. Geneva (2010).

138 International Prototype of the Kilogram. https://en.wikipedia.org/wiki/International Prototype of the Kilogram Accessed 727/2021.

139 International Atomic Energy Agency, Reference Sheet for VSMOW2 and SLAP2 International Measurement Standards. IAEA, Vienna, pp. 1-8 (Rev 1 dated 2017-07-11).

https://nucleus.iaea.org/sites/ReferenceMaterials/Shared\%20Documents/ReferenceMaterials/StableIs otopes/VSMOW2/VSMOW2_SLAP2.pdf Accessed 7/27/2021.

140 Assonov S, Groening M, Fajgelj A, Hélie JF, Hillaire-Marcel C. Preparation and characterisation of IAEA-603, a new primary reference material aimed at the VPDB scale realisation for $\delta^{13} \mathrm{C}$ and $\delta^{18} \mathrm{O}$ determination. Rapid Commun Mass Spectrom. 2020;34(20):e8867.

https://doi.org/10.1002/rcm.8867

141 NIST Certificate of Analysis, SRM 2855 Additive Elements in Polyethylene (2019). https://www-s.nist.gov/srmors/certificates/2855.pdf

142 Sieber JR. Interlaboratory Study to Establish Precision Statements for ASTM D6247 Standard Test Method for Determination of Elemental Content of Polyolefins by X-ray Fluorescence Spectrometry, Research Report D10-1251. ASTM International, West Conshohocken, PA USA (2010). https://www.astm.org/DATABASE.CART/RESEARCH_REPORTS/RR-D20-1251.htm Accessed 7/27/2021. 
143 Culley TM. Why vouchers matter in botanical research. Appl Plant Sci. 2013;1(11):apps. 1300076. https://doi.org/10.3732/apps.1300076

144 NIST Certificate of Analysis, SRM 3121 Gold (Au) Standard Solution, Lot No. 170531 (2018). https://www-s.nist.gov/srmors/certificates/3121.pdf

145 NIST Certificate of Analysis, SRM 1877 Beryllium Oxide Powder (2008). https://www-s.nist.gov/srmors/certificates/1877.pdf

146 Winchester MR, Turk GC, Butler TA, Oatts TJ, Coleman C, Nadratowski D, Sud R, Hoover MD, Stefanisk AB. Certification of Beryllium Mass Fraction in SRM 1877 Beryllium Oxide Powder Using High-Performance Inductively Coupled Plasma Optical Emission Spectrometry with Exact Matching. Anal Chem 2009;81:2208-2217. https://doi.org/10.1021/ac802251n

147 OSHA. Beryllium. https:/www.osha.gov/SLTC/beryllium/ Accessed 727/2021.

148 NIST Certificate of Analysis, SRM 972a Vitamin D Metabolites in Frozen Human Serum (2017). https://www-s.nist.gov/srmors/certificates/972a.pdf

149 Phinney KW, Tai SS, Bedner M, Camara JE, Chia RRC, Sander LC, Sharpless KE, Wise SA, Yen JH, Schleicher RL, Chaudhary-Webb M, Maw KL, Rahmani Y, Betz JM, Merkel J, Sempos CT, Coates PM, Durazo-Arvizu RA, Sarafin K, Brooks SPJ. Development of an Improved Standard Reference Material for Vitamin D Metabolites in Human Serum. Anal Chem 2017;89(9):4907-4913. https://doi.org/10.1021/acs.analchem.6b05168

150 NIST Certificate of Analysis, SRM 2806d Medium Test Dust (MTD) in Hydraulic Fluid (2021). https://www-s.nist.gov/srmors/certificates/2806d.pdf

151 ISO 11171:2020; Hydraulic Fluid Power-Calibration of Automatic Particle Counters for Liquids; International Organization for Standardization: Geneva, Switzerland (2020)

https:/www.iso.org/obp/ui/\#iso:std:iso:11171:ed-4:v1:en Accessed 7/27/2021.

152 Schmitigal J. Effect of Transitioning from Standard Reference Material 2806a to Standard Reference Material 2806b for Light Obscuration Particle Countering. US Army Tank Automotive Research, Development, and Engineering Center Technical Report 27809 (2016). https://apps.dtic.mil/sti/pdfs/AD1010793.pdf Accessed 7/27/2021.

153 Fletcher R, Ritchie N, Bright D, Filliben J, Guthrie W. Certification of New Standard Reference Material 2806b Medium Test Dust in Hydraulic Fluid. J Res NIST 2016;121:476-497. https://doi.org/10.6028/jres.121.025 